\title{
Sur la fixation des variables dans une distribution par
}

\author{
S. EOJASIF:WIC'/z (Kraków)
}

Dans un article antérieur [5] j'ai étudié les notions de valeur et de limite en un point pour les distributions d'une variable. Le présent article a pour but l'étude du cas de plusieures variables et surtout l'étude de la notion de fixation des variables (les définitions ont étés signalées dans [4]).

Nous avons montré (dans [5]) comment la notion de valeur se ramène dans le cas d'une variable à celle de différentielle d'ordre supérieur au sens de Denjoy (cf. [1]). Nous établissons ici des théorèmes analogues dans le cas de plusieures variables et pour la notion de fixation ( $\$ 4$ ); toutefois, les démonstrations sont plus difficiles. Elles sont basées sur certains lemmes sur le prolongement des fonctions continues ayant une dérivée d'ordre supérieur nulle $(\S 2)$; on prouve ces lemmes en s'appuyant sur un théorème de $\mathrm{H}$. König (cf. [2]) relatif aux fonctions en question.

Les théorèmes sur les distributions admettant une valeur partout (la propriété d'être déterminée par ses valeurs, l'identification à une fonction) s'étendent au cas de la fixation des variables (§5), où les distributions qui sont des fonctions par rapport à un certain ensemble de variables (selon une idée de L. Schwartz [8]) jouent le rôle de fonctions localement sommables.

Dans la définition de la valeur (ou de la fixation des variables) on pent remplacer la limite

$$
\lim _{\lambda \rightarrow 0+} T\left(x_{0}+\lambda x\right)
$$

par la limite

$$
\lim T\left(\boldsymbol{x}_{0}+\boldsymbol{A} \boldsymbol{x}+\mathbf{s}\right),
$$

où la matrice $\boldsymbol{A}$ et le vecteur $\boldsymbol{s}$ tendent vers zéro de façon que $|\boldsymbol{A}|^{n}=$ $O(\operatorname{det} \boldsymbol{A})$ et $\left.|\boldsymbol{s}|=O(|\boldsymbol{A}|)^{1}\right)$. En général on obtient des conditions plus fortes que celle de l'existence de la valeur et variant selon la manière dont $\boldsymbol{A}$

1) Il est à noter que.'le théorème de Z. Zieleźny sur la constance de la limite (1) dans le cas d'une variable (cf. [9]) est faux pour la limite (1) dans le cas de plusieures variables, tandis qu'il reste vrai pour la limite (2). 
et $\boldsymbol{s}$ convergent vers zéro ( $\$ 6$ ). Dans un cas (où la matrice $\boldsymbol{A}$ reste diagonale) on obtient la notion de valeur directionnelle, pour laquelle on établit des théorèmes plus forts sur la relation entre l'existence de la valeur et la possibilité de fixer des variables (\$7).

On a enfin un théorème sur la régularité de la fixation des variables (§8) donnant une condition nécessaire et suffisante qui consiste en une représentation de la distribution sous forme d'une somme de dérivées des mesures (cf. [7], tome I, p. 91, théorème 27); la démonstration est basée sur un lemme spécial sur le prolongement d'un système de mesures.

Sommaire

§1. Introduction.
1.1-1.3. Notations et terminologie. . . . . . . . . . . . . . . 3

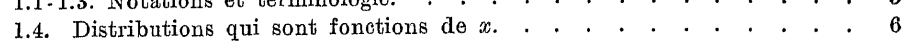

§2. Théorème de H. König et lemines sur le prolongemont.

2.1. Théorème de H. König. . . . . . . . . . . . . . . . . 7 2.2. Un théorème sur les distributions pour lesquelles $T T=0 . \quad$. . . 8

2.3. Une opération de prolongement. . . . . . . . . . . . . . 10

2.4. Lemmes sur le prolongement des fonctions du type $\dot{\mathcal{K}}_{p, q} .+. \quad . \quad 11$

2.5. Un lemme sur le prolongement d'un système de mesures. . . . . 13

§ 3. Définitions. . . . . . . . . . . . . . . . . . . . . . . . 15

$\S 4$. Conditions nécessaires et suffisantes.

4.1-4.2. Condition nécessaire et suffisante avec primitive. . . . . . . . 16

4.3. Fixation dans la primitive et dans la dérivéo. . . . . . . . . . 22

4.4. Relation entre l'existence de la limite et la possibilité de fixer des variables. 23

4.5. Autre forme de la définition de la fixation. . . . . . . . . . 25

4.6. Cas d'une mesure et d'une fonction. . . . . . . . . . . . . . 26

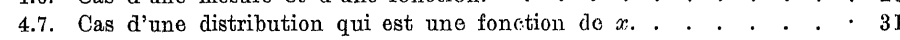

§5. Détermination d'une distribution par ses valeurs.

5.1. Un lemme. . . . . . . . . . . . . . . . . . . . . . 34

5.2. Cas de la valeur. . . . . . . . . . . . . . . . . . . 35

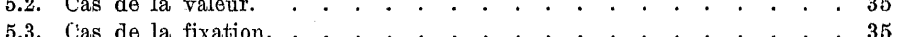

§6. Autres espèces de la convergence dans les définitions du $\$ 3$.

6.1. I.a limite $\operatorname{lin} T\left(\boldsymbol{x}_{0}+\boldsymbol{A} \boldsymbol{x}+\boldsymbol{s}, \boldsymbol{y}\right)$ où $|\boldsymbol{A}|^{m}=O(\operatorname{det} \boldsymbol{A})$ ot $|\boldsymbol{s}|=O(|\boldsymbol{A}|) . \quad 37$

6.2. Onission de la condition $|s|=O(|A|)$. . . . . . . . . . . . 39

6.3. Orrission de la condition $|\boldsymbol{A}|^{m}=O(\operatorname{det} A)$. . . . . . . . . . . 41

6.4. Valeur directionnelle. . . . . . . . . . . . . . . . . . 44

6.5. Condition nécessaire et suffisante avec primitivo pour la valeur dirocitionnelle. . . . . . . . . . . . . . . . . . . . . . 48

$\S 7$. Valeur, fixation et valeur de section.

7.1. Relation on un point. . . . . . . . . . . . . . . . . . 49

7.2. Relation intégrale pour la valeur. . . . . . . . . . . . . 50

7.3. Relation intégrale pour la valeur directionnelle. . . . . . . . 52

7.4. Cas du produit tensoriel. . . . . . . . . . . . . . . . . 54

§8. Ordre de la fixation.

8.1. Une relation entre les bornes des dérivées. . . . . . . . . . 55

8.2. Théorèmes sur l'ordre. . . . . . . . . . . . . . . . . . . . . . . . . . . . . . . 5

8.3. Condition nécessaire et suffisante avec primitive pour l'ordre. . 61

\section{§1. Introduction}

Nous nous occuperons des distributions de plusieurs variables. Pour les définitions et les théorèmes fondamentaux nous renvoyons au livre de L. Schwartz [7]. Les distributions seront considérées localement; nous distinguerons souvent dans une distribution $T(\boldsymbol{x}, \boldsymbol{y})$ les deux ensembles de variables $\boldsymbol{x}=\left(x_{1}, \ldots, x_{m}\right)$ et $\boldsymbol{y}=\left(y_{1}, \ldots, y_{n}\right)$.

Notations et terminologie. 1.1. Soit $\varepsilon$ la droite numérique et $\varepsilon^{m}$ un espace vectoriel de dimension $m$. Nous désignerons les points de $\varepsilon^{m}$ (vecteurs) par des minuscules grasses, leurs coordonées - par des minuscules ordinaires munies d'indices: $\boldsymbol{x}=\left(x_{1}, \ldots, x_{m}\right)$; nous poserons $|\boldsymbol{x}|=\sqrt{x_{1}^{2}+\ldots+x_{m}^{2}} ;$ l'inégalité $\boldsymbol{x} \leqslant \overline{\boldsymbol{x}}$ signifiera $x_{1} \leqslant \bar{x}_{1}, \ldots, x_{m} \leqslant \bar{x}_{m}$. Un intervalle (ouvert, fermé) de $\varepsilon^{m}$ est le produit cartésien d'intervalles bornés (ouverts, fermés) de $\varepsilon$; un intervalle au sens large est le produit cartésien d'intervalles bornés ou non. $|I|$ désignera l'hypervolume d'un intervalle $I$. Nous dirons qu'une suite d'intervalles $I_{v}$ converge régulièrement vers un point $x_{0}$, si $\boldsymbol{x}_{0} \in I_{v}$ et si les longueurs des arêtes tendent vers zéro, leurs rapports mutuels restant bornés.

Les matrices seront désignées par des majuscules grasses: $\boldsymbol{A}=\left[a_{i j}\right]$; nous poserons $|\boldsymbol{A}|=m \max \left|a_{i j}\right| ; \operatorname{det} \boldsymbol{A}=\operatorname{det} a_{i j}$ désignera le déterminant de la matrice $\boldsymbol{A}, \boldsymbol{A} \boldsymbol{x}$ - le produit par un vecteur $\boldsymbol{x}$.

Soit $\mathfrak{Z}$ l'ensemble des entiers et $\chi_{0}$ celui des entiers non négatifs. Ainsi $\Upsilon_{0}^{m}$ est l'ensemble des systèmes $\boldsymbol{p}=\left(p_{1}, \ldots, p_{m}\right)$ d'entiers $\geqslant 0$. Nous poserons $\mathbf{1}=(1, \ldots, 1),|\boldsymbol{p}|=p_{1}+\ldots+p_{m}, \boldsymbol{p} !=p_{1} ! \ldots p_{m} !$,

$$
\left(\begin{array}{l}
\boldsymbol{p} \\
\boldsymbol{s}
\end{array}\right)=\left(\begin{array}{l}
p_{1} \\
s_{1}
\end{array}\right) \ldots\left(\begin{array}{l}
p_{m} \\
s_{m}
\end{array}\right)
$$

et $\boldsymbol{p} \boldsymbol{x}=p_{1} x_{1}+\ldots+p_{m} x_{m}, \boldsymbol{x}^{\boldsymbol{p}}=x_{1}^{p_{1}} \ldots x_{m}^{p_{m}}$, si $\boldsymbol{x} \in \mathcal{E}^{m}$ (on admet $0^{0}=1$ ); $\boldsymbol{p} \leqslant \overline{\boldsymbol{p}}$ signifiera $p_{1} \leqslant \bar{p}_{1}, \ldots, p_{m} \leqslant \bar{p}_{m}$. $D^{\boldsymbol{p}}$ sera le symbole de dérivation partielle:

$$
D^{\boldsymbol{p}}=D_{\boldsymbol{x}}^{\boldsymbol{p}}=\frac{\partial^{p_{1}+\ldots+p_{m}}}{\partial x_{1}^{p_{1}} \ldots \partial x_{m}^{p_{m}}}
$$

On a alors la formule de Newton

et celle de Leibniz

$$
(a+b)^{p}=\sum_{s<p}\left(\begin{array}{l}
p \\
s
\end{array}\right) a^{s} b^{p-s}
$$

$$
D^{\boldsymbol{p}}(\alpha T)=\sum_{\boldsymbol{s} \leqslant \boldsymbol{p}}\left(\begin{array}{l}
\boldsymbol{p} \\
\boldsymbol{s}
\end{array}\right) D^{\boldsymbol{s}} \alpha D^{\boldsymbol{p}-\boldsymbol{s}} T
$$

(où $T$ est une distribution, a une fonction suffisamment régulière). 
1.2. Nous appellerons $D$ (ou $\left.(D)_{x}\right)$ l'ensemble des fonctions $p(\boldsymbol{x})$ de classe $C^{\infty}$ (indéfiniment dérivables) sur $\varepsilon^{m}$ et à support compact Soient $E \subset \mathcal{E}^{m}, k \epsilon \mathcal{R}_{0}, p \epsilon \mathcal{T}_{0}^{m}$; nous désignerons respectivement par $\left(D^{k}\right.$, $\mathscr{D}^{\boldsymbol{p}}, \mathcal{D}_{E},\left(\mathcal{D}_{E}^{k}, \mathcal{D}_{E}^{\boldsymbol{p}}\right)$ les ensembles: des fonctions de classe $C^{k}$ sur $\mathcal{C}^{\mathrm{cm}}$ et à support compact; des fonctions $\varphi$ à support compact ayant des dérivées $D^{s} \varphi, s \leqslant p$, continues dans $C^{(m}$; des fonctions de $D$ (resp. de $D^{k}$, resp. de $\mathfrak{D}^{p}$ ) et à support contenu dans $\boldsymbol{E}$.

Posons

$$
\|\varphi\|_{k}=\max _{|\boldsymbol{p}| \leqslant k} \sup \left|D^{\boldsymbol{p}} \varphi\right| \text { pour } \quad \phi \in\left(T^{k} .\right.
$$

Si $E$ est un ensemble compact de $c^{c m}$, alors $D_{W}^{k}$ est un espace do Banach avec la norme \|\|$_{k}$, dans lequel l'ensemble $\|q\|_{k+1} \leqslant 1$ est précompact. $D_{E}$ est alors un espace $B_{0}$ de Mazur-Orlicz, espace vectoriel métrique complet avec la distance invariante

$$
\varrho(\varphi, \psi)=\sum_{l \in=0}^{\infty} \frac{1}{2^{k}} \frac{\|\varphi-\psi\|_{k}}{1+\|\varphi-\psi\|_{k}} ;
$$

dans cet espace les ensembles $\|\varphi\|_{k} \leqslant 1 / v(k, \nu=1,2, \ldots)$ forment un système fondamental de voisinages de zéro.

Une distribution $T$ définie dans un ouvert $G \subset C^{c m}$ est une fonctionnelle linéaire dans $D_{G}$, continue dans $D_{T}$ pour chaque $E$ compact contenu dans $G$. La valeur de cette forme pour un $\varphi \epsilon D_{G}$, e'est-à-dire le produit scalaire de $T$ par $\varphi$, sera désignée $\operatorname{par}(T, \varphi)$ ou $(T(x), \varphi(x))_{x}$.

Nous dirons qu'une distribution $T$ est d'ordre $\leqslant k$ dans un ensemble $E$, si elle est continue dans $\mathcal{D}_{E}$ selon la norme \|\|$_{k}$; alors $(T, \psi)$ peut être prolongée d'une facon continue sur $\mathscr{D}_{E}^{k}$. Soit $\mathfrak{P}$ un sous-ensemble fini de $\Upsilon_{0}^{m}$; nous dirons que $T$ est d'ordre $\subset \mathfrak{P}$ dans $E$, si $(T, \varphi)$ reste borncé pourvu que $\varphi \in \mathcal{D}_{E}$ et $\left|D^{\boldsymbol{p}} \varphi\right| \leqslant 1$ pour $\left.\boldsymbol{p} \epsilon \mathfrak{P}^{2}\right) ; T$ sera dite d'ordre $\subset \mathfrak{P}$ localement dans un ouvert $G$, si elle est d'ordre $\subset P$ dans tout compact $t$ contenu dans $G$. Soit $G$ un ouvert et $E$ un compact contenu dans $G$; chaque distribution définie dans $G$ est d'ordre fini dans $E$; si une suite $T$, (ou un filtre à base dénombrable) de distributions converge dans $G$ (il s'agit de la convergence simple de $\left(T_{v}, \varphi\right)$ dans $\left.T_{A}\right)$ alors il existe une distribution $T$ et un $k \in \Upsilon_{0}$ tels que $\left(T_{,}, \varphi\right) \rightarrow(T, \varphi)$ dans $D_{G}$, la convergence śtant uniforme pour $\varphi \in \mathcal{D}_{E},\|\varphi\|_{k} \leqslant 1$; enfin, si $\left\{T_{\imath}\right\}_{6 I}$ est une famille de distributions bornée dans $G$ (c'est-à-dire $(T, \varphi)$ est bornée par rapport à l pour chaque $\left.\varphi \epsilon \mathcal{D}_{G}\right)$, alors il existe un $k \epsilon \mathcal{X}_{0}$ et une constante $M$ tels que $\left|\left(T_{t}, \varphi\right)\right| \leqslant$ $\leqslant M$ pour $\iota \in I, \varphi \in \mathcal{D},\|\varphi\|_{k} \leqslant 1$.

2) Si $\Re$ est l'ensemble $|\boldsymbol{p}| \leqslant k$, ceci signifie que $T$ est d'ordre $\leqslant k$
1.3. Si une distribution est une mesure $\mu$, on a $(\mu, \varphi)=\int \varphi d \mu$. Nous désignerons par $\mu(E)$ la valeur de $\mu$ pour un ensemble borélien $E$ et par $|\mu|$ la variation totale de $\mu$. La densité de $\mu$ en un point $x_{0}$ est la limite $d u$ quotient $\mu(I) /|I|$ lorsque $I \rightarrow x_{0}$ régulièrement; elle existe presque partout. Si une mesure $\mu$ est absolument continue, on l'identifie à une fonction localement sommable qui est égale presque partout à la densité de $\mu$. On dit qu'une suite $\left\{\mu_{\nu}\right\}$ (ou un filtre à base dénombrable) de mesures converge faiblement dans $G$, si $\left(\mu_{v}, \varphi\right)$ converge pour tout $\varphi \epsilon D_{G}^{0}$; alors la limite est une mesure dans $G$. Dans le cas des mesures $\geqslant 0$ la convergence au sens des distributions entraine la convergence faible.

Dans une distribution $T$ on peut faire une substitution régulière ${ }^{3}$ ) $\boldsymbol{x}=\boldsymbol{x}(\boldsymbol{u})$; on a alors

$$
\begin{aligned}
& (T(\boldsymbol{x}), \varphi(\boldsymbol{x}))_{\boldsymbol{x}}=\left(T(\boldsymbol{x}(\boldsymbol{u})), \varphi(\boldsymbol{x}(\boldsymbol{u}))\left|\operatorname{det} \frac{\partial x_{i}}{\partial u_{j}}\right|\right)_{\boldsymbol{u}}, \\
& (T(\boldsymbol{x}(\boldsymbol{u})), \psi(\boldsymbol{u}))_{\boldsymbol{u}}=\left(T(\boldsymbol{x}), \psi(\boldsymbol{u}(\boldsymbol{x})) \mid \operatorname{det} \frac{\partial u_{j}}{\partial x_{i}}\right)_{\boldsymbol{x}} .
\end{aligned}
$$

Une substitution linéaire $\overline{\boldsymbol{x}}=\boldsymbol{A} \boldsymbol{x}+\boldsymbol{c}$ conduit d'une mesure $\mu$ à une mesure $\vec{\mu}$ telle que $\vec{\mu}(\bar{E})=|\operatorname{det} \boldsymbol{A}| \mu(E)$. Une substitution régulière conserve l'ordre d'une distribution ainsi que la convergence d'une suite (ou d'un filtre) et la propriété d'une famille de distributions d'être bornée. Une substitution est associative: si $\boldsymbol{x}=\boldsymbol{x}(\boldsymbol{u}), \boldsymbol{u}=\boldsymbol{u}(\boldsymbol{z})$ sont deux substitutions régulières et si $S(\boldsymbol{u})=T(\boldsymbol{x}(\boldsymbol{u})), \boldsymbol{x}(\boldsymbol{z})=\boldsymbol{x}(\boldsymbol{u}(\boldsymbol{z}))$, alors $S(\boldsymbol{u}(\boldsymbol{z}))=$ $=T(\boldsymbol{x}(\boldsymbol{z}))$.

Nous désignerons souvent par $T(\boldsymbol{x}) S(\boldsymbol{y})$ le produit tensoriel $T(\boldsymbol{x}) \times S(\boldsymbol{y})$ et nous écrirons $S(\boldsymbol{y})$ au lieu de $(1)_{\boldsymbol{x}} \times S(\boldsymbol{y})$.

Soit $T(\boldsymbol{x}, \boldsymbol{y})$ une distribution définie dans un ouvert $G \subset\left(\mathcal{E}^{m+n}\right)_{\boldsymbol{x}, \boldsymbol{y}}$ et soit $\varphi \epsilon(D))_{x}$. Nous désignerons par $(T(\boldsymbol{x}, \boldsymbol{y}), \varphi(x))_{\boldsymbol{x}}$ la distribution $S(\boldsymbol{y})$ définie par la formule

$$
(S(\boldsymbol{y}), \psi(\boldsymbol{y}))_{\boldsymbol{y}}=(T(\boldsymbol{x}, \boldsymbol{y}), \psi(\boldsymbol{x}) \psi(\boldsymbol{y})) ;
$$

cette distribution est définie dans l'ensemble des $\boldsymbol{y}$ pour lesquels (support de $\varphi\} \times\{\boldsymbol{y}\} \subset G$. Dans le cas d'une fonction $f(\boldsymbol{x}, \boldsymbol{y})$ on obtient une fonction

$$
y_{1}(\boldsymbol{y})=(f(\boldsymbol{x}, \boldsymbol{y}), \psi(\boldsymbol{x}))_{\boldsymbol{x}}=\int f(\boldsymbol{x}, \boldsymbol{y}) \varphi(\boldsymbol{x}) d \boldsymbol{x},
$$

3) biunivoque, de classe $O^{\infty}$ (ou de classe $O^{k}$ pourvu que $T$ soit d'ordre $\leqslant k$ ) dont le jacobien ne s'annule en aucun point. 
et dans le cas d'une mesure $\mu$ - une mesure $\sigma(\boldsymbol{y})=(\mu(\boldsymbol{x}, \boldsymbol{y}), \varphi(\boldsymbol{x}))_{\boldsymbol{x}}$ pour laquelle

$$
\sigma(E)=\int_{\left(\varepsilon^{m}\right)_{\boldsymbol{x}} \times E} p(\boldsymbol{x}) d \mu(\boldsymbol{x}, \boldsymbol{y}),
$$

lorsque $\{$ support de $\varphi\} \times E \subset G$.

1.4. Distributions qui sont fonctions de $\left.x^{4}\right)$. Soit $E$ un ensemble (mesurable) de $\left(\mathcal{E}^{m}\right)_{x}$ et supposons qu'à tout $x \in E$ (sauf sur un ensemble de mesure nulle) il corresponde une distribution $S_{x}$ définie dans un ouvert $\Omega \subset\left(\mathcal{C}^{n}\right)_{\boldsymbol{y}}$. Nous dirons que la fonction distributionnelle $S_{\boldsymbol{x}}$ est sommabte dans $E \times \Omega$, lorsque

$1^{\circ}\left(S_{x}, \psi\right)$ est une fonction mesurable (de $\left.x\right)$ pour tout $\psi \epsilon\left(D_{\Omega}\right)_{y}$

$2^{\circ}$ il existe un $k \epsilon \chi_{0}$ et une fonction $g(x)$ sommable dans $X$ tels qu'on ait

$$
\left|\left(S_{\boldsymbol{x}}, \psi\right)\right| \leqslant g(x) \quad \text { pour } \quad \boldsymbol{x} \in \mathbb{D}, \psi \in \mathcal{D}_{\Omega} \quad \text { et } \quad\|\psi\|_{k} \leqslant 1 .
$$

Soit $G$ un ouvert quelconque de $\left(\mathcal{E}^{m+n}\right)_{x, y}$; désignons par $\Omega_{x}$ l'ensemble des $\boldsymbol{y}$ pour lesquels $(\boldsymbol{x}, \boldsymbol{y}) \in G$. Supposons qu'à chaque $\boldsymbol{x}$ (pour lequel $\Omega_{x} \neq 0$ ) il corresponde une distribution $S_{x}$ définie dans $\Omega_{x}$. Nous dirons que la fonction distributionnelle $S_{x}$ est continue au point $x_{0}$, si lim $S_{x_{0}}=S_{x_{0}}$

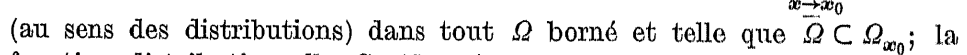
fonction distributionnelle $S_{x}$ (donnée presque partout) sera dite localement sommable dans $G$ lorsqu'elle est sommable dans $P \times Q$, pour chaque couple d'intervalles ouverts $P, Q$ tels que $\overline{P \times Q} \subset G$.

Une fonction distributionnelle continue (en tout point $x$ ) est localement sommable. Si $S_{x}$ est une fonction distributionnelle localement sommable, il en est de même de $S_{x} g(x)$ pourvu que la fonction $g(\boldsymbol{x})$ soit localement bornée (et mesurable). L'intégrale étendue à un ensemble mesurable et borné $E$,

$$
S=\int_{E} S_{x} d x
$$

donnée par la formule $(S, \psi)=\int_{t}\left(S_{\boldsymbol{x}}, \psi\right)_{\boldsymbol{y}} d \boldsymbol{x}$, est définie dans l'ensemble ouvert des $y$ tels que $\left.\bar{E} \times\{y\} \subset C^{\mathbb{E}} G^{5}\right)$.

4) Selon une idée de L. Schwartz (cf. [8]).

5) Dans tous les deux cas au lieu des conditions (1.4.1) il suffit do supposer $\left(S_{x}, \psi\right)$ sommable pour tout $\psi \in \mathcal{D}_{\Omega}$ (on le montre en considérant la fonctionnelle $A(\psi)=\int\left|\left(S_{\boldsymbol{x}}, \psi\right)\right| d \boldsymbol{x}$ qui est finie et semi-continue inférieurement).
Chaque fonction distributionnelle $S_{\boldsymbol{x}}$ localement sommable dans $G$ définit une distribution $T(\boldsymbol{x}, \boldsymbol{y})$ dans $G$ au moyen de la formule

$$
(T, \varphi(\boldsymbol{x}, \boldsymbol{y}))_{\boldsymbol{x}, \boldsymbol{y}}=\int\left(S_{\boldsymbol{x}}, \varphi(\boldsymbol{x}, \boldsymbol{y})\right)_{\boldsymbol{y}} d \boldsymbol{x} .
$$

Nous écrivons alors $T(\boldsymbol{x}, \boldsymbol{y})=S_{x}(\boldsymbol{y})$ et nous disons que la distribution $T$ est une fonction de $x$. La représentation (1.4.2) est d'ailleurs unique: si $T=0$, alors $S_{x}=0$ pour presque tous les $\boldsymbol{x}$.

Considérons le cas où $S_{x}=\sigma_{x}$ est une mesure; pour que la fonction distributionnelle $S_{x}$ soit localement sommable il suffit que $\left|\sigma_{x}\right|(Q)$ soit une fonction sommable dans $P$, pour chaque couple d'intervalles $P, Q$ tels que $\overline{P \times Q} \subset G$ (cette condition est aussi nécessaire dans le cas où $\left.\sigma_{x} \geqslant 0\right)$. Alors $\sigma=\int_{E} \sigma_{x} d x$ est une mesure et on a

$$
\sigma\left(F^{\prime}\right)=\int_{E} \sigma_{\boldsymbol{x}}\left(F^{\prime}\right) d \boldsymbol{x}
$$

pourvu que $E, F$ soient bornés et $\overline{E \times F} \subset G$; pareillement $\mu(\boldsymbol{x}, \boldsymbol{y})=\sigma_{\boldsymbol{x}}(\boldsymbol{y})$ est une mesure et on a (pourvu que $A$ soit borné et $\bar{A} \subset G$ )

$$
\mu(A)=\int \sigma_{\boldsymbol{x}}\left(A_{\boldsymbol{x}}\right) d \boldsymbol{x}
$$

où $A_{\boldsymbol{x}}$ est l'ensemble des $\boldsymbol{y}$ tels que $(\boldsymbol{x}, \boldsymbol{y}) \in A$.

Si $f(\boldsymbol{x}, \boldsymbol{y})$ est une fonction localement sommable dans $G$ et si $S_{\boldsymbol{x}}=f(\boldsymbol{x}, \boldsymbol{y})$ (presque partout en $\left.\boldsymbol{x}\right)$, alors $S_{\boldsymbol{x}}$ est une fonction distributionnelle localement sommable dans $G$ qui définit la distribution $T(\boldsymbol{x}, \boldsymbol{y})=S_{\boldsymbol{x}}(\boldsymbol{y})$ égale à $f(\boldsymbol{x}, \boldsymbol{y})$ dans $G$.

\section{2. Théorème de H. König et lemmes sur le prolongement}

2.1. Théorème de H. König. Nous dirons qu'une fonction $f(x)$ est du type $\chi_{\boldsymbol{p}}$ dans un ensemble $E \subset \mathcal{C}^{m}$, si elle est continue dans $E$ et si

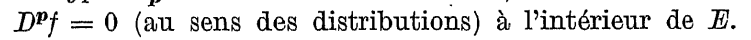

THÉoRìme. Chacune des conditions suivantes est nécessaire et suffisante pour qu'une fonction $f(\boldsymbol{x})$ continue dans un intervalle au sens large $P \subset C^{m}$ soit du type $\mathcal{K}_{\boldsymbol{p}}$ dans $P$ :

$1^{0}$ On a

$$
\Delta_{\boldsymbol{h}}^{\boldsymbol{p}} f(\boldsymbol{x})=\sum_{\boldsymbol{s} \leq \boldsymbol{p}}(-1)^{|\boldsymbol{p}-\boldsymbol{s}|}\left(\begin{array}{l}
\boldsymbol{p} \\
\boldsymbol{s}
\end{array}\right) f\left(x_{1}+s_{1} h_{1}, \ldots, x_{m}+s_{m} h_{m}\right)=0,
$$

lorsque $\boldsymbol{x} \in \boldsymbol{P}$ et $\left(x_{1}+p_{1} h_{1}, \ldots, x_{m}+p_{m} h_{m}\right) \in P$.

$2^{\circ}$ La fonction $f(x)$ est de la forme

$$
f(\boldsymbol{x})=\sum_{i \mid 1}^{m} \sum_{0 \leqslant j<p_{i}} a_{i \nu}\left(x_{1}, \ldots, x_{i-1}, x_{i+1}, \ldots, x_{m}\right) x_{i}^{v}
$$




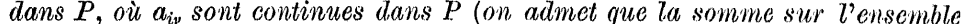
vide $\left(\sum_{0 \leq 0<0}\right)$ est égale à zéro).

Ce théorème a été démontré par $\mathbf{H}$. König dans [2] (pour $f$ et $a_{i_{4}}$ sommables; dans le cas ci-dessus la démonstration est analogne, elle est même un peu plus simple).

2.2. Un théorème sur les distributions pour lesquelles $D^{x} T=0$. Il existe un développement analogue à (2.1.2) pour chaque distribution $T$ telle que $D^{p} T=0$. Notons que les $a_{i p}$ ne sont pas déterminés univoquement, ce qui rend l'usage de ce développement incommode. Nous allons démontrer un théorème qui montre comment on peut faire dépendre les $a_{i v}$ de $T$.

THÉoRÈme. Soit $P_{0}=\left(a_{1}, b_{1}\right) \times \ldots \times\left(a_{m}, b_{m}\right)$ et $\boldsymbol{p} \epsilon \tau_{0}^{m}$. Pour chaque $\left.\boldsymbol{r} \epsilon \Upsilon_{0}^{m}, \mathbf{0}<\boldsymbol{r} \leqslant \mathbf{1}^{6}\right)$ désignons par $\mathfrak{P}_{\boldsymbol{r}}$ l'ensemble des $\mathbf{s} \epsilon \Upsilon_{0}^{m}$ tels que $s_{i}=0$ si $r_{i}=0$ et $0 \leqslant s_{i}<p_{i}$ si $r_{i}=1$; soit $i_{1}<\ldots<i_{|v|}$ la suite complète des $i$ pour lesquels $r_{i}=1$. Il existe un système de fonctions de classe $C^{\infty}$

$$
\alpha_{\boldsymbol{r}, \boldsymbol{s}}\left(x_{i_{1}}, \ldots, x_{i_{|\boldsymbol{r}|}}\right), \quad \text { où } \quad \mathbf{0}<\boldsymbol{r} \leqslant \mathbf{1}, \mathbf{s} \in \mathfrak{P}_{\boldsymbol{r}},
$$

support de $a_{\boldsymbol{r}, \boldsymbol{s}} \subset\left(a_{i_{1}}, b_{i_{\mathbf{1}}}\right) \times \ldots \times\left(a_{i_{|\boldsymbol{r}|}}, b_{i_{|\boldsymbol{r}|} \mid}\right)$, tel que chaque distribution $T$, pour laquelle $D^{p} T=0$ dans un intervalle au sens large $P \supset P_{0}$, soit de la forme

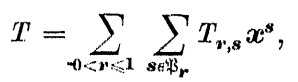

oì

$$
T_{\boldsymbol{r}, \mathbf{s}}=\left(T, \alpha_{\boldsymbol{r}, \mathbf{s}}\right)_{x_{i_{1}}, \ldots, x_{i|\boldsymbol{r}|}}
$$

ne dépend pas de $x_{i_{I}}, \ldots, x_{i_{|\boldsymbol{r}|}}$. Ainsi on peut prendre dans le développement (2.1.2):

$$
a_{i v}=\sum_{\substack{r_{1}=\ldots=r_{i-1}=0 \\ r_{i}=1, s_{i}=\nu}} T_{r, \mathbf{s}} x_{i+1}^{S_{i+1}} \ldots x_{m}^{S_{m}} .
$$

Démonstration. Soit $(a, b)$ un intervalle et $p$ un entier positit. On peut choisir des fonctions $\alpha_{0}, \ldots, a_{p-1} \in D_{(a, b)}$ de telle facon qu'on ait

$$
\left(t^{\prime}, \alpha_{\mu}(t)\right)=-\delta_{\mu^{\nu}} \quad(\mu, \nu=0, \ldots, p-1)
$$

(où $\delta_{\mu \nu}=0$ pourr $v \neq \mu$ et $\delta_{y}=1$ ). En effet, il existe des fonctions $\beta_{0}$, $\ldots, \beta_{p-1} \in \mathcal{D}_{(a, b)}$ telles que $\operatorname{det}\left(t^{\prime \prime}, \beta_{\sigma}\right) \neq 0$ (on pose p. ex. $\beta_{i}(t)=\lambda^{-1} \varrho\left(\left(t-\tau_{i}\right) / \lambda\right)$, où $\varrho$ est une fonction de $D$ pour laquelle $\int \varrho d t \neq 0, a<\tau_{0}<$

\footnotetext{
6) $\mathbf{0}<\boldsymbol{r}$ signifie $\mathbf{0} \leqslant \boldsymbol{r}$ et $\mathbf{0} \neq \boldsymbol{r}$
}

$<\ldots<\tau_{p-1}<b$, et $\lambda$ est suffisamment petit). Il existe alors des nombres $\gamma_{\sigma, \mu}$ tels que

$$
\sum_{\sigma \mid 0}^{p-1}\left(t^{\prime \prime}, \beta_{\sigma}\right) \gamma_{\sigma \mu}=\delta_{\nu \mu},
$$

done on a $\left(t^{\nu}, \alpha_{\mu}\right)=-\delta_{\mu \nu}$ et $\alpha_{\mu} \in \mathcal{D}_{(a, b)}$ pour

$$
a_{\mu}(t)=-\sum_{\sigma \mid 1}^{p-1} \gamma_{\sigma \mu} \beta_{\sigma}(t)
$$

Si maintenant $\varphi \in \mathcal{D}_{(c, d)}$, où $(a, b) \subset(c, d)$, alors la fonction

$$
\psi(t)=\varphi(t)+\sum_{\mu \mid 0}^{p-1}\left(t^{\mu}, \varphi\right) \alpha_{\mu}(t)
$$

est la $p$-ième dérivée d'une fonction de $\mathcal{D}_{(c, d)}$. En effet, on a

$$
\psi(t)=\frac{d^{p} \chi}{d t^{p}}, \quad \text { où } \quad \chi(t)=\int_{-\infty}^{t} \frac{(t-\tau)^{p-1}}{(p-1) !} \psi(\tau) d \tau
$$

est égale, pour $t \geqslant d-\varepsilon$ ( $\varepsilon$ suffisamment petit), a un polynôme de degré $<p$ dont les coefficients s'annulent, car d’après (2.2.4),

$$
\left(t^{\nu}, \psi(t)\right)=\left(t^{\nu}, \varphi(t)\right)-\sum_{\mu \mid 0}^{p-1}\left(t^{\mu}, \varphi\right) \delta_{\nu \mu}=0 ;
$$

on a done $\chi \in \mathcal{D}_{(c, d)}$.

On peut ainsi choisir des fonctions $\alpha_{i \nu} \in \mathcal{D}_{\left(a_{i}, b_{i}\right)}$ de façon que pour chaque $i(i=1, \ldots, m)$ la fonction

$$
\varphi(t)+\sum_{0 \leqslant \nu<p_{i}}\left(t^{\nu}, \varphi(t)\right) \alpha_{i \nu}(t)
$$

soit la $p_{i}$-ième dérivée d'une fonction de $\mathcal{D}_{(c, d)}$ pourvu que $\varphi \epsilon \mathcal{D}_{(c, d)}$ et $\left(a_{i}, b_{i}\right) \subset(c, d)$. Supposons que $P_{0} \subset P=\left(c_{1}, d_{1}\right) \times \ldots \times\left(c_{m}, d_{m}\right)$ et que $p_{i} \in \mathcal{D}_{\left(c_{i}, d_{i}\right)}(i=1, \ldots, m)$. La fonction

$$
\psi\left(x_{i_{1}}, \ldots, x_{m}\right)=\prod_{i \mid \mathrm{i}}^{m}\left[p_{i}\left(x_{i}\right)+\sum_{0 \leqslant \frac{p}{p}<p_{i}}\left(x_{i}^{y}, p_{i}\left(x_{i}\right)\right)_{x_{i}} \alpha_{i v}\left(x_{i}\right)\right]
$$

est la $\boldsymbol{p}$-ième dórivée d'une fonction de $D_{p}$ et, par conséquent, $(T, \psi)=0$. D'autre part, en posant $\alpha_{\boldsymbol{r}, \boldsymbol{s}}\left(x_{i_{1}}, \ldots, x_{i_{|\boldsymbol{r}|} \mid}\right)=-\alpha_{i_{1} s_{1}}\left(x_{i_{1}}\right) \ldots \alpha_{\left.i_{|\boldsymbol{r}|}\right|^{8}|\boldsymbol{v}|}\left(x_{s_{\mid \boldsymbol{r}} \mid}\right)$ on a, d'après (2.2.5), en vertu de (2.2.2),

$$
(T, \psi)=\left(T, \varphi_{1}\left(x_{1}\right) \ldots \varphi_{m}\left(x_{m}\right)\right)-\sum_{0 \leq \boldsymbol{r} \leqslant 1} \sum_{\boldsymbol{s} \in\}_{\boldsymbol{r}}}\left(T_{\boldsymbol{r}, \boldsymbol{s}} \boldsymbol{x}^{\mathbf{s}}, \varphi_{\mathbf{1}}\left(x_{1}\right) \ldots \varphi_{m}\left(x_{m}\right)\right) ;
$$


nous arons done

$$
\left(T, p_{1}\left(x_{1}\right) \ldots \varphi_{m}\left(x_{m}\right)\right)=\left(\sum_{0<r \leqslant 1} \sum_{\boldsymbol{s} \in \sharp^{3} \boldsymbol{r}} T_{\boldsymbol{r}, \boldsymbol{s}} \boldsymbol{x}^{\boldsymbol{s}}, p_{1}\left(x_{1}\right) \ldots \varphi_{m}\left(x_{m}\right)\right),
$$

d'où résulte l'égalité (2.2.1) dans $P$.

Le théorème montre que si $T$ est une mesure, resp. une fonction (localement sommable), resp. une fonction continue etc., il en est de même de $a_{i v}$; en particulier, il contient le théorème de H. König. On peut aussi en déduire les lemmes 1 et 2 du No 2.4 .

2.3. Une opération de prolongement. Soit $p$ un entier positif et $\Delta$ un intervalle fermé, fini ou infini. Appelons $L=L_{l, A}^{p}$ l'opération (linéaire) qui fait correspondre à chaque fonction $f(t)$ définie dans $\Lambda$ son prolongement $\bar{f}=L(f)$ défini dans $\varepsilon$ par les formules

$$
\bar{f}(t)=\left\{\begin{array}{ccc}
f(t) & \text { pour } & t \epsilon \Delta, \\
\sum_{i \mid 0}^{p-1} f\left(t_{i}\right) \prod_{\nu \neq i} \frac{t-t_{v}}{t_{i}-t_{\nu}} & \text { pour } & t \epsilon-\Delta,
\end{array}\right.
$$

où $t_{0}<\ldots<t_{p-1}$ et $t_{0}$ resp. $t_{p-1}$ doit être l'extrémité gauche resp. droite de $\Delta$, pourvu qu'elle soit finie. On vérifie que cette opération possède les propriétés suivantes:

$1^{\prime}$. Si $f$ est continue dans $\Delta$, il en est de même de $\vec{f}$ dans $\mathcal{C}$; si $g(t, y)$ est continue dans $\Delta \times \Omega$, où $\Omega \subset\left(\mathcal{E}^{n}\right)_{y}$ il en est de même de $\bar{g}(t, y)=L_{t}(g)$ dans $\varepsilon \times \Omega$.

$2^{\prime}$. Si $f(t)$ est égale à un polynôme $w(t)$ de degré $<p$ dans $\Delta$, on a $\bar{f}(t)=w(t)$ dans $\varepsilon$.

$3^{\prime}$. Il existe une constante $K=K(p, \Delta)$ telle que si $|f(t)| \leqslant M\left(1+|t|^{p-1}\right)$ dans 4 ; alors $|f(t)| \leqslant K M\left(1+|t|^{p-1}\right)$ dans $\varepsilon$.

Soit maintenant $\boldsymbol{p} \epsilon \mathcal{T}_{0}^{m}, \boldsymbol{p} \geqslant \mathbf{1}$ et $\boldsymbol{P}=\Delta_{1} \times \ldots \times \Delta_{m}$ un intervalle au sens large, fermé. Alors l'opération (linéaire)

$$
L=L_{\mathfrak{x}, P}^{\boldsymbol{p}}=L_{x_{1}, \Delta_{1}}^{p_{1}} \ldots L_{x_{m}, \Delta_{m}}^{p_{m}}
$$

qui fait correspondre à chaque fonction $f(\boldsymbol{x})$ définie dans $P$ son prolongement $\bar{f}=L(f)$ sur $\varepsilon^{m}$, possède les propriétés suivantes:

$1^{\circ}$ Si $f$ est continue dans $P$, il en est de même de $f$ dans $\mathcal{C}^{\prime n} ;$ si $g(x, y)$ est continue dans $P \times \Omega$, où $\Omega \subset\left(\mathcal{E}^{n}\right)_{\boldsymbol{y}}$, il en est de même de $\bar{g}(\boldsymbol{x}, \boldsymbol{y})=$ $=L_{x}(g)$ dans $\left(\varepsilon^{m}\right)_{x} \times \Omega$.

$2^{0}$ Si $f$ est du type $\mathcal{X}_{p}$ dans $P$, il en est de même de $\bar{f}$ dans $C^{c m}$; si $\boldsymbol{g}(\boldsymbol{x}, \boldsymbol{y})$ est du type $\mathcal{K}_{\boldsymbol{p}, \boldsymbol{q}}$ dans $P \times Q$, où $Q \subset\left(\varepsilon^{n}\right)_{\boldsymbol{y}}$ est un intervalle au sens large, il en est de même de $\bar{g}(\boldsymbol{x}, \boldsymbol{y})=L_{\boldsymbol{x}}(g)$ dans $\left(\mathcal{c}^{m}\right)_{\boldsymbol{x}} \times Q$. $3^{\circ}$ Il existe une constante $K=K(\boldsymbol{p}, P)$ telle que si $|f(\boldsymbol{x})| \leqslant M N_{\boldsymbol{p}}(\boldsymbol{x})$ dans $P$, alors $|\bar{f}(\boldsymbol{x})| \leqslant K M N_{\boldsymbol{p}}(\boldsymbol{x})$ dans $\mathcal{C}^{m}$, où

$$
N_{\boldsymbol{p}}(\boldsymbol{x})=\prod_{i \mid 1}^{m}\left(1+\left|x_{i}\right|^{p_{i}-1}\right) \leqslant 2^{m}\left(1+|\boldsymbol{x}|^{|\boldsymbol{p}|-m}\right) .
$$

Les propriétés $1^{\circ}$ et $3^{\circ}$ étant évidentes, nous n'établirons que l'assertion $2^{\circ}$. Il suffit de vérifier que l'opération $L_{x_{i}}^{p_{i}}(i=1, \ldots, m)$ conserve le type $\mathcal{K}_{\boldsymbol{p}, \boldsymbol{\alpha}}$, où nous pouvons admettre $i=1$, sans restreindre la généralité. Supposons donc qu'une fonction $g(\boldsymbol{x}, \boldsymbol{y})$ soit du type $\varkappa_{\boldsymbol{p}, \boldsymbol{\alpha}}$ dans $P \times Q$ et soit $g_{1}(\boldsymbol{x}, \boldsymbol{y})=L_{x_{1}}^{p_{1}}(g)$. Selon le théorème de H. König du No 2.1 nous arons

$$
\begin{aligned}
g(\boldsymbol{x}, \boldsymbol{y})= & \sum_{\nu \mid 0}^{p_{1}-1} a_{1,}\left(x_{2}, \ldots, x_{m}, \boldsymbol{y}\right) x_{1}^{y}+\sum_{i \mid 2}^{m} \sum_{p \mid 0}^{p_{i}-1} a_{i v}\left(\ldots, x_{i-1}, x_{i+1} \ldots, \boldsymbol{y}\right) x_{i}^{y}+ \\
& +\sum_{i, v} b_{i v}\left(\boldsymbol{x}, \ldots, y_{i-1} y_{i+1}, \ldots\right) y_{i}^{v}
\end{aligned}
$$

On a done, d'après la propriété $2^{\prime}$,

$$
\begin{aligned}
g_{1}(\boldsymbol{x}, \boldsymbol{y})= & \sum_{v_{\mid 0}}^{n_{1}-1} a_{1 v}\left(x_{2}, \ldots, x_{m}, \boldsymbol{y}\right) x_{1}^{y}+\sum_{i \mid 2}^{m} \sum_{p \mid 0}^{p_{i^{-1}}} \bar{a}_{i_{v}}\left(\ldots, x_{i-1}, x_{i+1}, \ldots, \boldsymbol{y}\right) x_{i}^{v}+ \\
& +\sum_{i, v} \bar{b}_{i_{v}}\left(\boldsymbol{x}, \ldots, y_{i-1}, y_{i+1}, \ldots\right) y_{i}^{v}
\end{aligned}
$$

en se rappelant que $L_{x_{1}}^{p_{1}}$ est linéaire, où d'après la propriété $1^{\prime}, \bar{a}_{i_{v}}=L_{x_{i}}^{p_{i}}\left(a_{i_{v}}\right)$, $\bar{b}_{i}=L_{x_{i}}^{p_{i}}\left(b_{i_{v}}\right)$ sont continues. Il en résulte que $g_{1}$ est du type $\mathcal{K}_{\boldsymbol{p}, \boldsymbol{q}}$.

2.4. Lemmes sur le prolongement des fonctions du type $\varkappa_{p, q}$. Désignons par $P_{\lambda}$ le cube $\left|x_{i}\right|<\lambda(i=1, \ldots, m)$, et par $P_{\lambda \mu}$ le pavé $\left|x_{i}\right|<\lambda$, $\left|y_{j}\right|<\mu(i=1, \ldots, m ; j=1, \ldots, n)$.

LEM'M 1. Il existe une constante $K=K(\boldsymbol{p}, \boldsymbol{q})$ telle que chaque fonction $g(\boldsymbol{x}, \boldsymbol{y})$ du type $\chi_{\boldsymbol{p}, \boldsymbol{q}}$ dans $\left(\bar{P}_{\lambda}-P_{\mu}\right) \times Q$, où $\mu \leqslant \frac{1}{2} \lambda$ et $Q$ est un intervalle de $\left(\mathcal{E}^{n}\right)_{\boldsymbol{y}}$, possède un prolongement $\bar{g}(\boldsymbol{x}, \boldsymbol{y})$ du type $\mathcal{K}_{\boldsymbol{p}, \boldsymbol{\alpha}}$ dans $\left(-P_{\mu}\right) \times Q$ qui satisfait à l'inégalité

$$
|\bar{g}(\boldsymbol{x}, \boldsymbol{y})| \leqslant K_{\varepsilon} N_{\boldsymbol{p}}\left(\frac{\boldsymbol{x}}{\lambda}\right) \quad \text { dans } \quad\left(-P_{\mu}\right) \times Q
$$

pourvu que $|g(\boldsymbol{x}, \boldsymbol{y})| \leqslant \varepsilon$ dans $\left(\bar{P}_{\lambda}-P_{\mu}\right) \times Q$.

$\mathrm{D}$ émonstration. Le cas général se ramène par homothétie au cas $\lambda=1$, où il suffit d'admettre $\mu=\frac{1}{2}$. Soit $P^{(k)}$ l'intervalle au sens large de $\left(\mathcal{E}^{m}\right)_{x}$ défini par $\left|x_{k+1}\right| \leqslant 1, \ldots,\left|x_{m}\right| \leqslant 1$; on a donc $P^{(0)}=P_{1}, P^{(m)}=$ $=\left(\mathcal{C}^{m}\right)_{x}$. On prolonge $g(\boldsymbol{x}, \boldsymbol{y})$ successivement de $\left(P^{(i-1)}-P_{1 / 2}\right) \times Q$ sur $\left(P^{(i)}-P_{1 / 2}\right) \times Q \quad(i=1, \ldots, m)$, où l'on effectue chacun de ces prolonge- 
ments en faisant deux prolongements au moyen de l'opération $L \mathrm{du} \mathrm{N}^{\circ}$ précédent:

1) de l'intervalle $-1 \leqslant x_{i} \leqslant \frac{1}{2},\left|x_{i+1}\right| \leqslant 1, \ldots,\left|x_{m}\right| \leqslant 1, \boldsymbol{y} \in Q$; sur l'intervalle $-\infty<x_{i} \leqslant \frac{1}{2},\left|x_{i+1}\right| \leqslant 1, \ldots,\left|x_{m}\right| \leqslant 1, \boldsymbol{y} \in Q$

2) de l'intervalle $\frac{1}{2} \leqslant x_{i} \leqslant 1,\left|x_{i+1}\right| \leqslant 1, \ldots,\left|x_{m}\right| \leqslant 1, \boldsymbol{y} \epsilon Q$, sur l'intervalle $\frac{1}{2} \leqslant x_{i}<\infty,\left|x_{i+1}\right| \leqslant 1, \ldots,\left|x_{m}\right| \leqslant 1, \boldsymbol{y} \in Q$.

Ainsi on obtient finalement un prolongement $\bar{g}(\boldsymbol{x}, \boldsymbol{y})$ sur $\left(-P_{1 / 2}\right) \times Q$ qui est du type $\mathcal{K}_{\boldsymbol{p}, \boldsymbol{q}}$ et remplit la condition (2.4.1), en vertu des propriétés $2^{\circ}$ et $3^{\circ}$ de l'opération $L$.

Les propriétés $2^{\circ}$ et $3^{\circ}$ de l'opération $L_{\boldsymbol{x}^{\prime}, y^{\prime}}$ impliquent (il faut faire l'homothétie $\boldsymbol{x}^{\prime}=\lambda \boldsymbol{x}, \boldsymbol{y}^{\prime}=\mu \boldsymbol{y}$ ) le lemme suivant:

Lemute 2. Il existe une constante $K=K(\boldsymbol{p}, \boldsymbol{q})$ telle que chaque fonction $g(\boldsymbol{x}, \boldsymbol{y})$ du type $\mathcal{X}_{\boldsymbol{p}, \boldsymbol{q}}$ dans $\bar{P}_{\lambda, \mu}$ possède un prolongement $\bar{g}(\boldsymbol{x}, \boldsymbol{y}) d u$ type $K_{\boldsymbol{p}, \boldsymbol{q}}$ dans $\mathcal{C}^{\mathrm{m}+n}$ tel que

$$
|\bar{g}(\boldsymbol{x}, \boldsymbol{y})| \leqslant K_{\varepsilon} N_{\boldsymbol{p}, \boldsymbol{\alpha}}\left(\frac{\boldsymbol{x}}{\lambda}, \frac{\boldsymbol{y}}{\mu}\right) \quad \text { dans } \quad \varepsilon^{m+n}
$$

pourvu que $|g(\boldsymbol{x}, \boldsymbol{y})| \leqslant \varepsilon$ dans $\bar{P}_{\lambda, \mu}$.

Nous avons enfin le

LEMNE 3. Il existe une constante $K=K(\boldsymbol{p}, \boldsymbol{q})$ telle que chaque fonction $g(\boldsymbol{x}, \boldsymbol{y})$ du type $\Upsilon_{\boldsymbol{p}, \boldsymbol{q}}$ dans $\bar{P}_{\lambda, \lambda}$ possède un prolongement $\bar{g}(\boldsymbol{x}, \boldsymbol{y}) d u$ type $\mathcal{K}_{\boldsymbol{p}, \boldsymbol{q}}$ dans $\varepsilon^{m+n}$ tel que

$$
\bar{g}(\boldsymbol{x}, \boldsymbol{y}) \leqslant K_{\varepsilon}|\boldsymbol{x}|^{|\boldsymbol{p}|} N_{q}\left(\frac{y}{\lambda}\right) \text { dans } \quad \varepsilon^{m+n}
$$

pourvu que $|g(\boldsymbol{x}, \boldsymbol{y})| \leqslant \varepsilon|\boldsymbol{x}|^{|\boldsymbol{p}|}$ dans $\bar{P}_{\lambda, \lambda}$.

Démonstration. Comme dans les cas précédents, il suffit d'admettre $\lambda=1$. Soit $g(\boldsymbol{x}, \boldsymbol{y})$ une fonction du type $\mathcal{K}_{\boldsymbol{p}, \boldsymbol{q}}$ et telle que $|g(\boldsymbol{x}, \boldsymbol{y})|$ $\leqslant \varepsilon|\boldsymbol{x}|^{|\boldsymbol{p}|}$ dans $\bar{P}_{1,1}$. Alors le prolongement $\overline{\bar{g}}(\boldsymbol{x}, \boldsymbol{y})=L_{\boldsymbol{y}}^{\boldsymbol{q}}(\bar{g})$ sur $\bar{P}_{1} \times\left(\varepsilon^{n}\right)_{\boldsymbol{y}}$ satisfait, selon la propriété $3^{\circ}$, à l'inégalité

(2.4.4) $|\overline{\bar{g}}(\boldsymbol{x}, \boldsymbol{y})| \leqslant K_{1} \varepsilon|x|^{|\boldsymbol{p}|} N_{\boldsymbol{q}}(\boldsymbol{y}) \leqslant n^{|\boldsymbol{p}| / 2} K_{1} \varepsilon N_{\boldsymbol{\alpha}}(\boldsymbol{y}) \quad$ dans $\quad \bar{P}_{1} \times\left(\mathcal{E}^{n}\right)_{\boldsymbol{y}}$

où $K_{1}$ ne dépend que de $\boldsymbol{q}$. Faisons encore une fois le prolongement $\bar{g}(\boldsymbol{x}, \boldsymbol{y})$ $=L_{\boldsymbol{x}}^{\boldsymbol{p}}(\overline{\bar{g}})$ sur $\left(\mathcal{E}^{m}\right)_{\boldsymbol{x}} \times\left(\mathcal{C}^{n}\right)_{\boldsymbol{y}}$. D'après $(2.4 .4)$, en vertu de la propriété $3^{\circ}$, on a $|\bar{g}(\boldsymbol{x}, \boldsymbol{y})| \leqslant K_{2} \varepsilon N_{\boldsymbol{p}}(\boldsymbol{x}) N_{\boldsymbol{q}}(\boldsymbol{y})$ dans $\varepsilon^{m+n}$, où $K_{2}$ ne dépend que de $\boldsymbol{p}$ et $\boldsymbol{q}$; d'autre part, d'après (2.4.4), on a $|\bar{g}(\boldsymbol{x}, \boldsymbol{y})| \leqslant K_{1} \varepsilon|\boldsymbol{x}|^{|\boldsymbol{p}|} N_{\boldsymbol{\alpha}}(\boldsymbol{y})$ dans $\bar{P}_{1} \times\left(\mathcal{E}^{n}\right)_{\boldsymbol{y}}$, d'où, en vertu de $(2.3 .3), \quad|\bar{g}(\boldsymbol{x}, \boldsymbol{y})| \leqslant K \varepsilon|\boldsymbol{x}|^{|\boldsymbol{p}|} N_{\boldsymbol{\alpha}}(\boldsymbol{y})$ dans $\varepsilon^{n+m}$, où $K$ ne dépend que de $\boldsymbol{p}$ et $\boldsymbol{q}$.

2.5. Un lemme sur le prolongement d'un système de mesures. Posons

$$
h_{\sigma}(t)=\left\{\begin{array}{cll}
0 & \text { pour } & t \in 0 \\
\frac{t^{\sigma-1}}{(\sigma-1) !} & \text { pour } & t>0 \quad(\sigma=1,2, \ldots),
\end{array}\right.
$$

$h_{0}(t)=\delta(t)$ (distribution de Dirac) et $H_{\boldsymbol{s}}(\boldsymbol{x})=h_{s_{1}}\left(x_{1}\right) \times \ldots \times h_{s_{m}}\left(x_{m}\right)$; on a alors

$$
D^{s} H_{\boldsymbol{s}}(\boldsymbol{x})=\delta(\boldsymbol{x}) .
$$

Soit $T$ une distribution définie dans un ouvert $\Omega \subset \varepsilon^{m}$ et soit $\alpha \in D_{\Omega}$. Convenons de considérer le produit multiplicatif $T \alpha$ comme défini dans $\mathcal{E}^{m}$ (égal à zéro en dehors du support de $\alpha$ ); en s'appuyant sur les formules de Leibniz et de Newton (cf. $\mathbb{N}^{0} 1.1$ ) on vérifie que

$$
a D^{\boldsymbol{p}} T=\sum_{\boldsymbol{s} \leqslant \boldsymbol{p}}(-1)^{|\mathbf{s}|}\left(\begin{array}{l}
\boldsymbol{p} \\
\boldsymbol{s}
\end{array}\right) D^{\boldsymbol{p}-\boldsymbol{s}}\left(T D^{\boldsymbol{s}} a\right) \quad \text { dans } \quad \varepsilon^{m}
$$

En tenant compte de (2.5.1) nous obtenons done le

LEMLE 1. On a $a D^{\boldsymbol{p}} T=D^{\boldsymbol{p}} \tilde{T}$ dans $\varepsilon^{m}$, où

$$
\tilde{T}=\sum_{\boldsymbol{s} \leqslant \boldsymbol{p}}(-1)^{|\boldsymbol{s}|}\left(\begin{array}{l}
\boldsymbol{p} \\
\boldsymbol{s}
\end{array}\right) H_{\boldsymbol{s}} *\left(T D^{\boldsymbol{s}} \alpha\right) \quad \text { dans } \quad \varepsilon^{m}
$$

si $T$ est une mesure, il en est de même de $\tilde{T}$.

LEMME 2. Soient $\Pi \subset\left(\mathcal{E}^{m+n}\right)_{x, y}$ et $Q \subset\left(\mathcal{C}^{n}\right)_{y}$ des intervalles ouverts. Il existe une constante $K=K(\boldsymbol{p}, \boldsymbol{q}, \Pi, Q)$ telle que chaque mesure $\mu$ définie dans $\Pi$, telle que $|\mu|(\Pi)<\infty$ et $D^{p, \boldsymbol{\alpha}} \mu=0$ dans $\Pi$, possède un prolongement $\bar{\mu}$ sur $\varepsilon^{m+n}$ pour lequel $D^{\boldsymbol{p}, \boldsymbol{\alpha}} \mu=0$ dans $\varepsilon^{m+n}$ et

$$
\bar{\mu}\left(P_{t} \times Q\right) \leqslant K \mu(\Pi)\left(1+|t|^{|\boldsymbol{p}|}\right) .
$$

Démonstration. Selon le théorème $\mathrm{du} \mathrm{N}^{0} 2.2$, on a pour $\mu$ dans $\Pi$ le développement (2.2.1), où $T_{r, \boldsymbol{s}}$ est une mesure $\mu_{\boldsymbol{r}, \boldsymbol{s}}$ définie dans l'intervalle $\Pi_{r, s}$ (qui est une projection de $\Pi$ ). De la manière dont les $\mu_{\boldsymbol{r}, \boldsymbol{s}}=T_{\boldsymbol{r}, \boldsymbol{s}}$ ont été formées il résulte qu'il existe une constante $K_{0}=K_{0}(\boldsymbol{p}, q, \stackrel{\boldsymbol{r}, \boldsymbol{s}}{\Pi})$ telle que

$$
\left|\mu_{r, s}\right|\left(\Pi_{r, s}\right) \leqslant K_{0}|\mu|(\Pi) .
$$

Soit $\mu_{\boldsymbol{r}, \boldsymbol{s}}$ le prolongement de $\mu_{\boldsymbol{r}, \boldsymbol{s}}$ donné $\operatorname{par} \bar{\mu}_{\boldsymbol{r}, \boldsymbol{s}}(E)=\mu_{\boldsymbol{r}, \boldsymbol{s}}\left(E \cap \Pi_{\boldsymbol{r}, \boldsymbol{s}}\right)$; en remplacant $\mu_{r, s}$ par $\bar{\mu}_{r, s}$ dans le développement (2.2.1) nous obtenons un prolongement $\bar{\mu}$ de $\mu$ pour lequel $D_{\boldsymbol{p}, \boldsymbol{q}} \bar{\mu}=0$ dans $\varepsilon^{m+n}$. En s'appuyant 
sur l'inégalité (2.5.4) nous obtenons pour $\mu$ l'inégalité (2.5.3), où $K$ ne dépend que de $\boldsymbol{p}, \boldsymbol{q}, \Pi$ et $Q$.

Lemine 3. Soient $P_{0}, P$ des intervalles ouverts de $\left(\varepsilon^{m}\right)_{x}$ et $Q_{0}, Q$ des intervalles ouverts de $\left(\mathcal{E}^{n}\right)_{y}$ tels que $\bar{P}_{0} \subset P$ et $\bar{Q}_{0} \subset Q$. Soit a une fonction de $\mathcal{D}_{P \times Q}$ telle que $\alpha=1$ dans $P_{0} \times Q_{0}$. Il existe une constante $K=K\left(\boldsymbol{p}, \boldsymbol{q}, P_{0}, P, Q_{0}, Q, \alpha\right)$ telle que l'on puisse faire correspondre à chaque mesure $\mu$ définie dans $P \times Q$ une mesure $\mu$ définie dans $\varepsilon^{m+n}$ telle que $D^{\boldsymbol{p}, \boldsymbol{\alpha}} \bar{\mu}=a D^{\boldsymbol{p}, \boldsymbol{\alpha}} \mu$ dans $\varepsilon^{m+n}, \mu=\bar{\mu}$ dans $P_{0} \times Q_{0}$ et

$$
|\bar{\mu}|\left(P_{t} \times Q\right) \leqslant K|\mu|(P \times Q)\left(1+t^{|\boldsymbol{p}|}\right) .
$$

Démonstration. Soit

$$
\tilde{\mu}=\sum_{\boldsymbol{r} \leqslant \boldsymbol{p}, \boldsymbol{s} \leqslant \boldsymbol{q}}(-1)^{|\boldsymbol{r}|+|\boldsymbol{s}|}\left(\begin{array}{l}
\boldsymbol{p} \\
\boldsymbol{r}
\end{array}\right)\left(\begin{array}{l}
\boldsymbol{q} \\
\boldsymbol{s}
\end{array}\right) H_{\boldsymbol{r}, \boldsymbol{s}} *\left(\mu D^{\boldsymbol{r}, \boldsymbol{s}} \alpha\right) \quad \text { dans } \quad \varepsilon^{m+n},
$$

conformément à (2.5.2). D'après le lemme 1 , on a alors $D^{\boldsymbol{p}, \boldsymbol{\alpha}} \tilde{\mu}=a D^{\boldsymbol{p}, \boldsymbol{\alpha}} \mu$ dans $\varepsilon^{m+n}$, d'où $D^{\boldsymbol{p}, \boldsymbol{\alpha}}(\mu-\tilde{\mu})=0$ dans $P_{0} \times Q_{0}$. Selon le lemme 2 la mesure $\mu-\tilde{\mu}$ possède un prolongement $\sigma$ de $P_{0} \times Q_{0}$ sur $\varepsilon^{m+n}$ tel que $D^{p, \alpha_{\sigma}}=0$ dans $\varepsilon^{m+n}$ et

$$
\text { (2.5.6) } \quad \begin{aligned}
|\sigma|\left(P_{t} \times Q\right) \leqslant K|\mu-\tilde{\mu}| & \left(P_{0}\right. \\
& \left.\times Q_{0}\right)\left(1+t^{|\boldsymbol{p}|}\right) \\
& \leqslant K(|\mu|(P \times Q)+|\tilde{\mu}|(P \times Q))\left(1+t^{|\boldsymbol{p}|}\right) .
\end{aligned}
$$

Si l'on pose $\bar{\mu}=\tilde{\mu}+\dot{\sigma}$ dans $\varepsilon^{m+n}$ on trouve $D^{\boldsymbol{p}, \boldsymbol{\alpha}} \bar{\mu}=\alpha D^{\boldsymbol{p}, \boldsymbol{\alpha}} \mu$ dans $\varepsilon^{m+n}$ et $\bar{\mu}=\mu$ dans $P_{0} \times Q_{0}$. En vertu de (2.5.6), il suffit done de démontrer que $|\tilde{\mu}|\left(P_{t} \times Q\right) \leqslant K_{1}|\mu|(P \times Q)\left(1+t^{|\boldsymbol{p}|}\right)$ (où $K_{1}$ ne dépend que de $\boldsymbol{p}, \boldsymbol{q}$, $\alpha, P, Q)$. Soit $\varphi \in D_{P_{t}} \times Q,|\varphi| \leqslant 1$; il suffit de prouver que

$$
\text { (2.5.7) } \quad|(\tilde{\mu}, \varphi)| \leqslant K_{1}|\mu|(P \times Q)(1+t)^{|\boldsymbol{p}|} \text {. }
$$

Nous arons

$$
(2.5 .8) \quad\left(H_{\boldsymbol{r}, \boldsymbol{s}} * \mu D^{\boldsymbol{r}, \boldsymbol{s}} \alpha, \varphi\right)=\left(H_{\boldsymbol{r}, \boldsymbol{s}}(\boldsymbol{x}, \boldsymbol{y}), \psi_{\boldsymbol{r}, \boldsymbol{s}}(\boldsymbol{x}, \boldsymbol{y})\right),
$$

où $\psi_{\boldsymbol{r}, \boldsymbol{s}}(\boldsymbol{x}, \boldsymbol{y})=\int D^{\boldsymbol{r}, \boldsymbol{s}} \alpha(\boldsymbol{\xi}, \boldsymbol{\eta}) \varphi(\boldsymbol{x}+\xi, \boldsymbol{y}+\boldsymbol{\eta}) d \mu(\boldsymbol{\xi}, \boldsymbol{\eta})$.

Soient $K_{0}$ et $l$ des constantes telles que $\left|D^{\boldsymbol{r}, \boldsymbol{s}} \alpha\right| \leqslant K_{0}$ pour $\boldsymbol{r} \leqslant \boldsymbol{p}$, $\boldsymbol{s} \leqslant q$ et que $P \times Q$ soit dans le cube $\left|x_{i}\right| \leqslant l,\left|y_{j}\right| \leqslant l$. On a alors $\left|\psi^{\boldsymbol{r}, \boldsymbol{s}}(\boldsymbol{x}, \boldsymbol{y})\right|$ $\leqslant K_{0}|\mu|(P \times Q)$ et support de $\psi_{r, s} \subset P_{l_{+t}} \times Q_{1}$, où $Q_{1}$ est le cube $\left|y_{j}\right| \leqslant 2 l$. Nous avons done $\left|\left(H_{\boldsymbol{r}, \boldsymbol{s}}, \psi_{\boldsymbol{r}, \boldsymbol{s}}\right)\right| \leqslant(t+l)^{|\boldsymbol{r}|}(2 l)^{|\boldsymbol{s}|} K_{0}|\mu|(P \times Q)$ selon la définition de $H_{r, s}$ et, d'après $(2.5 .5)$ et (2.5.8), nous obtenons l'inégalité (2.5.7) avec une constante $K_{1}=K_{1}(\boldsymbol{p}, \boldsymbol{q}, \alpha, P, Q)$, ce qui termine la démonstration.

LEMME 4. Soit $\subseteq$ un ensemble fini de $\Upsilon_{0}^{m+n}$ et soient $Q_{0}, Q$ des intervalles ouverts de $\left(\mathcal{E}^{n}\right)_{y}$ tels que $Q_{0} \subset Q$. Il existe une constante $K=K\left(\mathcal{S}, Q_{0}, Q\right)$ telle que chaque système de mesures $\varrho_{\boldsymbol{p}, \boldsymbol{q}},(\boldsymbol{p}, \boldsymbol{q}) \in \mathcal{S}$, définies dans $P_{2 \lambda} \times Q$, satisfaisant à l'égalité $\sum_{\overparen{E}} D^{p, q} \varrho_{\boldsymbol{p}, \boldsymbol{q}}=0$ dans $P_{2 \lambda} \times Q$, possède un prolongement

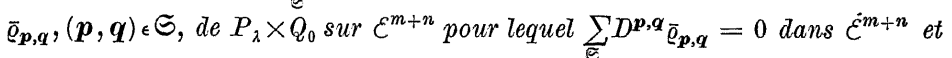

$$
\left|\bar{\varrho}_{\boldsymbol{p}, \boldsymbol{\alpha}}\right|\left(P_{t} \times Q\right) \leqslant K\left|\varrho_{\boldsymbol{p}, \boldsymbol{q}}\right|\left(P_{2 \lambda} \times Q\right)\left(1+\left|\frac{t}{\lambda}\right|^{|\boldsymbol{p}|}\right) \quad \text { pour } \quad t>0 .
$$

Démonstration. Comme dans les démonstrations du № 2.4, il suffit d'admettre $\lambda=1$. Formons pour chaque mesure $\varrho_{\boldsymbol{p}, \boldsymbol{q}}$ son prolongement $\bar{\varrho}_{\boldsymbol{p}, \boldsymbol{q}}$ de $P_{1} \times Q_{0}$ sur $\varepsilon^{m+n}$, selon le lemme 3. On a alors $\left|\bar{\varrho}_{\boldsymbol{p}, \boldsymbol{q}}\right|\left(P_{t} \times Q\right)$ $\leqslant K\left|\varrho_{\boldsymbol{p}, \boldsymbol{\alpha}}\right|\left(P_{2} \times Q\right)\left(1+t^{t \boldsymbol{p} \mid}\right)$, où $K$ ne dépend que de $\gamma, Q_{0}, Q$, et $\sum_{\mathscr{夭}} D^{\boldsymbol{p}, \boldsymbol{q}} \varrho_{\boldsymbol{p}, \boldsymbol{\alpha}}$ $=\sum_{\overparen{S}} \alpha D^{\boldsymbol{p}, \boldsymbol{\alpha}} \varrho_{\boldsymbol{p}, \boldsymbol{\alpha}}=0$ dans $\varepsilon^{m+n}$.

\section{§3. Définitions}

La définition de la valeur en un point dans le cas de plusieurs variables reste la même que dans le cas d'une variable (cf. [4]). On dit qu'une distribution $T$, définie dans un voisinage d'un point $\boldsymbol{x}_{0} \in \mathcal{E}^{m}$, possède la valeur $C$ au point $\boldsymbol{x}_{0}: T\left(\boldsymbol{x}_{0}\right)=C$, si la limite

$$
\lim _{\lambda \rightarrow 0+} T\left(x_{0}+\lambda x\right)=C
$$

existe dans un voisinage de $\boldsymbol{x}_{0}{ }^{7}$ ) et si elle est constante $\left.{ }^{8}\right)$.

Soit $\Omega$ un ouvert de $\left(\mathcal{E}^{n}\right)_{y}$ et $T(\boldsymbol{x}, \boldsymbol{y})$ une distribution définie dans un ouvert de $\left(\mathcal{E}^{m+n}\right)_{x, y}$ qui contient l'ensemble $\left\{x_{0}\right\} \times \Omega$. Nous disons que dans la distribution $T(\boldsymbol{x}, \boldsymbol{y})$ on peut fixer $\boldsymbol{x}=\boldsymbol{x}_{0}$ sur $\Omega$, si la limite

$$
\lim _{\lambda \rightarrow 0+} T\left(\boldsymbol{x}_{0}+\lambda \boldsymbol{x}, \boldsymbol{y}\right)=S(\boldsymbol{y})
$$

existe et ne dépend que de $y$ dans un ouvert contenant $\left.\left\{x_{0}\right\} \times \Omega^{9}\right)$. La distribution $S(\boldsymbol{y})$ définie dans $\Omega$ s'appelle la section de $T$ pour $\boldsymbol{x}=\boldsymbol{x}_{0}$ et $\boldsymbol{y} \epsilon \Omega$. On écrit

$$
S(\boldsymbol{y})=T\left(\boldsymbol{x}_{0}, \boldsymbol{y}\right) .
$$

Soit $T(\boldsymbol{x}, \boldsymbol{y})$ une distribution définie dans $G-\left\{\boldsymbol{x}_{0}\right\} \times \bar{\Omega}$, où $\Omega$ est un ouvert de $\left(\varepsilon^{n}\right)_{\boldsymbol{y}}$ et $G$ un ouvert de $\left(\varepsilon^{m+n}\right)_{\boldsymbol{x}, \boldsymbol{y}}$ contenant $\left\{\boldsymbol{x}_{0}\right\} \times \Omega$. On dit que la distribution $T$ possède une limite sur $\Omega$ pour $\boldsymbol{x} \rightarrow \boldsymbol{x}_{0}$ :

$$
S(\boldsymbol{y})=\lim _{x \rightarrow x_{0}} T(\boldsymbol{x}, \boldsymbol{y}) \quad \text { dans } \quad \Omega,
$$

7) Alors il en est de même dans tout ouvert borné.

8) La limite (3.1) peut existor sans être constante (cf. $\mathrm{N}^{\circ} 6.1$ ).

9) Alors il en est de même dans tout ouvert borné de la forme $\Delta \times \Omega_{0}$ où $\Delta \subset\left(\mathcal{E}^{m}\right)_{x}, \bar{\Omega}_{0} \subset \Omega$. 
si la limite (3.2) existe et ne dépend que de $y$ dans un ouvert de la forme $\left.G_{1}-\left\{x_{0}\right\} \times \Omega^{10}\right)$ où $G_{1}$ est un ouvert contenant $\left\{x_{0}\right\} \times \Omega$.

Dans le cas de la limite en un point $(n=0)$ on exige, comme dans le cas d'une variable (cf. [5]) que la limite (3.1) existe et soit constante dans un voisinage de $x_{0}$ pour $x \neq x_{0}$.

On définit encore la limite suivant un cone ouvert $\Gamma<\left(\mathcal{E}^{m}\right)_{x}$ de sommet $\boldsymbol{x}_{0}, \lim _{\boldsymbol{x} \rightarrow \boldsymbol{x}_{0}, \boldsymbol{x} \in T} T(\boldsymbol{x}, \boldsymbol{y})=S(\boldsymbol{y})$, en exigeant que la limite (3.2) existe et ne dé-

pende que de $\boldsymbol{y}$ dans l'ensemble de la forme $(\Gamma \times \Omega) \cap G$, où $G$ est un ouvert contenant $\left\{x_{0}\right\} \times \Omega$. En particulier $(m=1)$, nous avons les définitions des limites unilatérales $\lim T(\boldsymbol{x}, \boldsymbol{y})$ et $\lim T(\boldsymbol{x}, \boldsymbol{y})$.

On voit que dans le cas d'une fonction $\underset{x \rightarrow x_{0}-}{\boldsymbol{x} \rightarrow \boldsymbol{x}_{0}+}$ usuelles (voir $\mathrm{N}^{\mathrm{s}} 4.6$ et 4.7 pour le cas d'une fonction localement sommable). Elles ont un caractère local; p. ex. si $T_{1}(\boldsymbol{x}, \boldsymbol{y})=T_{2}(\boldsymbol{x}, \boldsymbol{y})$ dans un ouvert contenant $\left\{\boldsymbol{x}_{0}\right\} \times \Omega$, alors les sections $T_{1}\left(x_{0}, \boldsymbol{y}\right)$ et $T_{2}\left(\boldsymbol{x}_{0}, \boldsymbol{y}\right)$ sur $\Omega$ n'existent qu'en même temps et on a $T_{1}\left(\boldsymbol{x}_{0}, \boldsymbol{y}\right)=T_{2}\left(\boldsymbol{x}_{0}, \boldsymbol{y}\right)$ dans $\Omega$; si les sections d'une distribution pour $\boldsymbol{x}=\boldsymbol{x}_{0}$ sur $\Omega_{1}$ et sur $\Omega_{2}$ existent, elles coïncident dans $\Omega_{1} \cap \Omega_{2}$. On vérifie que la section d'une somme est égale à la somme des sections et il en est de même de la valeur, de la limite et dans le cas du produit par une fonction indéfiniment dérivable.

On montre enfin que ces notions sont invariantes pour les substitutions régulières, ce qui permet de définir une substitution régulière dans une distribution $x_{1}=x_{1}\left(u_{1}, \ldots, u_{k}\right), \ldots, x_{m}=x_{m}\left(u_{1}, \ldots, u_{k}\right)$, dans le cas où $k<m$.

\section{\$4. Conditions nécessaires et suffisantes}

Nous allons étendre maintenant les théorèmes de [5] (\$2) qui ont ramené les notions de valeur et de limite d'une distribution d'une variable à celle du $n^{\text {ième }}$ quotient différentiel au sens de Denjoy.

Conditions nécessaires et suffisantes avec primitive. 4.1. Soit $\left\{\lambda_{v}\right\}$ une suite décroissante telle que

$$
\lambda_{v} \rightarrow 0 \quad \text { et } \quad \liminf _{v \rightarrow \infty} \frac{\lambda_{v+1}}{\lambda_{v}}>0 .
$$

On peut donc choisir une constante $\vartheta$ de sorte que $0<\vartheta<1$ et $\lambda_{v+1} / \lambda_{v}>\vartheta$ pour $\nu \geqslant N$. Définissons par récurrence une suite extraite $\left\{\lambda_{a_{v}}\right\}$ de façon que $\alpha_{1}=N$ et que $a_{v+1}$ soit l'indice le plus petit possible $\left.{ }^{10}\right)$ Alors il en est de même dans tout ouvert borné de la forme $\left(\Delta-\left\{x_{0}\right\}\right) \times \Omega_{0}$,
où $\Delta \subset\left(\varepsilon^{m}\right)_{x}, \bar{\Omega}_{0} \subset \Omega$. pour lequel $\alpha_{v}<\alpha_{v+1}$ et $\lambda_{\alpha_{v+1}} / \lambda_{\alpha_{v}} \leqslant \vartheta$. On a alors $\lambda_{\alpha_{v+1}-1} / \lambda_{\alpha_{v}}>\vartheta$ et, comme $\lambda_{\alpha_{p+1}} / \lambda_{\alpha_{y+1}-1} \geqslant \vartheta$, on obtient $\lambda_{\alpha_{p+1}} / \lambda_{\alpha_{y}} \geqslant \vartheta^{2}$. Nous avons donc

$$
0<\vartheta^{2}<\frac{\lambda_{a_{v+1}}}{\lambda_{\alpha_{v}}}<\vartheta<1 .
$$

Désignons par $P_{\lambda}$ le cube $\left|x_{i}\right|<\lambda(i=1, \ldots, m)$. Soit $P=P_{\sigma}$ et soit $Q$ un intervalle ouvert de $\left(\mathcal{E}^{m}\right)_{y}$. Nous avons

Proposition 1. Soit $T$ une distribution définie dans un ouvert contenant l'ensemble $(\bar{P}-\{0\}) \times \bar{Q}$ et supposons qu'on ait dans cet ouvert

$$
T\left(\lambda_{v}, \boldsymbol{x}, \boldsymbol{y}\right) \rightarrow 0 \quad \text { pour } \quad \nu \rightarrow \infty .
$$

$\Pi l$ existe alors un $(\boldsymbol{p}, \boldsymbol{q}) \epsilon \mathcal{T}_{0}^{m+n}$ et une fonction $F(\boldsymbol{x}, \boldsymbol{y})$ continue dans $\bar{P} \times \bar{Q}$ tels que

$$
\text { (1.4.1) } \quad T=D_{\boldsymbol{x}}^{\boldsymbol{p}} D_{\boldsymbol{y}}^{\boldsymbol{q}} F \quad \text { dans } \quad(P-\{0\}) \times Q \text { et } \lim _{\boldsymbol{x} \rightarrow 0} \frac{F(\boldsymbol{x}, \boldsymbol{y})}{|\boldsymbol{x}|^{\mid \boldsymbol{p} !}}=0
$$

uniformément dans $Q$.

Démonstration. Sans restreindre la généralité nous pouvons admettre $P=P_{1}, \lambda_{1}=1$ et (en tenant compte de (4.1.2))

$$
0<\vartheta^{2}<\frac{\lambda_{v+1}}{\lambda_{v}}<\vartheta<1 \quad(v=1,2, \ldots) .
$$

L'ensemble $\left(\bar{P}-P_{\theta^{2} / 2}\right) \times \bar{Q}$ étant contenu dans le domaine de convergence de (4.1.3), il existe un $(\boldsymbol{p}, \boldsymbol{q}) \in \mathcal{T}_{0}^{m+n}$, où $\boldsymbol{p} \geqslant I$, et une suite $\left\{g_{v}(\boldsymbol{x}, \boldsymbol{y})\right\}$ de fonctions continues convergeant uniformément vers 0 dans $\left(\bar{P}-P_{\vartheta^{2} / 2}\right) \times \bar{Q}$, telle que $T\left(\lambda_{v} \boldsymbol{x}, \boldsymbol{y}\right)=D g_{v}(\boldsymbol{x}, \boldsymbol{y})$ dans $\left(P-\bar{P}_{\vartheta^{2} / 2}\right) \times Q$, où $D=D_{x}^{\boldsymbol{p}} D_{\boldsymbol{y}}^{\boldsymbol{q}}$ On a donc $\left|g_{v}(\boldsymbol{x}, \boldsymbol{y})\right| \leqslant \varepsilon_{v}$ dans $\left(\bar{P}-P_{\theta^{2} / 2}\right) \times \bar{Q}$, où $\varepsilon_{v} \rightarrow 0$ en décroissant. En posant $F_{v}(\boldsymbol{x}, \boldsymbol{y})=\lambda_{v}^{|\boldsymbol{p}|} g_{v}\left(\boldsymbol{x} / \lambda_{v}, \boldsymbol{y}\right)$, on obtient

$$
T=D F \text {, }
$$

et

$$
\left|F_{v}(\boldsymbol{x}, \boldsymbol{y})\right| \leqslant \lambda_{v}^{|\boldsymbol{p}|} \varepsilon_{v}
$$

dans l'ensemble

$$
R_{v}=\left(P_{\lambda_{v}}-\bar{P}_{\lambda_{v^{2}} / 2}\right) \times Q \quad(v=1,2, \ldots) .
$$

D'après (4.1.6) la fonction $q_{v}=F_{v+1}-F_{v}$ est du type $\mathcal{K}_{\boldsymbol{p}, \boldsymbol{q}}$ dans $\bar{R}_{\nu} \cap \bar{R}_{v+1}=\left(\bar{P}_{\lambda_{v+1}}-P_{\lambda_{v} \vartheta^{2} / 2}\right) \times Q$, selon (4.1.5) on a $\frac{1}{2} \lambda_{v} \vartheta^{2}<\frac{1}{2} \lambda_{\nu+1}$, donc le lemme $1 \mathrm{du} \mathrm{N}^{0} 2.4$ est applicable. Il en résulte que la fonction $q_{v}$ possède Studia Mathematica T. XVII 
un prolongement $\bar{q}_{v}$ du type $\mathcal{K}_{\boldsymbol{p}, \boldsymbol{q}}$ sur l'adhérence de l'ensemble

\section{(4.1.8)}

$$
S_{v}=\left(P-\bar{P}_{\vartheta} \lambda_{\nu / 2}\right) \times Q,
$$

qui satisfait à l'inégalité

(4.1.9) $\left|q_{\nu}(x, y)\right| \leqslant 2 K \varepsilon_{\nu} \lambda_{\nu}^{|\boldsymbol{p}|} N_{\boldsymbol{p}}\left(\frac{\boldsymbol{x}}{\lambda_{\nu+1}}\right) \leqslant \frac{2^{m+1} K}{\vartheta^{2|\boldsymbol{p}|}} \varepsilon_{\nu}\left|\lambda_{\nu+1}\right|^{m}\left(\lambda_{\nu+1}^{|\boldsymbol{p}|-m}+|\boldsymbol{x}|^{|\boldsymbol{p}|-m}\right)$

dans $S_{,}$, en vertu de (4.1.7), (4.1.5) et (2.3.3). Nous allons définir des fonctions $\vec{F}_{v}$ par récurrence comme il suit. Posons $\bar{F}_{1}=F_{1}$ dans $S_{1}=R_{1}$. Admettons que $\bar{F}_{v}$ soit définie dans $S_{v}$ et que $\bar{F}_{\nu}=F_{p}$ dans $R_{v}$; comme on a alors $F_{v+1}=\bar{F}_{v}+\bar{q}_{v}$ dans $R_{v} \cap R_{v+1}=S_{v} \cap R_{v+1}$, on peut définir $\bar{F}_{v+1}$ dans $S_{v+1}=R_{v+1}+S_{v}$ par les formules

$$
\bar{F}_{v+1}=\left\{\begin{array}{lll}
F_{v+1} & \text { dans } & R_{v+1} \\
\bar{F}_{v}+\bar{q}_{v} & \text { dans } & S_{v} .
\end{array} .\right.
$$

Nous avons ainsi défini la suite $\left\{\bar{F}_{\nu}\right\}$ de fonctions continues et telles que

$$
\text { (4.1.11) } \quad T=D \bar{F}_{v} \quad \text { dans } \quad S_{v} \quad(v=1,2, \ldots)
$$

(en vertu de (4.1.6)). De la seconde formule (4.1.10) on tire $\bar{F}_{\nu+\sigma}-\bar{F}_{\text {, }}$ $=\bar{q}_{v}+\ldots+\bar{q}_{v+\sigma-1}$ dans $S_{v}$ et, par suite, l'inégalité (4.1.9) donne la majoration

$$
\left|\bar{F}_{\nu+\sigma}-\bar{F}_{\nu}\right| \leqslant \bar{K} \varepsilon_{v} \lambda_{\nu}^{m}\left(\lambda_{\nu}^{|\boldsymbol{p}|-m}+|\boldsymbol{x}|^{|\boldsymbol{p}|-m}\right) \quad \text { dans } \quad S_{\nu},
$$

où $\bar{K}$ est une constante indépendante de $v$ et de $x$, car, d'après (4.1.5), on a

$$
\varepsilon_{\nu} \lambda_{\nu+1}^{m}+\ldots+\varepsilon_{\nu+\sigma} \lambda_{\nu+\sigma+1}^{m} \leqslant \varepsilon_{\nu} \lambda_{m}^{\nu} \frac{\vartheta^{m}}{1-\vartheta^{m}} .
$$

On en conclut que la suite $\left\{\bar{F}_{\nu}\right\}$ converge uniformément dans chacun des ensembles $S_{v}$; sa limite $F$ est donc continue et satisfait à l'inégalité

$$
\left|\boldsymbol{F}-\overline{\boldsymbol{F}}_{\nu}\right| \leqslant \bar{K} \varepsilon_{v} \lambda_{v}^{m}\left(\lambda_{\nu}^{|\boldsymbol{p}|-m}+|\boldsymbol{x}|^{|\boldsymbol{p}|-m}\right) \quad \text { dans } \quad \boldsymbol{S}_{v} .
$$

D'après (4.1.11) on a, de plus, $T=D F$ dans $(P-\{0\}) \times Q=\bigcup_{1}^{\infty} S_{\nu}$ (cf. (4.1.8)). Finalement les inégalités (4.1.7) et (4.1.12) donnent (en tenant compte de $(4.1 .10)) \quad\left|F^{\prime}\right| \leqslant(1+2 \bar{K}) \varepsilon_{v} \lambda_{v}^{|p|}$ dans $R_{v}(v=1,2, \ldots)$, ce qui montre que

$$
\lim _{x \rightarrow 0} \frac{F(\boldsymbol{x}, \boldsymbol{y})}{|\boldsymbol{x}|^{|\boldsymbol{p}|}}=0
$$

uniformément dans $Q$. La proposition est ainsi démontrée.
Soit $\delta>0$ et soit $\omega$ un ensemble ouvert sur la sphère $|x|=1$. Dé signons par $\Gamma(\omega, \delta)$ le cône défini par $\boldsymbol{x} /|\boldsymbol{x}| \epsilon \omega, 0<|\boldsymbol{x}|<\delta$.

Propostrion 2. Etant donnée une distribution $T$ définie dans $\Gamma(\omega, \delta) \times \Omega$, où $\delta>0$, soit $\omega$ un ouvert sur la sphère $|x|=1$ et $\Omega$ celui de $\left(\mathcal{C}^{n}\right)_{y}$ et supposons que

$$
T\left(\lambda_{\boldsymbol{v}} \boldsymbol{x}, \boldsymbol{y}\right) \rightarrow 0 \quad \text { pour } \quad \boldsymbol{v} \rightarrow \infty \text { dans } \Gamma(\omega, \delta) \times \Omega ;
$$

ò̀ $\lambda_{v}^{l}$ satisfait à la condition (4.1.1). Si $\bar{\omega}_{0} \subset \omega, \bar{\Omega}_{0} \subset \Omega\left(\omega_{0}, \Omega_{0}\right.$ étant des ouverts bornés) et $0<\delta_{0}<\delta$, alors il existe un $(\boldsymbol{p}, \boldsymbol{q}) \in \mathcal{C}_{0}^{m_{+}+n}$ et une fonction $F$ continue dans $\Gamma\left(\omega_{0}, \delta_{0}\right) \times \Omega_{0}$ tels que

$$
\left\{\begin{array}{l}
T=D_{\boldsymbol{x}}^{\boldsymbol{p}} D_{\boldsymbol{y}}^{\boldsymbol{q}} F \text { dans } \Gamma\left(\omega_{0}, \delta_{0}\right) \times \Omega_{0}, \\
\text { et } \lim _{\boldsymbol{x} \rightarrow 0, \frac{\boldsymbol{x}}{|\boldsymbol{x}|} \in \omega_{0}} \frac{F(\boldsymbol{x}, \boldsymbol{y})}{|\boldsymbol{x}|^{|\boldsymbol{p}|}}=0 \text { uniformément dans } \Omega_{0} .
\end{array}\right.
$$

Démonstration. Soient $\omega_{1}$ et $\Omega_{1}$ des ouverts bornées tels que $\bar{\omega}_{0} \subset \omega_{1}, \bar{\omega}_{1} \subset \omega, \bar{\Omega}_{0} \subset \Omega_{1}, \bar{\Omega}_{1} \subset \Omega$ et soit $\delta_{0}<\delta_{1}<\delta$; il existe alors des fonctions $\alpha(\boldsymbol{u}), \dot{\beta}(\tau), \gamma(y)$ de classe $C^{\infty}$ telles que

$\alpha(\boldsymbol{u})=\left\{\begin{array}{l}1 \\ \text { dans } \omega_{0}, \\ 0 \text { dans }-\omega_{1},\end{array} \quad \beta(\tau)=\left\{\begin{array}{l}1 \text { pour } \tau<\delta_{0}, \\ 0 \text { pour } \tau \geqslant \delta_{1},\end{array} \quad \gamma(\boldsymbol{y})=\left\{\begin{array}{l}1 \text { dans } \Omega_{0}, \\ 0 \text { dans }-\Omega_{1} .\end{array}\right.\right.\right.$

Par conséquent la distribution

$$
S(\boldsymbol{x}, \boldsymbol{y})=\left\{\begin{array}{ccl}
\alpha\left(\begin{array}{c}
\boldsymbol{x} \\
|\boldsymbol{x}|
\end{array}\right) \beta(|\boldsymbol{x}|) \gamma(\boldsymbol{y}) T(\boldsymbol{x}, \boldsymbol{y}) & \text { dans } & \Gamma(\omega, \delta) \times \Omega, \\
0 & \text { dans } & -\left(\Gamma\left(\omega_{1}, \delta_{1}\right) \times \Omega_{1}\right),
\end{array}\right.
$$

est bien définie dans $\left(\varepsilon^{m+n}\right)_{x, y}$ et on a

$$
S=T \quad \text { dans } \quad \Gamma\left(\omega_{0}, \delta_{0}\right) \times \Omega_{0}
$$

et $S\left(\lambda_{y} \boldsymbol{x}, \boldsymbol{y}\right) \rightarrow 0$ pour $\nu \rightarrow \infty$ (dans chaque ouvert borné de $\left(\varepsilon^{m+n}\right)_{x, y}^{r}$ ). En vertu de (4.1.15) il suffit done d'appliquer la proposition 1.

Remarque. Dans les propositions 1 et 2 on peut exiger que l'on ait $\boldsymbol{p} \geqslant \boldsymbol{p}_{0}, \boldsymbol{q} \geqslant \boldsymbol{q}_{\mathbf{0}}\left(\boldsymbol{p}_{0}, \boldsymbol{q}_{\mathbf{0}}\right.$ étant données $)$.

Voici une proposition d'un caractère réciproque:

Propostrion 3. Soit $G$ un ouvert borné de la forme

$$
\text { (4.1.16) } \quad G=\Delta \times \Omega, \text { où soit } \mathbf{0} \epsilon \Delta \text { soit } \Delta=\Gamma(\omega, \delta)
$$

$\left(\Delta \subset\left(\mathcal{E}^{m}\right)_{\boldsymbol{x}}, \Omega \subset\left(\mathcal{E}^{n}\right)_{\boldsymbol{y}}\right)$. Si $T=D_{\boldsymbol{x}}^{\boldsymbol{p}} D_{\boldsymbol{y}}^{\boldsymbol{q}} F$ dans $G$, ò̀ $F$ est une fonction continue dans $G$ et telle que 


$$
\lim _{x \rightarrow 0, x_{\theta \Delta}} \frac{\boldsymbol{F}(\boldsymbol{x}, \boldsymbol{y})}{|\boldsymbol{x}|^{|\boldsymbol{p}|}}=0 \quad \text { uniformément dans } \Omega,
$$

alors $\lim T(\lambda \boldsymbol{x}, \boldsymbol{y})=\mathbf{0}$ dans $G$.

En effet, la relation (4.1.17) donne

$$
\lim _{\lambda \rightarrow 0+} \frac{1}{\lambda^{|\boldsymbol{p}|}} F(\lambda x, \boldsymbol{y})=0
$$

uniformément dans $G$, d'où l'on obtient la conclusion en effectuant la différentiation $D=D_{x}^{p} D_{y}^{q}$.

CoRoLlarRe. Soit $\left\{\lambda_{\nu}\right\}$ une suite décroissante qui satisfait d̀ (4.1.1) et $G$ un ouvert borné de la forme (4.1.16). Les conditions

$$
\lim _{\boldsymbol{y} \rightarrow \infty} T\left(\lambda_{\nu} \boldsymbol{x}, \boldsymbol{y}\right)=0 \quad \text { et } \quad \lim _{\lambda \rightarrow 0+} T(\lambda \boldsymbol{x}, \boldsymbol{y})=0
$$

dans $G$ sont équivalentes.

Dans les cas où

$$
\lim _{\nu \rightarrow \infty} \frac{\lambda_{\nu+1}}{\lambda_{\nu}}=0
$$

(et $\lambda_{v} \rightarrow 0$ en décroissant) la première des conditions (4.1.18) n'entraîne pas la seconde. En effet, posons $\xi_{p}=\sqrt{\lambda_{v} \lambda_{p+1}}$, il vient

$$
\xi_{v} / \lambda_{v} \rightarrow 0 \quad \text { et } \quad \xi_{v-1} / \lambda_{v} \rightarrow \infty
$$

On peut donc choisir $c_{i}>0(i=1,2, \ldots)$ de sorte que .

$$
\mu_{v}=\frac{\sum_{i \mid v}^{\infty} c_{i}}{\lambda_{\nu}} \rightarrow 0 \quad \text { et } \quad \varrho_{v}=\frac{\sum_{i \mid v}^{\infty} c_{i}}{\xi_{v}} \rightarrow \infty .
$$

Considérons la distribution $T=\sum_{i \mid 1}^{\infty} c_{i} \delta\left(x-\xi_{i}\right)$. La distribution

$$
\dot{T}\left(\lambda_{\nu} \boldsymbol{x}, \boldsymbol{y}\right)=\sum_{i \mid 1}^{\infty} \frac{c_{i}}{\lambda_{\nu}} \delta\left(x-\frac{\xi_{i}}{\lambda_{\nu}}\right)
$$

est une mesure positive; en vertu de (4.1.19) la valeur de cette mesure pour l'intervalle $(-1,1)$ est $\mu_{v}$ pourvu que $v$ soit suffisament grand, donc, d'après (4.1.20), $T\left(\lambda_{v} x\right) \rightarrow 0$ dans $(-1,1)$. D'autre part $T$ est une mesure positive dont la densité, si elle existe, est lim $\varrho_{\nu}$; par conséquent, d'après (4.1.20), cette mesure ne peut avoir une densité finie en 0 , ce qui montre (cf. [4], corrollaire $1 \mathrm{du} \mathrm{N}^{0} 3.3$ et théorème du $\mathrm{N}^{0} 4.3$ ) que la limite $\lim T(\lambda x)$ n'existe dans aucun voisinage de 0 (pour $x \neq 0$ ).

$$
\lim _{\lambda \rightarrow 0+}
$$

4.2. Les propositions du No précédent donnent des conditions nécessaires et suffisantes pour la possibilité de fixer des variables et pour l'existence de la valeur ou de la limite d'une distribution. En effet, si l'on pose $T_{0}(\boldsymbol{x}, \boldsymbol{y})=T\left(\boldsymbol{x}_{0}+\boldsymbol{x}, \boldsymbol{y}\right)-S(\boldsymbol{y})$, la condition $T\left(\boldsymbol{x}_{0}+\lambda \boldsymbol{x}, \boldsymbol{y}\right)$ $\rightarrow S(\boldsymbol{y})$ équivaut à la condition $T_{0}(\lambda \boldsymbol{x}, \boldsymbol{y}) \rightarrow 0$ (où $\lambda \rightarrow 0$ ). Remarquons, de plus, que $S=D_{y}^{\boldsymbol{q}} g$ (dans un ouvert arbitraire d'adhérence compacte et contenue dans le domaine de définition de $S$ ) pour un $q \epsilon\urcorner \tau_{0}^{n}$ et une fonction continue $g(\boldsymbol{y})$; par suite

$$
S=D_{x}^{p} D_{y}^{\boldsymbol{q}} \frac{\left(\boldsymbol{x}-x_{0}\right)^{p}}{p !} g(\boldsymbol{y})
$$

Nous avons done les théorèmes suivants:

THÉORł̀ME 1. Soit $\Omega$ un ouvert de $\left(\varepsilon^{n}\right)_{y}$ et $G C\left(\varepsilon^{m+n}\right)_{x, y}$ un ouvert contenant l'ensemble $\left\{\boldsymbol{x}_{0}\right\} \times \Omega$. Supposons qu'une distribution $T$ soit définie dans $G$ (resp. dans $\left.G-\left\{\boldsymbol{x}_{0}\right\} \times \bar{\Omega}\right)$. Pour qu'on puisse fixer $\boldsymbol{x}=\boldsymbol{x}_{0}$ sur $\boldsymbol{\Omega}$ (resp. qu'il existe une limite pour $\boldsymbol{x} \rightarrow \boldsymbol{x}_{0}$ sur $\Omega$ ) il faut et il suffit que pour tout ouvert borné $\Omega_{0}$ tel que $\bar{\Omega}_{0} \subset \Omega$ il existe un $(\boldsymbol{p}, \boldsymbol{q}) \epsilon \mathcal{T}_{0}^{m+n}$, un voisinage $\Delta$ de $\boldsymbol{x}_{0}$, une fonction $F(\boldsymbol{x}, \boldsymbol{y})$ continue dans $\Delta \times \Omega_{0}$ (resp. dans $\left.\left(\Delta-\left\{\boldsymbol{x}_{0}\right\}\right) \times \Omega_{0}\right)$ et une fonction $g(\boldsymbol{y})$ continue dans $\Omega_{0}$, pour lesquels

$$
\text { (4.2.1) } \left.T=D_{x}^{\boldsymbol{p}} D_{\boldsymbol{y}}^{\boldsymbol{\alpha}} F \quad \text { et } \quad F(\boldsymbol{x}, \boldsymbol{y})=\frac{\left(\boldsymbol{x}-\boldsymbol{x}_{0}\right)^{\boldsymbol{p}}}{\boldsymbol{p} !} g(\boldsymbol{y})+o\left(\mid \boldsymbol{x}-\boldsymbol{x}_{0}\right)^{|\boldsymbol{p}|}\right)
$$

dans $\Delta \times \Omega_{0}$ (resp. dans $\left.\left(\Delta-\left\{\boldsymbol{x}_{0}\right\}\right) \times \Omega_{0}\right)$. Si $S(\boldsymbol{y})=T\left(\boldsymbol{x}_{0}, \boldsymbol{y}\right)$ (resp. $S(\boldsymbol{y})=$ $\left.=\lim _{x \rightarrow x_{0}} T(\boldsymbol{x}, \boldsymbol{y})\right)$ dans $\Omega_{0}$, on a alors

$\lim _{x \rightarrow x_{0}}$

$$
S=D^{\boldsymbol{\alpha}} g \quad \text { dans } \quad \Omega_{0} \text {. }
$$

Remarque 1. Dans le théorème 1 on peut exiger que la fonction $g$ soit donnée (dans $\Omega_{0}$ ), pourvu qu'elle soit continue et satisfasse à (4.2.2).

Dans le cas de la valeur (ou de la limite en un point) on a l'énoncé suivant (qui coincide avec celui de [5], 2 , dans le cas d'une variable):

TEÉORÈME 1'. Soit $T(x)$ une distribution définie dans un voisinage de $\boldsymbol{x}_{0}$ (resp. dans un voisinage de $\boldsymbol{x}_{0}$ pour $\boldsymbol{x} \neq \boldsymbol{x}_{0}$ ). Pour que la valeur $T\left(x_{0}\right)=C$ (resp. la limite $\lim T(x)=C$ ) existe, il faut et il suffit qu'il existe un $\boldsymbol{p} \epsilon \bar{C}_{0}^{m}$ et une fonction continue $F$ tels que

$$
T=D^{\boldsymbol{p}} F \quad \text { et } \quad F(\boldsymbol{x})=C \frac{\left(\boldsymbol{x}-\boldsymbol{x}_{0}\right)^{\boldsymbol{p}}}{\boldsymbol{p} !}+o\left(\left|\boldsymbol{x}-\boldsymbol{x}_{0}\right|^{|\boldsymbol{p}|}\right)
$$

dans un voisinage de $\boldsymbol{x}_{0}$ (resp. dans un voisinage de $\boldsymbol{x}_{0}$ pour $\boldsymbol{x} \neq \boldsymbol{x}_{0}$ ). 
On a naturellement des théorèmes analogues dans le cas de la limite suivant un cône.

Remarque 2. Dans les théorèmes 1 et $1^{\prime}$ on peut exiger que l'on ait $\boldsymbol{p} \geqslant \boldsymbol{p}_{0}, \boldsymbol{q} \geqslant \boldsymbol{q}_{0}\left(\boldsymbol{p}_{0}, \boldsymbol{q}_{0}\right.$ étant donnés $)$.

Finalement, le corollaire du $\mathrm{N}^{0}$ précédent conduit au théorème suivant:

THस́⿴囗十े̀me 2. Soit $\lambda_{v}$ une suite décroissante qui satisfait à (4.1.1). On peut remplacer dans les définitions $d u$ $\$ 3$ la condition.

par

$$
\lim _{\lambda \rightarrow 0+} T\left(\boldsymbol{x}_{0}+\lambda \boldsymbol{x}, \boldsymbol{y}\right)=S(\boldsymbol{y}) \quad\left(\text { resp. } \lim _{\lambda \rightarrow 0+} T\left(\boldsymbol{x}_{0}+\lambda \boldsymbol{x}\right)=C\right)
$$

$$
\lim _{\boldsymbol{y} \rightarrow \infty} T\left(\boldsymbol{x}_{0}+\lambda_{\nu} \boldsymbol{x}, \boldsymbol{y}\right)=S(\boldsymbol{y}) \quad\left(\text { resp. } \lim _{\boldsymbol{r} \rightarrow \infty} T\left(\boldsymbol{x}_{0}+\lambda_{\boldsymbol{v}} \boldsymbol{x}\right)=C\right) .
$$

4.3. Fixation dans la primitive et dans la dérivée. Le théorème 1 du $\mathrm{N}^{0}$ précédent permet de tirer des conclusions sur la possibilité de fixer des variables dans la primitive et dans la dérivée.

THÉ́noR̀̀m. Supposons que dans une distribution $T(\boldsymbol{x}, \boldsymbol{y})$ on puisse fixer $\boldsymbol{x}=\boldsymbol{x}_{0}$ sur un ouvert $\Omega$ de $\left(\varepsilon^{n}\right)_{\boldsymbol{y}}$ et soit $\Omega_{0}$ un ouvert borné tel que $\bar{\Omega}_{0} \subset \Omega$. Alors il en est de même sur $\Omega_{0}$

$1^{\circ}$ pour une primitive $T_{1}$ par rapport à $x_{i}$, où $T_{1}\left(\boldsymbol{x}_{0}, \boldsymbol{y}\right)$ peut être une distribution arbitraire (sur $\Omega_{0}$ );

$2^{\circ}$ pour toute primitive $T_{1}$ par rapport à $x$, dans le cas ò̀ $m=1$;

$3^{\circ}$ pour une primitive $T_{1}$ par rapport d̀ $y_{j}$, où $T_{1}\left(\boldsymbol{x}_{0}, \boldsymbol{y}\right)$ peut être une primitive arbitraire de $T\left(\boldsymbol{x}_{0}, \boldsymbol{y}\right)^{\prime}$ (sur $\Omega_{0}$ par rapport $\dot{a} y_{j}$ );

$4^{\circ}$ pour la dérivée $\frac{\partial}{\partial y_{j}} T$, ò̀ $\frac{\partial T}{\partial y_{j}}\left(\boldsymbol{x}_{0}, \boldsymbol{y}\right)=\frac{\partial}{\partial y_{j}}\left(T\left(\boldsymbol{x}_{0}, \boldsymbol{y}\right)\right)$.

Démonstration. Soient $\boldsymbol{p}, \boldsymbol{q}, T, S, F, g$ comme dans le théorème 1 du $\mathrm{N}^{0} 4.2$.

Posons $\overline{\boldsymbol{p}}=\left(p_{1}, \ldots, p_{i}-1, \ldots, p_{m}\right)$, alors $T_{0}=D_{\boldsymbol{x}}^{\overline{\boldsymbol{p}}} D_{\boldsymbol{y}}^{\boldsymbol{\alpha}} F$ est une primitive de $T$ par rapport à $x_{i}$; d'après (4.2.1), on a $F(\boldsymbol{x}, \boldsymbol{y})=o\left(\left|\boldsymbol{x}-\boldsymbol{x}_{0}\right|^{|\overline{\boldsymbol{p}}|}\right)$, d'où $T_{\mathbf{0}}\left(\boldsymbol{x}_{0}, \boldsymbol{y}\right)=0$ dans $\Omega_{0} ; S_{1}(\boldsymbol{y})$ étant une distribution arbitraire sur $\Omega_{0}$, la distribution $T_{1}(\boldsymbol{x}, \boldsymbol{y})=T_{0}(\boldsymbol{x}, \boldsymbol{y})+S_{1}(\boldsymbol{y})$ est aussi une primitive par rapport à $x_{i}$ et on a $T_{1}\left(\boldsymbol{x}_{0}, \boldsymbol{y}\right)=S_{1}(\boldsymbol{y})$. Si $m=1$ et si $T_{2}$ est une primitive quelconque de $T$ par rapport à $x$, on a $T_{2}-T_{0}=S_{2}(y)$ donc
$T_{2}\left(x_{0}, y\right)_{4}=S_{2}(y)$ dans $\Omega_{0}$.

Posons $\overline{\boldsymbol{q}}=\left(q_{1}, \ldots, q_{j}-1, \ldots, q_{n}\right)$, alors $T_{3}=D_{\boldsymbol{x}}^{\boldsymbol{p}} D_{\boldsymbol{x}}^{\bar{\alpha}} F$ est une primitive de $T$ par rapport à $y_{i} ;$ d'après $(4.2 .1)$ et $(4.2 .2)$, on à $T_{3}\left(\boldsymbol{x}_{0}, \boldsymbol{y}\right)$ $=\bar{S}(\boldsymbol{y})$ dans $\Omega_{0}$, où $\bar{S}(\boldsymbol{y})=\overline{D_{\boldsymbol{y}}^{\boldsymbol{q}}} g(\boldsymbol{y}) ;$ si $\partial S_{3} / \partial y_{j}=S$, alors $\partial\left(S_{3}-\bar{S}\right) / \partial y_{j}$ $=0$, donc la distribution $T_{4}(\boldsymbol{x}, \boldsymbol{y})=T_{3}(\boldsymbol{x}, \boldsymbol{y})+S_{3}(\boldsymbol{y})-\bar{S}(\boldsymbol{y})$ est aussi une primitive par rappert à $y_{j}$ et on a $T_{4}\left(\boldsymbol{x}_{0}, \boldsymbol{y}\right)=S_{3}(\boldsymbol{y})$.
D'après la définition de la fixation des variables, nous arons $T\left(x_{0}+\lambda \boldsymbol{x}, \boldsymbol{y}\right) \rightarrow S(y)$ pour $\lambda \rightarrow 0+$. En différentiant par rapport à $y_{j}$, on obtient

$$
\frac{\partial}{\partial y_{j}}\left(x_{0}+\lambda \boldsymbol{x}, \boldsymbol{y}\right) \rightarrow \frac{\partial S}{\partial y_{j}}(\boldsymbol{y}) \quad \text { pour } \quad \lambda \rightarrow 0+,
$$

d'où il résulte que

$$
\frac{\partial T}{\partial y_{j}}\left(\boldsymbol{x}_{0}, \boldsymbol{y}\right)=\frac{\partial S}{\partial y_{j}}(\boldsymbol{y})
$$

Le théorème est ainsi complètement démontré.

\section{On a un théorème analogue pour la limite.}

4.4. Relation entre l'existence de la limite et la possibilité de fixer des variables. Considérons une distribution $\Sigma(\boldsymbol{x}, \boldsymbol{y})$ de support contenu dans l'hyperplan $x=0$. D'après un théorème de $\mathrm{L}$. Schwartz ([7], tome I, p. 100 et p. 113) elle est localement de la forme $\Sigma(\boldsymbol{x}, \boldsymbol{y})$ $=\sum_{\boldsymbol{p}} D_{\boldsymbol{x}}^{\boldsymbol{p}} \delta(\boldsymbol{x}) \times U_{\boldsymbol{p}}(\boldsymbol{y})$ (la somme étant finie). On a alors

$$
\Sigma(\lambda \boldsymbol{x}, \boldsymbol{y})=\sum_{\boldsymbol{p}} \frac{1}{\lambda^{|\boldsymbol{p}|+m}} D_{\boldsymbol{x}}^{\boldsymbol{p}} \delta(\boldsymbol{x}) \times U_{\boldsymbol{p}}(\boldsymbol{y}) .
$$

Il en résulte que si $\lim \lambda^{m} \Sigma(\lambda x, y)=0$, on a $\Sigma=0$. Nous dirons qu'une distribution ne possède pas de masse sur l'hyperplan $\boldsymbol{x}=\boldsymbol{x}_{0}$, si

$$
\lim _{\lambda \rightarrow 0+} \lambda^{m} T_{0}\left(x_{0}+\lambda x, y\right)=0 .
$$

Nous avons done le

LEMME. Chaque distribution $T(x, y)$ ne peut être représenté que d'une seule manière sous la forme

$$
T=T_{0}+\Sigma
$$

ò̀ $T_{0}$ ne possède pas de masse sur $\boldsymbol{x}=\boldsymbol{x}_{0}$ et $\Sigma(\boldsymbol{x}, \boldsymbol{y})=0$ pour $\boldsymbol{x} \neq \boldsymbol{x}_{0}$.

Remarque. Une distribution $T_{0}$ ne possède pas de masse sur $\boldsymbol{x}=\boldsymbol{x}_{0}$, si l'on peut fixer $\boldsymbol{x}=\boldsymbol{x}_{0}$ dans $T_{0}$; plus généralement, si $T_{0}(\boldsymbol{x}, \boldsymbol{y})$ $=D_{\boldsymbol{x}}^{\boldsymbol{p}} T_{\mathbf{1}}(\boldsymbol{x}, \boldsymbol{y})$, où $|\boldsymbol{p}| \leqslant m$ et $T_{\mathbf{1}}$ est une distribution dans laquelle on peut fixer $\boldsymbol{x}=\boldsymbol{x}_{0}{ }^{11}$ ).

Soit $G$ un ouvert de $\left(\varepsilon^{m+n}\right)_{x, y}$. Désignons par $\Omega$ l'ensemble des $\boldsymbol{y}$ tels que $\left(x_{0}, \boldsymbol{y}\right) \epsilon G$.

11) On voit à l'exemple de la distribution $T=\frac{d}{d x}(\ln |\ln | x||)$ que cette condition n'est pas nécessaire dans le cas $m=1$. Cependant on montre qu'elle est nécessaire dans le cas où $m>1$. 
CoRoliaIRe 1. Supposons que $T_{1}, T_{2}$ soient deux distributions définies dans $G$, dans lesquelles on puisse fixer $\boldsymbol{x}=x_{0}$ sur $\Omega$. Si $T_{1}=T_{2}$ dans $G$ pour $x \neq x_{0}$, alors $T_{1}=T_{2}$ dans $G$.

Le théorème dü $\mathrm{N}^{0} 4.2$ conduit à la proposition suivante:

Propositron 1. Soit $T$ une distribution définie dans $G-\left(\left\{\boldsymbol{x}_{\mathbf{0}}\right\} \times \Omega\right)$. si la limite $\lim T(\boldsymbol{x}, \boldsymbol{y})$ existe dans $\Omega$ alors il existe une et une seule.

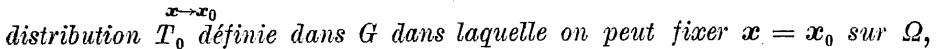
telle que $T=T_{\mathbf{0}}$ dans $G-\left(\left\{x_{0}\right\} \times \Omega\right)$. Alors $\lim T(\boldsymbol{x}, \boldsymbol{y})=T_{0}\left(\boldsymbol{x}_{0}, \boldsymbol{y}\right)$ dans $\Omega$ et on a la décomposition (4.4.2).

En effet, grâce au corollaire 1, on peut définir $T_{0}$ localement (cf. [6], tome I, p. 26) dans un ouvert contenant $\left\{x_{0}\right\} \times \Omega$ par $T_{0}=D_{x}^{\boldsymbol{p}} D_{\boldsymbol{y}}^{\boldsymbol{q}} F$, où $F$ est la même que dans le théorème 1 du No $\mathrm{N}^{0} .2$.

Dans le cas de la valeur $(n=0)$ nous avons l'énoncé (cf. [4], №3.3) suivant:

Proposimion 1'. Si la limite $\lim _{\boldsymbol{x} \rightarrow \boldsymbol{x}_{0}} T(\boldsymbol{x})$ existe, alors il existe une et une seule distribution $T_{0}$ possédant une valeur au point $x_{0}$ et telle que $T=T_{0}$ pour $x \neq x_{0}$.

Soit $T$ une distribution définie dans $G$. En combinant la proposition 1 et le lemme on obtient facilement les corollaires suivants:

CoRollatre 2. Si $T=D_{\boldsymbol{x}}^{\boldsymbol{p}} T_{1}$ dans $G-\left(\left\{\boldsymbol{x}_{0}\right\} \times \Omega\right)$, où. $|\boldsymbol{p}| \leqslant m$ et la limite $\lim T_{1}(\boldsymbol{x}, \boldsymbol{y})$ existe sur $\Omega$, alors il existe une et seule décomposition $\underset{\substack{x \rightarrow x_{0} \\(4.4 .2)}}{ }$

Corollatre 3. Si $T$ ne possède pas de masse sur $\boldsymbol{x}=\boldsymbol{x}_{0}$ et si la limite $\lim T(\boldsymbol{x}, \boldsymbol{y})$ existe sur $\Omega$, on peut fixer $\boldsymbol{x}=\boldsymbol{x}_{0}$ dans $T$ sur $\Omega$ et on a $T\left(\boldsymbol{x}_{0}, \boldsymbol{y}\right)=\lim _{\boldsymbol{x} \rightarrow x_{0}} T(\boldsymbol{x}, \boldsymbol{y})$

On obtient enfin par la même voie une proposition sur le recollement analogue à celle de [5], No 3.3 :

Proposition 2. Admettons $n=1$ et soient $T_{1}, T_{2}$ des distributions définies dans $G$ pour $x<x_{0}$ resp. pour $x>x_{0}$. Si $T_{i}=\partial S_{i} / \partial x(i=1,2)$, où $S_{i}$ sont des distributions pour lesquelles les limites $\lim _{x \rightarrow x_{0}-} S_{1}$ et $\lim _{x \rightarrow x_{0+}} S_{2}$ existent sur $\Omega$, alors il existe une et une seule distribution $T$ définie dans $G$, telle que $T=T_{1}$ pour $x<x_{0}, T=T_{2}$ pour $x>x_{0}$ et telle que $T=\partial S / \partial x$, où $S$ est une distribution dans laquelle on peut fixer $x=x_{0}$ sur $\Omega^{12}$ ).

12) On montre que cette dernière condition (sur $T$ ) équivaut à celle de l'existence du produit multiplicatif (ef. [4]) TH $\left(x-x_{0}\right)$, où $H$ désigne la fonction d'Heaviside.
4.5. Autre forme de la définition de la fixation. Traduisons maintenant les définitions du $\$ 3$ en nous servant de l'égalité

$$
\left.T\left(x_{0}+\lambda x, y\right), \chi(x, y)\right)=\left(T, \frac{1}{\lambda^{n}} \chi\left(\frac{x-x_{0}}{\lambda}, y\right)\right) .
$$

Nous obtenons ainsi les énoncés que voici.

Pour que la section $T\left(x_{0}, \boldsymbol{y}\right)=S(\boldsymbol{y})$ sur $\varrho \quad$ (resp. la limite $\left.\lim _{x \rightarrow x_{0}} T(\boldsymbol{x}, \boldsymbol{y})=S(\boldsymbol{y})\right)$ existe, il faut et il suffit qu'on ait

$$
\lim _{\lambda \rightarrow 0+}\left(T, \frac{1}{\lambda^{m}} \chi\left(\frac{x-x_{0}}{\lambda}, y\right)\right)=\left(S, \int \chi(x, y) d x\right)_{y}
$$

pour tout $\chi \in \mathcal{D}_{\left(c^{c m}\right) \times \Omega}$ (resp. $\left.\chi \in D_{\left(-\left\{x_{0}\right\}\right) \times \Omega}\right)$.

Pour que la valeur $T\left(x_{0}\right)=C$ (resp. la limite $\lim T(x)=C$ ) existe, il faut et il suffit qu'on ait

$$
\lim _{\lambda \rightarrow 0+}\left(T, \frac{1}{\lambda^{m}} \varphi\left(\frac{x-x_{0}}{\lambda}\right)\right)=C
$$

pour tout $\varphi \in \mathcal{D}$ (resp. $\varphi \in \cdot)_{\left(-\left\{x_{0}\right\}\right)}$ ) tel que $\int \varphi d x=1$.

Or il suffit que la condition (4.5.1) soit remplie pour les fonctions $\chi(\boldsymbol{x}, \boldsymbol{y})$ de la forme $\varphi(\boldsymbol{x}) \psi(\boldsymbol{y})$. Nous arons, en effet:

THÉORÈME. Pour que la section $T\left(\boldsymbol{x}_{0}, \boldsymbol{y}\right)=S(\boldsymbol{y})$ sur $\Omega$ existe (où $\Omega$ est un ouvert de $\left.\left(\mathcal{E}^{n}\right)_{y}\right)$ il faut et il suffit qu'on ait

$$
\lim \left(T, \frac{1}{\lambda^{m}} \varphi\left(\frac{x-x_{0}}{\lambda}\right) \psi(y)\right)=(S, \psi)_{y}
$$

pour $\psi \in D_{\Omega}$ et $\varphi \in D_{\boldsymbol{x}}$ tel que $\int \varphi d \boldsymbol{x}=1$.

Autrement dit, pour que l'on puisse fixer $x=x_{0}$ sur $\Omega$ dans la distribution $T$ il faut et il suffit que pour tout $\psi \boldsymbol{\epsilon} \mathcal{D}_{\Omega}$ la distribution $U_{\psi}(\boldsymbol{x})=$ $=\left(T(\boldsymbol{x}, \boldsymbol{y}),\left.\psi(\boldsymbol{y})\right|_{\boldsymbol{y}}\right.$ (cf. $\left.\mathbf{N}^{0} 1.3\right)$ possède une valeur au point $\boldsymbol{x}_{0}$. On a alors

$$
(4.5 .4) \quad\left(T\left(\boldsymbol{x}_{0}, \boldsymbol{y}\right), \psi(\boldsymbol{y})\right)_{\boldsymbol{y}}=U_{\psi}\left(\boldsymbol{x}_{0}\right) \text {. }
$$

Nous démontrerons que la condition (4.5.3) est suffisante (sa nécessité étant évidente) dans le $N^{0} 8.2$. En ce qui concerne le passage du premier énoncé au second, il suffit d'observer que si pour tout $\psi \epsilon \mathcal{D}_{\Omega}$ la distribution $\left(S^{\prime}(\boldsymbol{x}, \boldsymbol{y}), \psi(\boldsymbol{y})\right)_{\boldsymbol{y}}$ est constante, alors la distribution $S(\boldsymbol{x}, \boldsymbol{y})$ ne dépend pas de $\boldsymbol{x}$ pour $\boldsymbol{y} \in \Omega$.

On sait (cf. $\mathrm{N}^{0}$ 1.2) que la convergence dans (3.2) entraîne la convergence uniforme de $\left(T\left(\boldsymbol{x}_{0}+\lambda \boldsymbol{x}, \boldsymbol{y}\right), \varphi(\boldsymbol{x}, \boldsymbol{y})\right)$ dans l'ensemble $\left|D^{\boldsymbol{p}} \varphi\right| \leqslant 1$, $\varphi \in \mathscr{D}_{E}$, où $\boldsymbol{p}=\boldsymbol{p}(E)$ et est un compact arbitraire contenu dans le do- 
maine de convergence dans (3.2). Ainsi nous obtenons facilement les énoncés suivants.

Désignons par $P_{\lambda}\left(x_{0}\right)$ le, cube $\left|x_{i}-x_{i 0}\right|<\lambda(i=1, \ldots, m)$.

Proposmion 1. Si l'on peut fixer des variables: $T\left(\boldsymbol{x}_{0}, \boldsymbol{y}\right)=S(\boldsymbol{y})$ ur alors pour tout ouvert borné $\Omega_{0}$ tel que $\bar{\Omega}_{0} \subset \Omega$ il existe un $(\boldsymbol{p}, \boldsymbol{q}) \epsilon \Upsilon_{0}^{m+n}$ tel que pour chaque $\psi \epsilon^{\mathcal{D}_{\Omega}^{\alpha}}$ on a

$$
(4.5 .5) \quad(T, \chi(\boldsymbol{x}, \boldsymbol{y})) \rightarrow(S, \psi(\boldsymbol{y})) \text { pour } \quad \lambda \rightarrow 0+,
$$

lorsque

(4.5.6) $\chi \in \mathcal{D}_{\boldsymbol{P}_{\lambda}\left(x_{0}\right) \times \Omega_{0}}^{\boldsymbol{p}, \boldsymbol{q}}, \int D_{\boldsymbol{y}}^{\boldsymbol{q}} \chi(\boldsymbol{x}, \boldsymbol{y}) d \boldsymbol{x} \rightarrow D_{\boldsymbol{y}}^{\boldsymbol{q}} \psi(\boldsymbol{y})$ uniformément (par rapport

$$
\grave{a} \boldsymbol{y}) \text { et } \sup \left|D_{\boldsymbol{x}}^{\boldsymbol{p}} D_{\boldsymbol{y}}^{\boldsymbol{q}} \chi\right|=0\left(\frac{1}{\lambda^{m+|\boldsymbol{p}|}}\right) \text {. }
$$

En particulier on a pour tout $\psi \in \mathcal{D}_{\Omega_{0}}^{\boldsymbol{p}_{0}}$

$$
(T, \varphi(\boldsymbol{x}) \psi(\boldsymbol{y})) \rightarrow(S, \psi(\boldsymbol{y})) \quad \text { pour } \quad \lambda \rightarrow 0+
$$

lorsque

$$
\text { (4.5.8) } \quad \varphi \in \mathcal{D}_{\boldsymbol{P}_{\hat{\lambda}}\left(x_{0}\right)}^{\boldsymbol{p}_{1}}, \quad \int \varphi d \boldsymbol{x}=1 \quad \text { et } \quad \sup \left|D_{\boldsymbol{x}}^{\boldsymbol{p}} \varphi\right|=O\left(\frac{1}{\lambda^{m+|\boldsymbol{p}|}}\right) \text {. }
$$

Proposition 1'. Si $T\left(\boldsymbol{x}_{0}\right)=C$, alors il existe un $\boldsymbol{p} \epsilon \boldsymbol{T}_{0}^{m}$ tel que $(T, \varphi) \rightarrow C$. pour $\lambda \rightarrow 0+$ lorsque $\varphi$ satisfait $\grave{a}$ (4.5.8).

On a naturellement des théorèmes analogues dans le cas de la limite. Nous démontrerons des théorèmes plus précis dans le $\$ 8$.

4.6. Cas d'une mesure et d'une fonction. Soit $\mu$ une mesure définie dans un voisinage de 0 dans $\varepsilon^{m}$. On sait que

$$
\text { (4.6.1) } \quad \mu=D^{1} f, \quad \text { où } \quad f(x)=\operatorname{sign} x^{1} \mu\left(\left[0, x_{1}\right) \times \ldots \times\left[0, x_{m}\right)\right)
$$

(on pose $[0, x)=[x, 0$ ), si $x<0$ ). Il en résulte que si $\mu(\{0\})=0$, on a $\lim f(x)=0$ et (d'après la remarque du $\left.\mathrm{N}^{0} 4.4\right) \mu$ ne possède pas de masse

au point $x_{0}$. Selon le corollaire $2 \mathrm{du} \mathrm{N}^{0} 4.4$ nous avons donc la

Proposimion 1 . Si $\mu$ est une mesure (dans un voisinage de $\boldsymbol{x}_{0}$ ) alors il existe une et une seule décomposition (4.4.2) et elle prend la forme $\mu=\mu_{0}+c \delta\left(\boldsymbol{x}-\boldsymbol{x}_{0}\right)$, où $c=\mu\left(\left\{\boldsymbol{x}_{0}\right\}\right)$. Pour qu'une mesure $\mu$ ne possède pas de masse en $\boldsymbol{x}_{0}$ il faut et il suffit que $\mu\left(\left\{\boldsymbol{x}_{0}\right\}\right)=0$.

D'après ce qui précède, le corollaire $3 \mathrm{du} \mathrm{N}^{0} 4.4$ entraîne la

Proposinion 2. Dans le cas d'une fonction sommable dans un voisinage de $\boldsymbol{x}_{0}$ la notion de limite au point $\boldsymbol{x}_{0}$ coïncide avec celle de valeur au point $x_{0}$.

Supposons maintenant qu'une mesure $\mu$ possède la densité 0 au point $\mathbf{0}$. On vérifie que $f(\boldsymbol{x})=o\left(|\boldsymbol{x}|^{m}\right)$ pour la fonction $f(\boldsymbol{x})$ définie par la formule $\left.(4.6 .1)^{13}\right)$. Si l'on prend

on trouve

$$
F(\boldsymbol{x})=\int_{0}^{x_{1}} \ldots \int_{0}^{x_{m}} f(\boldsymbol{x}) d x_{1} \ldots d x_{m},
$$

$$
\frac{\partial^{2 m}}{\partial x_{1}^{2} \ldots \partial x_{m}^{2}} F=\mu
$$

et $F^{\prime}(\boldsymbol{x})=o\left(|\boldsymbol{x}|^{2 m}\right)$, d'où il résulte, selon le théorème $1^{\prime} \mathrm{du} \mathrm{N}^{0} 4.2$, que $\mu(0)=0$. Nous avons donc la

Proposition 3. Si une mesure possède une densité au point $x_{0}$, elle possède aussi une valeur au point $\boldsymbol{x}_{0}$ égale à la densité en ce point.

Corolualre 1. Une fonction localement sommable $f(x)$ possède une valeur (au sens des distributions) en chaque point $x_{0}$ pour lequel la limite

$$
\lim \frac{1}{|I|} \int_{I} f(\boldsymbol{x}) \boldsymbol{d} \boldsymbol{x}
$$

existe lorsque $I \rightarrow x_{0}$ régulièrement (cf. $\mathrm{N}^{0} 1.1$ ); la valeur est alors égale $\grave{a}$ cette limite.

COROLlaTRE 2. Une mesure possède une valeur presque partout. Il en est de même d'une fonction localement sommable et la valeur au sens des distributions est égale presque partout à celle au sens usuel.

THÉоRÈmE. Dans le cas d'une mesure $\geqslant 0$ la notion de valeur (en un point) coüncide avec celle de densité.

Démonstration. En vertu de la proposition 3 il reste à montrer que l'existence de la valeur entraîne celle de la densité. Supposons qu'une mesure $\mu \geqslant 0$ possède la valeur $\mu(0)=C$. Désignons par $Q_{\lambda_{1}}, \ldots, \lambda_{m}(x)$ l'intervalle $\left[x_{1}-\lambda_{1} / 2, x_{1}+\lambda_{2} / 2\right] \times \ldots \times\left[x_{m}-\lambda_{m} / 2, x_{m}+\lambda_{m} / 2\right]$. Soit $\varepsilon>0$ et choisissons deux fonctions $\varphi, \psi \epsilon(\mathcal{D})_{x}$ telles que $0 \leqslant \varphi \leqslant 1$, support de $\varphi \subset Q_{1, \ldots, 1}(0), \psi \geqslant 0, \psi=1$ dans $Q_{1, \ldots, 1}(0)$ et

(4.6.3) $1-\varepsilon<\int \varphi d x<1<\int \psi d x<1+\varepsilon$.

On a alors

$$
\begin{aligned}
\left(\mu, \frac{1}{\lambda_{1} \ldots \lambda_{m}} \varphi\left(\frac{x_{1}-a_{1}}{\lambda_{1}}\right.\right. & \left.\left., \ldots, \frac{x_{m}-a_{m}}{\lambda_{m}}\right)\right) \leqslant \frac{\mu\left(Q_{\lambda_{1} \ldots, \lambda_{m}}(\boldsymbol{a})\right)}{\lambda_{1} \ldots \lambda_{m}} \\
& \leqslant\left(\mu, \frac{1}{\lambda_{1} \ldots \lambda_{m}} \psi\left(\frac{x_{1}-a_{1}}{\lambda_{1}}, \ldots, \frac{x_{m}-a_{m}}{\lambda_{m}}\right)\right) .
\end{aligned}
$$

13) On représente l'intervalle $\left[0, x_{1}\right) \times \ldots \times\left[0, x_{m}\right)$ par des intervalles pour lesquels les rapports mutuels des longueurs des arêtes sont $\leqslant 2$, en utilisant la formule $\left[0, x_{i}\right)=\left(\left[-\xi \operatorname{sign} x_{i}, x_{i}\right) \cup\left[0, \xi \operatorname{sign} x_{i}\right)\right)-[-\xi, \xi)$, où $\xi=\max \left|x_{i}\right|$. 
Supposons maintenant que $\lambda_{1} \rightarrow 0+, \ldots, \lambda_{n} \rightarrow 0+$ de façon que $0 \in Q_{\lambda_{1}, \lambda_{m}}(\boldsymbol{a})$ et que $\lambda_{i} / \lambda_{j}$ restent bornés. Selon la proposition $1^{\prime} \mathrm{du} \mathrm{N}^{0} 4.5$ $\mathbf{0} \in Q_{\lambda_{1}, \ldots, \lambda_{m}}(\boldsymbol{a})$ et que $\lambda_{i} / \lambda_{j}$ restent
les membres extrêmes de (4.6.4) tendent vers $C \int \varphi d \boldsymbol{x}$ et $C \int \psi d \boldsymbol{x}$. D'après (4.6.3) ( $\varepsilon$ étant arbitraire) il en résulte que

$$
\frac{\mu\left(Q_{\lambda_{1}, \ldots, \lambda_{m}}(\boldsymbol{a})\right)}{\lambda_{1} \ldots \lambda_{m}} \rightarrow C
$$

c. q. f. d.

COROLLATRE 3. Pour qu'une fonction sommable et bornée inférieurement ou supérieurement possède une valeur (au sens des distributions) au point $x_{0}$ il faut et il suffit que la limite (4.6.2) existe.

En général on peut s'attendre à ce qu'on ait la formule (cf. [5], No 2.9)

$$
f(0)=\lim \frac{\left(p_{1}+1\right) \ldots\left(p_{m}+1\right)}{\boldsymbol{x}^{p+x}} \int_{0}^{x_{1}} \ldots \int_{0}^{x_{m}} f(\boldsymbol{u})(\boldsymbol{x}-\boldsymbol{u})^{\boldsymbol{p}} d \boldsymbol{u},
$$

lorsque $\boldsymbol{x} \rightarrow \mathbf{0}$ de façon que $\left|x_{i}\right| x_{j} \mid$ restent borné,

pour un $\boldsymbol{p}$. En effet, on vérifie (en s'appuyant sur lë théorème $1^{\prime}$ du $\mathrm{N}^{0} 4.2$ ) que l'existence de la limite $\left(4.6 .5\right.$ ) (où $\left|x_{i}\right| x_{j} \mid \leqslant 2$, cf. renvoi ${ }^{13}$ ), p. 27) que l'existence de la limite (4.6.5 celle de la valeur et alors la formule (4.6.5) a lieu. Par contre l'assertion réciproque est fausse. Pour en donner un exemple, posons

$$
f(x, y)=\frac{\partial^{3} F(x, y)}{\partial x^{2} \partial y}
$$

où

$$
F(x, y)=\left\{\begin{array}{ccc}
0 & \text { pour } & y=0, \\
x y \alpha\left(x / y^{3}\right) & \text { pour } & y \neq 0,
\end{array} \text { et } \alpha(z)=\left\{\begin{array}{ccc}
0 & \text { pour } & |z|>1, \\
\left(1-z^{2}\right)^{4} & \text { pour } & |z| \leqslant 1
\end{array}\right.\right.
$$

On a done pour $y \neq 0$ :

$$
\begin{gathered}
\frac{\partial F}{\partial x}=y \alpha+\frac{x}{y^{2}} \alpha^{\prime}, \quad \frac{\partial^{2} F}{\partial x^{2}}=\frac{2}{y^{2}} \alpha^{\prime}+\frac{x}{y^{5}} \alpha^{\prime \prime}, \\
\frac{\partial^{3} F}{\partial x^{2} \partial y}=-\frac{4}{y^{3}} \alpha^{\prime}-\frac{11 x}{y^{6}} \alpha^{\prime \prime}-\frac{3 x^{2}}{y^{9}} \alpha^{\prime \prime \prime} ;
\end{gathered}
$$

on vérifie sans peine (en utilisant un théorème de [7], tome I, p. 58) que les dérivations usuelles coïncident avec celles au sens des distributions et que $f(x, y)$ est une fonction localement sommable (dans $\varepsilon^{2}$ ). Comme on a $F(x, y)=O\left(r^{3}\right)$ (où $r=\sqrt{x^{2}+y^{2}}$ ), $f$ possède la valeur 0 au point $(0,0)$. D'autre part, du fait que $\lim _{y \rightarrow 0} \frac{\partial^{2} F}{\partial x^{2}}=0($ pour $x \neq 0), \frac{\partial F}{\partial x}(0, y)=y$ (pour $y \neq 0$ ) et $f(0, y)=0$, on tire

$$
\int_{0}^{y} \int_{0}^{x} f(\xi, \eta)(x-\xi) d \xi d \eta=F(x, y)-x y .
$$

$\mathrm{II}$ en résulte que pour $p \geqslant 1, q^{i} \geqslant 0$ on a

$$
\int_{0}^{x} \int_{0}^{y} f(\xi, \eta) \frac{(x-\xi)^{p}(y-\eta)^{q}}{(p-1) !(q-1) !} d \xi d \eta=\bar{F}(x, y)-\frac{x^{p} y^{q+1}}{p !(q+1) !}
$$

où $\bar{F}(x, y)=o\left(r^{p+q+2}\right)$, ce qui montre que la limite (4.6.5) n'existe pour aucun couple $(p, q) \epsilon \Upsilon_{0}^{2}$.

Passons maintenant au cas de la fixation des variables. Soit $G$ un ouvert de $\left(\mathcal{E}^{m+n}\right)_{\boldsymbol{x}, \boldsymbol{y}}$ et désignons par $\Omega_{\boldsymbol{x}}$ l'ensemble des $\boldsymbol{y}$ tels que $(\boldsymbol{x}, \boldsymbol{y})_{\epsilon} G$.

Les propositions 1 et 2 admettent des analogues. En effet, il suffit de montrer qu'une mesure $\mu(\boldsymbol{x}, \boldsymbol{y})$ définie dans $G$ ne possède pas de masse sur $\boldsymbol{x}=\boldsymbol{x}_{0}$ pourvu que $\mu(E)=0$ pour $E \subset\left\{\boldsymbol{x}_{0}\right\} \times \Omega_{x_{0}}$. Or, on a alors $|\mu|(E)=0$ pour $E \subset\left\{x_{0}\right\} \times \Omega_{x_{0}}$ et, par suite,

$$
\mid\left(\lambda^{m} \mu(\lambda \boldsymbol{x}, \boldsymbol{y}), \varphi(\boldsymbol{x}, \boldsymbol{y})|\leqslant|\left(|\mu|, \varphi\left(\frac{\boldsymbol{x}-\boldsymbol{x}_{0}}{\lambda}, \boldsymbol{y}\right)\right) \mid \rightarrow 0\right.
$$

lorsque $\lambda \rightarrow 0+\left(\right.$ pour $\left.\varphi \epsilon()_{\left(\mathcal{C}^{m}\right)_{x}} \times \Omega_{x_{0}}\right)$, d'où il résulte que

$$
\lim _{\lambda \rightarrow 0+} \lambda^{m} \mu(\lambda \boldsymbol{x}, \boldsymbol{y})=0 \text {. }
$$

On voit done que la condition que la mesure $\mu$ ne possède pas de masse sur $\boldsymbol{x}=\boldsymbol{x}_{0}$ équivaut à celle que $|\mu|\left(\left\{\boldsymbol{x}_{0}\right\} \times \Omega_{x_{0}}\right)=0$; aucune fonction ne possède de masse sur un hyperplan $\left.{ }^{14}\right)$.

Remarquons ensuite que la section d'une mesure $\geqslant 0$ est aussi une mesure $\geqslant 0$.

En vertu du théorème $d u \mathrm{~N}^{0} 4.5$, la proposition 3 et le théorème de ce $\mathrm{N}^{0}$ donnent

Proposition 4. Soit $\mu(\boldsymbol{x}, \boldsymbol{y})$ une mesure dans $G$. Pour que dans $\mu$ on puisse fixer $x=x_{0}$ sur $\Omega_{x_{0}}$ il suffit que pour tout $\psi \in \mathcal{D}_{\Omega_{x_{0}}}$ la mesure $\sigma_{\psi}=$

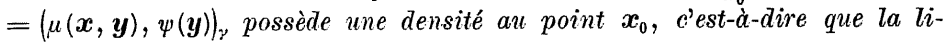

14) On vérifie qu'une distribution $T(x, y)$ qui est une fonction de $x$ (ef. $\mathrm{N}^{0} 1.4$ ) ne possède de masse sur aucun hyperplan $x=x_{0}$; en effet, d'après (1.4.1), on a alors

$$
\left|\lambda^{m}\left(T\left(\boldsymbol{x}_{0}+\lambda \boldsymbol{x}, \boldsymbol{y}\right), \varphi\right)\right| \leqslant\left|\lambda^{m} \int\left(S_{\boldsymbol{x}_{0}+\lambda \boldsymbol{x}}, \varphi(\boldsymbol{x}, \boldsymbol{y})\right)_{\boldsymbol{y}} d \boldsymbol{x}\right| \leqslant M \lambda^{m} \int_{P} g\left(\boldsymbol{x}_{0}+\lambda x\right) d \boldsymbol{x} \rightarrow 0
$$

où $P$ est un intervalle tel que $\left.\varphi \sigma^{c} D_{P \times\left(\mathcal{E}^{n}\right)}\right)_{y}$. 
mite (4.6.6) existe. Dans le cas où $\mu \geqslant 0$ cette condition est aussi nécessaire. On a, de plus,

(4.6.6) $\quad\left(\mu\left(\boldsymbol{x}_{0}, \boldsymbol{y}\right), \psi(\boldsymbol{y})\right)=\lim \frac{1}{|I|} \int_{I \times\left(\mathcal{C}^{m}\right) \boldsymbol{y}} \psi(\boldsymbol{y}) d \mu(\boldsymbol{x}, \boldsymbol{y})$

lorsque $I \rightarrow x_{0}$ régulièrement.

Propositron 5. Soit $\mu(\boldsymbol{x}, \boldsymbol{y})$ une mesure dans $G$. On peut fixer $\boldsymbol{x}=\boldsymbol{x}_{\mathbf{0}}$ sur $\Omega_{x_{0}}$ et $\mu\left(\boldsymbol{x}_{0}, \boldsymbol{y}\right)$. est une mesure dans $\Omega_{x_{0}}$ pour tout $\boldsymbol{x}_{0}$ tel $q u e \Omega_{\boldsymbol{x}_{0}} \neq 0$ sauf sur un ensemble des $x_{0}$ de mesure nulle.

Démonstration. On peut supposer, sans restreindre la généralité, que $G=P \times Q$, où $P \subset\left(\varepsilon^{m}\right)_{x}, Q \subset\left(\mathcal{E}^{n}\right)_{y}$ sont des intervalles et que $|\mu|(P \times Q)<\infty$. Si $I$ est un intervalle contenu dans $P$, la relation

$$
\left(\mu_{I}, \psi(\boldsymbol{y})\right)=\frac{1}{|I|} \int_{I \times Q} \psi(\boldsymbol{y}) d \mu(\boldsymbol{x}, \boldsymbol{y})
$$

définit évidemment une mesure $\mu_{I}$ dans $Q$ telle que $|\mu|_{I}(Q)<\infty$. En vertu de la proposition 4 il suffit de prouver que $\mu_{I}$ converge faiblement (cf. $\mathrm{N}^{0}$ 1.3) vers une mesure dans $Q$ lorsque $I \rightarrow x_{0}$ régulièrement, pour presque tous les $x_{0} \in P$. Les formules

$$
\sigma_{\psi}(E)=\int_{E \times Q} \psi(\boldsymbol{y}) d \mu(\boldsymbol{x}, \boldsymbol{y})
$$

et

$$
\sigma(E)=|\mu|(E \times Q)
$$

donnent des mesures sur $P$ telles que $\left|\sigma_{\psi}\right|(P)<\infty$ et $\sigma(P)<\infty$. En rapprochant (4.6.7), (4.6.8) et (4.6.9) on obtient

$$
\left(\mu_{I}, \psi\right)=\frac{1}{|I|} \sigma_{\psi}(I)
$$

et

(4.6.11)

$$
\left|\mu_{I}\right|(Q) \leqslant \frac{1}{|I|} \sigma(I)
$$

Soit $\left\{\psi_{p}\right\}$ une suite dense dans $\mathcal{D}_{Q}^{0}$. Sauf sur un ensemble des $\boldsymbol{x}_{0} \in Q$ de mesure nulle les limites $\lim \sigma_{\psi_{v}}(I) /(I)(v=1,2, \ldots)$ et $\lim \sigma(I) /|I|$ existent lorsque $I \rightarrow x_{0}$ régulièrement. Prenons un tel $x_{0}$ et supposons que $I \rightarrow x_{0}$ régulièrement; or, en vertu de (4.6.10), $\left(\mu_{I}, \psi_{v}\right)$ converge pour $\nu=1,2, \ldots$ et, d'après (4.6.11), $\left|\mu_{I}\right|(Q)$ reste borné, d'où il résulte que $\mu_{I}$ converge faiblement vers une mesure dans $Q$, c. q. f. d.
4.7. Cas d'une distribution qui est une fonction de $x$. Nous allons envisager les distributions de la forme

$$
T(\boldsymbol{x}, \boldsymbol{y})=S_{\boldsymbol{x}}(\boldsymbol{y})
$$

dans un ouvert $G$, où $S_{x}$ est une fonction distributionnelle localement sommable (cf. $\mathrm{N}^{0}$ 1.4). Cette classe de distributions contient les fonctions localement sommables. Désignons par $\Omega_{x}$ l'ensemble des $\boldsymbol{y}$ tels que $(\boldsymbol{x}, \boldsymbol{y}) \epsilon G$.

Revenons au théorème du $N^{0} 4.5$. D'après (4.7.1) on a

$$
\left(U_{p}, \varphi(\boldsymbol{x})\right)=(\boldsymbol{T}(\boldsymbol{x}, \boldsymbol{y}), \varphi(\boldsymbol{x}) \varphi(\boldsymbol{y}))_{\boldsymbol{x}, \boldsymbol{y}}=\int\left(\mathcal{S}_{\boldsymbol{x}}, \psi(\boldsymbol{y})\right)_{\boldsymbol{y}} \varphi(\boldsymbol{x}) d \boldsymbol{x},
$$

d'où il résulte que

$$
U_{\varphi}(\boldsymbol{x})=\left(S_{x}, \psi(\boldsymbol{y})\right)_{y}
$$

est une fonction localement sommable (dans l'ouvert des $\boldsymbol{x}$ donné par la condition $\{\boldsymbol{x}\} \times($ support de $\psi) \subset G$ ). Par conséquent la proposition 2 $\mathrm{du} \mathrm{N}^{0} 4.6$ donne la

Proposimion 1. Dans le cas d'une distribution de la forme (4.7.1), la notion de limite pour $\boldsymbol{x} \rightarrow x_{0}$ sur $\Omega_{x_{0}} \neq 0$ coïncide avec celle de section pour $\boldsymbol{x}=\boldsymbol{x}_{0}$ sur $\Omega_{\boldsymbol{x}_{0}}$. Il en est de même dans le cas d'une fonction sommable.

Pareillement, en vertu de (4.7.2), le corollaire $1 \mathrm{du}$ No 4.6 entraîne Propostrion 2. Si la limite

$$
\lim \frac{1}{|I|} \int_{I} S_{x} d x
$$

existe dans $\Omega_{x_{0}}$ lorsque $I \rightarrow x_{0}$ régulièrement, alors on peut fixer $\boldsymbol{x}=\boldsymbol{x}_{0}$ sur $\Omega_{x_{0}}$ et la section $T\left(\boldsymbol{x}_{0}, \boldsymbol{y}\right)$ est égale à cette limite dans $\Omega_{x_{0}}$.

Le théorème $d u \mathrm{~N}^{0} 4.5$ donne dans le cas de la limite (en vertu de (4.7.2)):

Propositron 3. Si la limite $\lim S_{x}$ existe dans $\Omega_{x_{0}}$ alors la limite

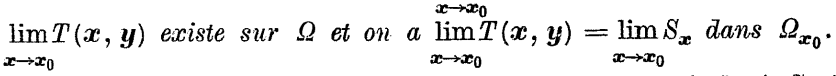

CoRollame 1. La section. $\boldsymbol{T}\left(\boldsymbol{x}_{0}, \boldsymbol{y}\right)$ existe et est égale à $\boldsymbol{S}_{\boldsymbol{x}_{0}}(\boldsymbol{y})$ pour tout point $x_{0}$ où la fonction distributionelle $S_{\boldsymbol{x}}$ est continue.

Considérons maintenant le cas $m=1$. Nous avons démontré dans [4], N02.9, que dans le cas d'une fonction sommable d'une variable l'existence de la limite (4.6.5) pour un $p$ est une condition nécessaire et suffisante pour l'existence de la valeur au point 0 ; la limite (4.6.5) existe, si la valeur est d'ordre $\leqslant p$. Il résulte de la proposition 1 du $\mathrm{N}^{0} 4.5$ que si la section $T\left(x_{0}, \boldsymbol{y}\right)$ existe sur un ouvert $\Omega$, alors pour tout ouvert borné $\Omega_{0}$ tel que $\bar{\Omega}_{0} \subset \Omega$ il existe un $p$ tel que pour $\psi \epsilon \mathcal{D}_{\Omega_{0}}$ la valeur 
de $U_{v}$ au point $x_{0}$ est d'ordre $\leqslant p$. En vertu de (4.7.2) nous avons done la condition nécessaire et suffisante que voici:

Proposition 4. Supposons $m=1$. Pour que la section $T\left(x_{0}, \boldsymbol{y}\right)$ $=S(y)$ existe sur un ouvert $\Omega \subset\left(\mathcal{E}^{n}\right)_{y}$, il faut et il suffit que pour tout ouvert borné $\Omega_{0}$ tel que $\bar{\Omega}_{0} \subset \Omega$ il existe un $p \in \bigcap_{0}$ tel que.

$$
S(\boldsymbol{y})=\lim _{x \rightarrow x_{0}} \frac{p+1}{\left(x-x_{0}\right)^{p}} \int_{x_{0}}^{x} S_{u}(x-u)^{p} d u \quad \text { dans } \quad \Omega_{0} .
$$

Supposons maintenant que $S_{x}=\mu_{x}$ soit une mesure $\geqslant 0$. alors $T=\mu=\mu_{x}(y)$ est une mesure $\geqslant 0$ et on a (et. $\mathrm{N}^{0} 1.4$ )

$$
\int_{I \times\left(\mathcal{C}^{\left.n n^{\prime}\right)} \boldsymbol{y}\right.} \psi(\boldsymbol{y}) d \mu(\boldsymbol{x}, \boldsymbol{y})=\int_{I}\left(\int \psi(\boldsymbol{y}) d \mu_{\boldsymbol{x}}\right) d x=\left(\int_{I} \mu_{\boldsymbol{x}} d x, \psi(\boldsymbol{y})\right) .
$$

La proposition $4 \mathrm{du} \mathrm{N}^{0} 4.6$ nous fournit done l'énoncé suivant:

Proposition 5. Pour qu'on puisse fixer $\boldsymbol{x}=\boldsymbol{x}_{0}$ sur $\Omega_{\boldsymbol{x}_{0}}$ dans $\mu$ il faut et il suffit que la limite faible (4.7.4) (cf. $\mathrm{N}^{0} 1.3$ ) existe dans $\Omega_{x_{0}}$. On a alors

$$
\mu\left(\boldsymbol{x}_{0}, \boldsymbol{y}\right)=\lim \frac{1}{|I|} \int_{I} \mu_{\boldsymbol{x}} d \boldsymbol{x}
$$

dans $\Omega_{x_{0}}$ lorsque $I \rightarrow x_{0}$ régulièrement.

CoRollatRe 2. Pour qu'on puisse fixer $\boldsymbol{x}=\boldsymbol{x}_{0}$ sur un ouvert $\Omega$ dans une fonction $f(\boldsymbol{x}, \boldsymbol{y})$ sommable et bornée inférieurement ou supérieurement il faut et il suffit que la limite (faible au sens des mesures)

$$
\lim \frac{1}{|I|} \int_{I} f(\boldsymbol{x}, \boldsymbol{y}) d \boldsymbol{x}
$$

existe dans $\Omega$ lorsque $I \rightarrow x_{0}$ régulièrement; la section (qui est toujours une mesure) est égale à cette limite.

Proposition 6. Si $T$ est une distribution de la forme (4.7.1) dans $G$, alors on peut fixer $\boldsymbol{x}=\boldsymbol{x}_{0}$ et on $a$

$$
T\left(\boldsymbol{x}_{0}, \boldsymbol{y}\right)=S_{\boldsymbol{x}_{0}}(\boldsymbol{y}) \quad \text { dans } \quad \Omega_{\boldsymbol{x}_{0}}
$$

pour presque tous les $x_{0}$ tels que $\Omega_{x_{0}} \neq 0$.

Démonstration. Soient $P \subset\left(\mathcal{E}^{m}\right)_{x}, Q \subset\left(\varepsilon^{n}\right)_{y}$ des intervalles ouverts tels que $\bar{P} \times \bar{\Omega} \subset G$. Il suffit de prouver (4.7.5) dans $Q$ pour presque tous les $x_{0} \epsilon P$. Il existe (cf. $\left.\mathrm{N}^{0} 1.4\right)$ un $k \epsilon \mathcal{X}_{0}$ et une fonction $g(\boldsymbol{y})$ sommable dans $P$ tels que

(4.7.6) $\left|\left(S_{x}, \psi(y)\right)_{y}\right| \leqslant g(x)$ dans $P$, lorsque $\psi \epsilon \mathcal{D}_{Q}^{k}$ et $\|\psi\|_{k} \leqslant 1$.
Il existe un ensemble $Z_{0}$ de mesure nulle tel que pour $x_{0} \in P-Z_{0}$ la quantité

$$
\frac{1}{|I|} \int_{I} g(x) d x
$$

reste bornée lorsque $I \rightarrow x_{0}$ régulièrement. Soit $\left\{\psi_{v}\right\}$ une suite dense dans l'espace de Banach $\mathcal{D}_{\bar{Q}}^{k} .\left(S_{\boldsymbol{x}}, \psi_{\boldsymbol{v}}(\boldsymbol{y})\right)_{\boldsymbol{y}}$ étant sommable (par rapport à $\boldsymbol{x}$ ) dans $P$, il existe un ensemble $Z_{v}$ de mesure nulle tel que pour $\boldsymbol{x}_{0} \in P-Z_{v}$ on a

$$
\left(S_{x_{0}}, \psi_{\nu}(\boldsymbol{y})\right)_{y}=\lim \frac{1}{|I|} \int_{I}\left(S_{u}, \psi_{\nu}(\boldsymbol{y})\right)_{y} d \boldsymbol{u}
$$

lorsque $I \rightarrow x_{0}$ régulièrement $(v=1,2, \ldots)$. Soit $x_{0} \in P-\bigcup_{i \mid 0}^{\infty} Z_{i}$ et supposons que $I \rightarrow x_{0}$ régulièrement. En vertu de (4.7.6) la norme

$$
\sup _{\|y\|_{k} \leqslant 1} \frac{1}{|I|} \int_{I}\left(S_{\boldsymbol{x}}, \psi(\boldsymbol{y})\right)_{\boldsymbol{y}} d \boldsymbol{x}
$$

reste bornée, done (4.7.7) entraîne la convergence

$$
\left(S_{\boldsymbol{x}_{0}}, \psi(\boldsymbol{y})\right)_{\boldsymbol{y}}=\lim \frac{1}{|I|} \int_{I}\left(S_{\boldsymbol{u}}, \psi(\boldsymbol{y})\right)_{\boldsymbol{y}} d \boldsymbol{u}
$$

lorsque $I \rightarrow x_{0}$ régulièrement pour tout $\psi \in \mathscr{D}_{\bar{Q}}^{k}$. Il en résulte, d'après la proposition 2, que $T\left(\boldsymbol{x}_{0}, \boldsymbol{y}\right)=S_{\boldsymbol{x}_{0}}(\boldsymbol{y})$ dans $Q$, c. q. f. d.

COROLlatre 3. Dans une fonction localement sommable $f(\boldsymbol{x}, \boldsymbol{y})$, pour presque tous les $x_{0}$, on peut fixer $x=x_{0}$ et la section coüncide avec la fonction $f\left(\boldsymbol{x}_{0}, \boldsymbol{y}\right)$.

ExEMPLEs. 1. Montrons que chaque distribution peut être (localement) section d'une fonction sommable. En effet, étant donnée une distribution $T(\boldsymbol{y})$, soit $Q$ un intervalle ouvert dont l'adhérence est contenue dans le domaine d'existence de $T$ et soit $\varphi \in(D)_{y}, \int \varphi(\boldsymbol{y}) d \boldsymbol{y}=1$. La fonction

$$
\tau(\varepsilon, \boldsymbol{y})=T * \frac{1}{\varepsilon^{m}} \varphi\left(\frac{\boldsymbol{y}}{\varepsilon}\right)
$$

est continue pour $0<\varepsilon<\varepsilon_{0}, \boldsymbol{y} \epsilon \bar{Q}$ et on a $\tau(\varepsilon, \boldsymbol{y}) \rightarrow T(\boldsymbol{y})$ dans $Q$ pour $\varepsilon \rightarrow 0$. Comme la fonction $\alpha(\varepsilon)=\int_{Q}|\tau(\varepsilon, \boldsymbol{y})| d \boldsymbol{y}$ est continue pour $0<\varepsilon<\varepsilon_{0}$, on peut choisir une fonction $\varepsilon(x)$ continue et croissante pour $x>0$, telle que $\alpha(\varepsilon(x))<1 / \sqrt{x}$ pour $0<x<x_{0}$. Il en résulte que la fonction $f(x, y)$ $=\tau(\varepsilon(x), \boldsymbol{y})$ est sommable dans $\left(-x_{0}, x_{0}\right) \times Q$ et on a $f(x, y) \rightarrow T$ dans $Q$ Studia Mathematica T. XrII 
lorsque $x \rightarrow 0$ ( $x$ étant considéré comme un paramètre), ce qui montre, d'après les propositions 1 et 3 , que $T$ est la section de $f(x, y)$ pour $x=0$ sur $Q$.

2. Considérons les noyaux

$$
\frac{1}{2 \pi} \frac{1-r^{2}}{1+r^{2}-2 r \cos \vartheta} \text { et } \frac{1}{2 \sqrt{\pi t}} e^{-x^{2} / 2 t} \text {. }
$$

des formules de Poisson pour la solution du problème de Dirichlet, resp. du problème de Cauchy pour l'équation de la chaleur. On vérifio que le premier de ces noyaux possède une limite pour $r \rightarrow 1-$ égale à $\delta(\gamma)$ et

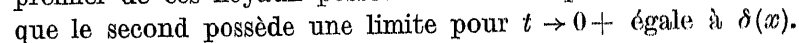

Pareillement le noyau

$$
u(t, x)=\left\{\begin{array}{lll}
\frac{1}{2} & \text { pour } & t>|x| \\
0 & \text { pour } & 0<t \leqslant|x|
\end{array}\right.
$$

de la formule de Kirchhoff pour la solution d'un problème do Canchy pour l'équation

$$
\frac{\partial^{2} u}{\partial t^{2}}=\frac{\partial^{2} u}{\partial x^{2}}
$$

possède la limite 0 pour $t \rightarrow 0+$, tandis que sa dérivée

$$
\frac{\partial u}{\partial t}=\frac{\delta(x+t)+\delta(x-t)}{2},
$$

qui est une distribution de la forme (4.7.1), possède une limite pour $t \rightarrow 0+$ égale à $\delta(x)$.

\section{§ 5. Détermination d'une distribution par ses valeurs}

5.1. Un lemme. Soit $\varphi(x)$ une fonction de $\varphi$ telle que $\psi \geqslant 0$ et $\int \varphi d x$ $=1$. Posons

$$
\varphi_{\lambda}(\boldsymbol{x})=\frac{1}{\lambda^{m}} \varphi\left(\frac{\boldsymbol{x}}{\lambda}\right) \quad \text { pour } \quad \lambda>0 .
$$

La démonstration du lemme qui suiti est la même que collo de [4], $\mathrm{N}^{0} 2.8$, dans le cas d'une variable.

LEMmE. Soit $S(\boldsymbol{x})$ une distribution et a $(\boldsymbol{x})$ une fonction non nérative de $D$ dont le support fait partie du domaine d'existence de $S$. $S i(S, a)<0$ alors pour tout $\lambda$ suffisamment petit il existe un point $\xi_{\lambda} \epsilon$ support de a tel $q u e\left(S, \varphi_{\lambda}\left(x-\xi_{\lambda}\right)\right)<0$.
5.2. Cas de la valeur. Les théorèmes qui suivent sont ceux de [5], $\S 5$, étendus au cas de plusieurs variables; les démonstrations restent les mêmes.

Supposons que les distributions $T$ et $S$ possèdent une valeur partout dans un ouvert $\left.G \subset\left(\mathcal{E}^{m}\right)_{\boldsymbol{x}}{ }^{15}\right)$. Soit $f(\boldsymbol{x})$ une fonction localement sommable dans $G$.

THÉoR:̀mE. Si $T(\xi) \geqslant f(\xi)$ presque partout dans $G$, alors $T \geqslant f$ dans $G$ (au sens des distributions) ${ }^{16}$ ).

CoROLLATRE 1. Si $T(\xi)=f(\xi)$ presque partout dans $G$, alors $T=f$ dans $G$. Si la valeur $T(\xi)$ existe partout dans $G$ et si elle est localement sommable comme fonction de $\xi$, alors $T$ coïncide avec cette fonction dans $G$.

CoROLLATRE 2. Si $T(\xi) \geqslant 0$ presque partout dans $G$, alors $T \geqslant 0$ dans $G$; si $T(\xi) \leqslant S(\xi)$ presque partout dans $G$, alors $T \leqslant S$ dans $G$.

COROLLATRE 3. Si $T(\xi)=0$ presque partout dans $G$, alors $T=0$ dans $G$; si $T(\xi)=S(\xi)$ presque partout dans $G$, alors $T=S$ dans $G$. La distribution qui possède une valeur partout est complètement déterminée par ses valeurs.

Remarque. Il suffit de supposer dans ces théorèmes que la valeur existe partout sauf sur un ensemble au plus dénombrable et que la condition (4.4.1) est satisfaite partout (c'est-à-dire qu'il n'y a de masse en aucun point). L'exemple de la distribution $\delta$ de Dirac montre qu'on ne peut pas supprimer la seconde de ces conditions. Pareillement on ne peut remplacer la première de ces conditions, même pas par l'hypothèse que l'ensemble exceptionnel soit de mesure linéaire nulle. En effet, soit $f(t)$ une fonction continue et croissante (strictement) dont la dérivée s'annule presque partout. La distribution

$$
T=\frac{\partial^{m}}{\partial x_{1} \ldots \partial x_{m}}\left[\min \left(f\left(x_{1}\right), \ldots, f\left(x_{m}\right)\right)\right]
$$

satisfait partout à la condition (4.4.1) (en vertu de la remarque du $\mathrm{N}^{0}$ 4.4). Elle est une mesure (de support situé sur la droite $x_{1}=\ldots=x_{m}$ ) dont la densité s'annule partout sauf sur un ensemble de la droite $x_{1}=\ldots=x_{m}$ de mesure linéaire nulle, par conséquent (cf. la propriété 3 du $\mathrm{N}^{0} 4.6$ ) elle possède une valeur égale à zéro partout sauf sur cet ensemble.

5.3. Cas de la fixation. Grâce au théorème du $N^{0} 4.5$ les théorèmes précédents s'étendent au cas de la fixation.

15) La valeur $T(\xi)$ comme fonction de $\xi$ est alors de I classe de Baire et elle possède la propriété suivante: pour tout $a$ chacun des ensembles $(\xi: T(\xi)>a)$ et $(\xi: T(\xi)<a)$ a un contingent complet en chacun de ses points.

16) La réciproque est évidente en vertu du corollaire $2 \mathrm{du} \mathrm{N}^{0} 4.6$. 
Soit $G$ un ouvert de $\left(\varepsilon^{n+n}\right)_{x, y}$ et désignons par $\Omega_{x}$ l'ensemble des $\boldsymbol{y}$ tels que $(\boldsymbol{x}, \boldsymbol{y}) \in G$. Supposons que dans les distributions $T(\boldsymbol{x}, \boldsymbol{y})$ et $\mathcal{S}(\boldsymbol{x}, \boldsymbol{y})$ on puisse fixer $\boldsymbol{x}=\boldsymbol{x}_{0}$ sur $\Omega_{\boldsymbol{x}_{0}}$ pour tout $\boldsymbol{x}_{0}$ (tel que $\Omega_{\boldsymbol{x}_{0}} \neq 0$ ) et désignons les sections par $T_{\boldsymbol{x}_{0}}(\boldsymbol{y})$ resp. $S_{\boldsymbol{x}_{0}}(\boldsymbol{y})$. Soit enfin $\Sigma$ une distribution de la forme $\Sigma(\boldsymbol{x}, \boldsymbol{y})=\Sigma_{\boldsymbol{x}}(\boldsymbol{y})$, où $\Sigma_{\boldsymbol{x}}(\boldsymbol{y})$ est une fonctionnelle localement sommable dans $G$. Nous avons le

THÉ́RÈmE. Si $T_{\boldsymbol{x}} \geqslant \Sigma_{\boldsymbol{x}}$ dans $\Omega_{\boldsymbol{x}}$ pour presque tous les $\boldsymbol{x}$ (tels que $\Omega_{\boldsymbol{x}} \neq 0$ ) alors $T \geqslant \Sigma$ dans $G$ et vice versa.

Démonstration. Soient $\left.\varphi \epsilon(D)_{x}, \psi \epsilon(D)\right)_{y}$ des fonctions non négatives telles que le support de $\varphi(\boldsymbol{x}) \psi(\boldsymbol{y})$ fasse partie de $G$. D'après le théorème du No4.5 la distribution $U_{\psi}$ possède une valeur en tout point $x$ d'un voisinage $d u$ support de $\varphi$ et on a

La fonction

$$
U_{\varphi}(x)=\left(T_{\boldsymbol{x}}, \psi\right)_{\boldsymbol{y}} .
$$

$$
f_{\psi}(\boldsymbol{x})=\left(\Sigma_{\boldsymbol{x}}, \psi\right)_{\boldsymbol{y}}
$$

est localement sommable dans un voisinage du support de $\varphi$. D'après les hypothèses, les égalités (5.3.1) et (5.3.2) donnent $U_{y}(\boldsymbol{x}) \geqslant f_{y}(\boldsymbol{x})$ presque partout, d'où il résulte, selon le théorème du $\mathrm{N}^{0} 5.2$, que $U_{v} \geqslant f_{\psi}$ dans un voisinage $\mathrm{du}$ support de $\varphi$. On a donc

$$
(T, \varphi(\boldsymbol{x}) \psi(\boldsymbol{y}))_{\boldsymbol{x}, \boldsymbol{y}}=\left(U_{\psi}, \varphi\right)_{\boldsymbol{x}} \geqslant\left(f_{\psi}, \varphi\right)_{\boldsymbol{x}}=(\Sigma, \varphi(\boldsymbol{x}) \psi(\boldsymbol{y}))_{\boldsymbol{x}, \boldsymbol{y}} .
$$

Il en résulte que $T \leqslant \Sigma$ dans $G$.

Le théorème réciproque résulte de la proposition $6 \mathrm{du} \mathrm{N}^{0} 4.7$.

COROLLATRE 1. Si $T_{\boldsymbol{x}}=\Sigma_{\boldsymbol{x}}$ dans $\Omega_{\boldsymbol{x}}$ pour presque tous les $\boldsymbol{x}$, alors $T=\Sigma$ dans $G$ (et vice versa). Si la section $T_{\boldsymbol{x}}$ sur $\Omega_{\boldsymbol{x}}$ existe pour chaque $\boldsymbol{x}$ et si elle est une fonction distributionnelle localement sommable dans $G$, alors $T$ coüncide avec la distribution définie par $T_{\boldsymbol{x}}$ dans $G$.

CoRollame 2. Si $T_{x} \geqslant 0$ dans $\Omega_{x}$ pour presque tous les $x$, alors $T \geqslant 0$ dans $G$; si $T_{\boldsymbol{x}} \geqslant S_{\boldsymbol{x}}$ dans $\Omega_{\boldsymbol{x}}$ pour presque tous les $\boldsymbol{x}$, alors $T \geqslant S$ dans $G$.

CoRollatRe 3. Si $T_{\boldsymbol{x}}=0$ dans $\Omega_{\boldsymbol{x}}$ pour presque tous les $\boldsymbol{x}$, alors $T=0$ dans $G ;$ si $T_{x}=S_{x}$ dans $\Omega_{x}$ pour presque tous les $x$, alors $T=S$ dans G. La distribution dans laquelle on peut fixer $\boldsymbol{x}=\boldsymbol{x}_{0}$ pour tout $\boldsymbol{x}_{0}$ est complètement déterminée par ses sections.

\section{§ 6. Autres espèces de convergence dans les définitions du \$.2}

Dans ce $\S$ nous nous occuperons de la limite

$$
\lim \boldsymbol{T}\left(\boldsymbol{x}_{0}+\boldsymbol{A} \boldsymbol{x}+\boldsymbol{s}, \boldsymbol{y}\right) \quad(\operatorname{det} \boldsymbol{A} \neq 0),
$$

où $|\boldsymbol{A}| \rightarrow 0$ et $|\boldsymbol{s}| \rightarrow 0$ d'une certaine manière. Dans les $\mathrm{N}^{0} 6.1$ et 6.2 . les théorèmes ne sont établis que pour la fixation et pour la valeur, car dans le cas de la limite les raisonnements sont analogues.

6.1. La limite $(6.0)$, où $|\boldsymbol{A}|^{m}=O(\operatorname{det} \boldsymbol{A})$ et $|\boldsymbol{s}|=O(|\boldsymbol{A}|)$. Dans ce cas nous montrerons que l'existence da la limite (6.0) équivaut à celle de la section $T\left(\boldsymbol{x}_{0}, \boldsymbol{y}\right)$.

THÉORÈME 1. Si $T\left(\boldsymbol{x}_{0}, \boldsymbol{y}\right)=S(\boldsymbol{y})$ dans un ouvert $\Omega \subset\left(\mathcal{E}^{n}\right)_{\boldsymbol{y}}$, alors (6.1.1) $\lim T\left(\boldsymbol{x}_{0}+\boldsymbol{A} \boldsymbol{x}+\boldsymbol{s}, \boldsymbol{y}\right)=S(\boldsymbol{y}) \quad$ lorsque $\quad|\boldsymbol{A}|+|\boldsymbol{s}| \rightarrow 0$, de façon que $|\boldsymbol{A}|^{m}=O(\operatorname{det} \boldsymbol{A})$ et $|\boldsymbol{s}|=O(|\boldsymbol{A}|)$, dans un voisinage de l'ensemble $\left\{\boldsymbol{x}_{0}\right\} \times \Omega$.

Démonstration. On peut admettre $x_{0}=0$ et $S(\boldsymbol{y})=0$. Soit $\chi \in \mathcal{D}_{\left(\varepsilon^{m}\right)_{x} \times \Omega}$ et supposons que $\lambda=|\boldsymbol{A}| \rightarrow 0$ de façon que $\lambda^{m}=O(\operatorname{det} \boldsymbol{A})$ et $|s|=O(\lambda)$. Comme on a

$$
\left|\boldsymbol{A}^{-1}\right|=O\left(\frac{\lambda^{m-1}}{|\operatorname{det} \boldsymbol{A}|}\right)
$$

les quantités $\left|\lambda \boldsymbol{A}^{-1}\right|$ et $\left|\boldsymbol{A}^{-1} \boldsymbol{s}\right|$ restent bornées. Il en résulte que $\left(T\left(\boldsymbol{x}_{0}+\boldsymbol{A} \boldsymbol{x}+\boldsymbol{s}, \boldsymbol{y}\right), \chi(\boldsymbol{x}, \boldsymbol{y})\right)=\left(T\left(\boldsymbol{x}_{0}+\lambda \boldsymbol{x}, \boldsymbol{y}\right), \frac{\lambda^{m}}{|\operatorname{det} \boldsymbol{A}|} \chi\left(\lambda \boldsymbol{A}^{-1} \boldsymbol{x}-\boldsymbol{A}^{-1} \boldsymbol{s}, \boldsymbol{y}\right)\right) \rightarrow 0$, c. q. f. d.

LEMCME. Si $S(t, x)$ est une distribution dans un ouvert $G \subset(\mathcal{E})_{t} \times\left(\mathcal{E}^{n}\right)_{\boldsymbol{y}}$ telle que $t S(t, \boldsymbol{y})=0$ dans $G$ et $S(t, \boldsymbol{y})=S(-t, \boldsymbol{y})$ dans un voisinage de tout point $(0, \boldsymbol{y}) \epsilon G$, alors $S=0$ dans $G$.

Démonstration. Soit $V$ un voisinage symétrique d'un point $(0, \boldsymbol{y}) \epsilon G$ dans lequel $S(t, y)+S(-t, y)=0$ et soit $\varphi \epsilon \mathscr{D}_{V}$. Il suffit de prouver que $(S, \varphi)=0$. Or on a

$$
\varphi(t, \boldsymbol{y})=\frac{\varphi(t, \boldsymbol{y})+\varphi(-t, \boldsymbol{y})}{2}+t \psi(t, \boldsymbol{y}) \quad \text { où } \quad \psi \in \mathcal{D}_{V},
$$

ce qui donne

$$
(S, \varphi)=\frac{1}{2}(S(t, \boldsymbol{y})+S(-t, \boldsymbol{y}), \varphi(t, \boldsymbol{y}))+(t S(t, \boldsymbol{y}), \psi(t, \boldsymbol{y}))=0 .
$$

THÉORÈme 2 (Z. Zieleźny) ${ }^{17}$ ). Si la limite

$$
\lim T\left(\boldsymbol{x}_{0}+\boldsymbol{A} \boldsymbol{x}, \boldsymbol{y}\right)
$$

existe lorsque $|\boldsymbol{A}| \rightarrow 0$ de façon que $|\boldsymbol{A}|^{m}=O(\operatorname{det} \boldsymbol{A})$, alors elle ne dépend pas de $\boldsymbol{y}$.

\footnotetext{
${ }^{17}$ ) Cf. [9] pour le cas d'une variable $(m=1, n=0)$.
} 
Démonstration. On peut admettre $x_{0}=0$. Désignons par $T_{0}(\boldsymbol{x}, \boldsymbol{y})$ la limite en question. Si $c_{i} \neq 0(i=1, \ldots, m)$, on a en particulier

$$
T\left(\lambda \frac{x_{1}}{c_{1}}, \ldots, \lambda \frac{x_{m}}{e_{m}}, \boldsymbol{y}\right) \rightarrow T_{0}(\boldsymbol{x}, \boldsymbol{y}) \quad \text { pour } \quad \lambda \rightarrow 0,
$$

d'où $T\left(\lambda x_{1}, \ldots, \lambda x_{m}, \boldsymbol{y}\right) \rightarrow T_{0}\left(c_{1} x_{1}, \ldots, c_{m} x_{m}, \boldsymbol{y}\right)$. Il en résulte que $\boldsymbol{T}_{0}\left(c_{1} x_{1}, \ldots, c_{m} x_{m}, \boldsymbol{y}\right)=T_{0}\left(x_{1}, \ldots, x_{m}, \boldsymbol{y}\right)$.

En dérivant par rapport à $\left.c_{1}{ }^{18}\right)$ et en posant $c_{i}=1(i=1, \ldots, m)$, on obtient

$$
x_{1} \frac{\partial T_{0}}{\partial x_{1}}\left(x_{1}, \ldots, x_{m}, \boldsymbol{y}\right)=0
$$

on a, de plus,

$$
\frac{\partial T_{0}}{\partial x_{1}}\left(x_{1}, \ldots, x_{m}, \boldsymbol{y}\right)+\frac{\partial T_{0}}{\partial x}\left(-x_{1}, \ldots, x_{m}, \boldsymbol{y}\right)=0,
$$

car $T_{0}\left(-x_{1}, x_{2}, \ldots, x_{m}, y\right)=T_{0}\left(x_{1}, x_{2}, \ldots, x_{m}, \boldsymbol{y}\right)$, donc, d'après le lemme, $\partial T_{0} / \partial x_{1}=0$. Pareillement $\partial T_{0} / \partial x_{i}=0(i=2, \ldots, m)$, ce qui montre que $T_{0}$ ne dépend pas de $x$.

Les théorèmes 1 et 2 entraînent le

CoRollatre 1. L'existence de la limite (6.1.2) est une condition nécessaire et suffisante pour qu'on puisse fixer $x=x_{0}$ dans $T$.

Finalement le théorème $\mathrm{du} \mathrm{N}^{0} 4.5$ donne:

CoRollamRe 2. Pour que la section $T\left(x_{0}, y\right)$ existe sur un ouvert $\Omega \subset\left(\mathcal{E}^{n}\right)_{y}$ il faut et il suffit que la limite

$$
\lim \left(T\left(\boldsymbol{x}_{0}+\boldsymbol{A} \boldsymbol{x}, \boldsymbol{y}\right), \varphi(\boldsymbol{x}) \psi(\boldsymbol{y})\right)
$$

existe lorsque $|\boldsymbol{A}| \rightarrow 0$ de façon que $|\boldsymbol{A}|^{m}=0(\operatorname{det} \boldsymbol{A})$, pour $\psi \epsilon \mathcal{D}_{\Omega}$ et $\phi \epsilon,(\mathscr{D})_{x}$.

En effet, si l'on pose $U_{\psi}=(T, \psi)_{y}$, on trouve $\left(U_{\psi}\left(\boldsymbol{x}_{0}+\boldsymbol{A} \boldsymbol{x}\right), \varphi\right)_{x}$ $=\left(\boldsymbol{T}\left(\boldsymbol{x}_{0}+\boldsymbol{A} \boldsymbol{x}, \boldsymbol{y}\right), \varphi(\boldsymbol{x}) \psi(\boldsymbol{y})\right)_{\boldsymbol{x}, \boldsymbol{y}}$. Par conséquent, en vertu du corollaire 1. (pour le cas $n=0$ ), l'existence de la limite (6.1.3) entraîne celle de la valeur $U_{\psi}\left(x_{0}\right)$, d'où il résulte, selon le théorème $d u N^{0} 4.5$, qu'on peut fixer $\boldsymbol{x}=\boldsymbol{x}_{0}$ sur $\Omega$.

Remarque. L'hypothèse sur l'existence de la limite $\lim T\left(\boldsymbol{x}_{0}+\lambda \boldsymbol{x}, \boldsymbol{y}\right)$ n'entraîne que l'homogénéité d'ordre 0 par rapport à $\boldsymbol{x}$ de cette limite (c'est-à-dire, p. ex., que si l'on passe aux coordonnées sphériques $\boldsymbol{x}=\boldsymbol{x}(r, \boldsymbol{\vartheta})$ cette limite ne dépend pas de $r$ ). Par contre, il suffit de supposer (dans le

18) On peut prendre le produit sealaire. théorème 2) que la limite

$$
\lim T\left(x_{10}+\lambda_{1} x_{1}, \ldots, x_{m 0}+\lambda_{m} x_{m}, \boldsymbol{y}\right)
$$

existe lorsque $\lambda_{1} \rightarrow 0, \ldots, \lambda_{m} \rightarrow 0$ de façon que $\lambda_{i} / \lambda_{j}<1+\varepsilon$ (pour un $\varepsilon>0$ ).

6.2. Omission de la condition $|\boldsymbol{s}|=O(|\boldsymbol{A}|)$. Soit $\varphi(\boldsymbol{x})$ une fonction non négative de $(\mathcal{D})_{x}$ telle que $\int \varphi d x=1$. Du lemme du $\mathrm{N}^{0} 5.1$ on tire le

LEMME. Soit $T$ une distribution dans un ouvert $G \subset\left(\mathcal{E}^{m}\right)_{x}$. Si $\left(T, \varphi_{\lambda}(\boldsymbol{x}-\xi)\right) \geqslant a$ (resp. $\left.\leqslant a\right)$ pour $\xi \epsilon G$ et pour $\lambda$ suffisamment petit $(0<\lambda<\lambda(\xi))$, alors $T \geqslant a$ (resp. $T \leqslant a)$ dans $G$.

THÉORÈME 1. Si la limite

$$
\lim _{\lambda \rightarrow 0+, s \rightarrow 0} T\left(x_{0}+\lambda x+s\right)
$$

existe (dans un voisinage de $\boldsymbol{x}_{0}$ ), alors $T$ est une fonction dans un voisinage de $\boldsymbol{x}_{0}$, continue au point $\boldsymbol{x}_{0}{ }^{19}$ ) et vice versa.

Démonstration. Soient $\varphi$ et $\varphi_{\lambda}$ comme dans le lemme. D'après les hypothèses la limite

$$
\lim _{\lambda \rightarrow 0+, s \rightarrow 0}\left(T\left(x_{0}+\lambda \boldsymbol{x}+\boldsymbol{s}\right), \varphi\right)=\lim _{\lambda \rightarrow 0+, \xi \rightarrow x_{0}}\left(T, \varphi_{\lambda}(\boldsymbol{x}-\xi)\right)=C
$$

existe. Il en résulte qu'à tout $\varepsilon>0$ correspond un $\delta(\varepsilon)>0$ tel que $C-\varepsilon<\left(T, \varphi_{\lambda}(\boldsymbol{x}-\xi)\right)<C+\varepsilon$ lorsque $0<\lambda<\delta(\varepsilon)$ et $\left|\boldsymbol{\xi}-\boldsymbol{x}_{0}\right|<\delta(\varepsilon)$. Selon le lemme on a donc $C-\varepsilon \leqslant T \leqslant C+\varepsilon$ pour $\left|\boldsymbol{x}-\boldsymbol{x}_{0}\right|<\delta(\varepsilon)$, ce qui montre que $T$ est une fonction dans $\left|\boldsymbol{x}-\boldsymbol{x}_{0}\right|<\delta(1)$, continue au point $\boldsymbol{x}_{0}$.

THÉORÈmE 2. Soit $\Omega$ un ouvert de $\left(\varepsilon^{n}\right)_{y}$. Si la limite

$$
\lim _{\lambda \rightarrow 0+, s \rightarrow 0} T\left(x_{0}+\lambda x+s, y\right)
$$

existe dans un ouvert qui contient l'ensemble $\left\{x_{0}\right\} \times \Omega$, alors la distribution $T$ est de la forme

$$
\quad T(\boldsymbol{x}, \boldsymbol{y})=S_{\boldsymbol{x}}(\boldsymbol{y})
$$

dans un ouvert qui contient l'ensemble $\left\{x_{0}\right\} \times \Omega$, où $S_{x}$ est une fonction distributionnelle continue au point $\boldsymbol{x}_{0}$. Réciproquement, si $T$ est de la forme (6.2.2), la limite (6.2.1) existe.

Démonstration. On vérifie que le théorème réciproque est vrai: si $\varphi \in \mathcal{D}_{\left(\varepsilon^{m}\right)_{\boldsymbol{x}} \times \Omega}$, alors

$\left(T\left(\boldsymbol{x}_{0}+\lambda \boldsymbol{x}, \boldsymbol{y}\right), \varphi(\boldsymbol{x}, \boldsymbol{y})\right)=\int\left(S_{\boldsymbol{x}_{0}+\lambda \boldsymbol{x}+\boldsymbol{s}}, \varphi(\boldsymbol{x}, \boldsymbol{y})\right)_{\boldsymbol{y}} d \boldsymbol{x} \rightarrow \int\left(S_{\boldsymbol{x}_{0}}, \varphi(\boldsymbol{x}, \boldsymbol{y})\right)_{\boldsymbol{y}} d \boldsymbol{x}$

19) Il suffit de supposer la convergence de $\left(T\left(x_{0}+\lambda x+s\right), \varphi\right)$ pour une seule fonction non négative $\varphi \in D$ qui ne s'annule pas identiquement. 
pour $\lambda \rightarrow 0$, puisque $\left(S_{\boldsymbol{x}_{0}+\lambda \boldsymbol{x}+\boldsymbol{s}}, \varphi(\boldsymbol{x}, \boldsymbol{y})\right)_{\boldsymbol{y}} \rightarrow\left(S_{\boldsymbol{x}}, \varphi(\boldsymbol{x}, \boldsymbol{y})\right)_{\boldsymbol{y}}$ uniformément par rapport à $\boldsymbol{x}$.

Supposons maintenant que la limite (6.2.1) existe. Soit $\Omega_{0}$ un ouvert borné tel que $\bar{\Omega}_{0} \subset \Omega$; il suffit de prouver que $T$ est de la forme (6.2.2) dans $\Delta \times \Omega_{0}$, où $\Delta$ est un voisinage de $\boldsymbol{x}_{0}$. Soient $\varphi$ et $\varphi_{\lambda}$ comme dans le lemme; donc la limite

$$
\lim _{\lambda \rightarrow 0+, \xi \rightarrow x_{0}}\left(T, \varphi_{\lambda}(\boldsymbol{x}-\boldsymbol{\xi}) \psi(\boldsymbol{y})\right)
$$

existe pour tout $\psi \epsilon \mathcal{D}_{\Omega}$. Posons

$$
S_{\lambda, \boldsymbol{\xi}}(\boldsymbol{y})=\left(T(\boldsymbol{x}, \boldsymbol{y}), \varphi_{\lambda}(\boldsymbol{x}-\boldsymbol{\xi})\right)_{\boldsymbol{x}} \quad \text { et } \quad S_{0}=\lim _{\lambda \rightarrow 0+, \xi \rightarrow x_{0}} S_{\lambda, \xi} \quad \text { dans } \Omega
$$

(pour tout ouvert borné $\Omega_{1}$ tel que $\bar{\Omega}_{1} \subset \Omega, S_{\lambda, \xi}$ sont définies dans $\Omega_{2}$ pourvu que $\lambda$ et $\left|\xi-x_{0}\right|$ soient suffisamment petits). Il existe un $k \epsilon \mathcal{Z}_{0}$ tel que $\left(S_{0}, \psi\right)_{y}$ est borné et $\left(S_{\lambda, \xi}, \psi\right)_{y}$ converge uniformément vers $\left(S_{0}, \psi\right)$ pour $\psi \epsilon \mathcal{D}_{\Omega_{0}},\|\psi\|_{k} \leqslant 1$. Alors à chaque $0<\varepsilon<1$ correspond un $\delta(\varepsilon)>0$ tel que

(6.2.3) $-M<\left(S_{0}, \psi\right)-\varepsilon<\left(S_{\lambda, \xi}, \psi\right)=\left(T, \varphi_{\lambda}(\boldsymbol{x}-\xi) \psi(y)\right)<\left(S_{0}, \psi\right)+\varepsilon<M$ lorsque $\psi \in \mathcal{D}_{\Omega_{0}},\|\psi\|_{k} \leqslant 1,0<\lambda<\delta(\varepsilon),\left|\xi-x_{0}\right|<\delta(\varepsilon)$

où $M$ est une constante. Si l'on pose $T_{w}=(T(\boldsymbol{x}, \boldsymbol{y}), \psi(\boldsymbol{y}))_{\boldsymbol{y}}$, on trouve $-M<\left(T_{\psi}, \varphi_{\lambda}(\boldsymbol{x}-\boldsymbol{\xi})\right)<M$; il en résulte, d'après le lemme, que $T_{p}$ est une fonction $f_{\psi}(\boldsymbol{x})$ bornée dans $\left|\boldsymbol{x}-\boldsymbol{x}_{0}\right|<\delta(1)$ pour tout $\left.\psi \epsilon^{\prime}\right)_{\Omega_{0}}$ :

$$
f_{\psi}(\boldsymbol{x})=\left(T,\left.\psi(\boldsymbol{y})\right|_{\boldsymbol{y}} .\right.
$$

D'autre part, d'après (6.2.3), $\left(S_{\lambda, \xi}, \psi\right)$ sont équicontinues par rapport à $\psi$ (dans $\mathcal{D}_{\Omega_{0}}$ avec la norme \|\|$_{k}$ ), donc pour tout $\xi$ tel que $0<\left|\xi-x_{0}\right|$ $<\delta(1)$ il existe une suite $\lambda_{v} \rightarrow 0$ telle que $\left(S_{\lambda_{p}, \xi}, \psi\right)$ converge dans $D_{D_{0}}$; la limite $S_{\xi}=\lim _{v \rightarrow \infty} S_{\lambda_{p}, \xi}$ satisfait donc à la condition

$$
\left(S_{0}, \psi\right)-\varepsilon \leqslant\left(S_{\xi}, \psi\right) \leqslant\left(S_{0}, \psi\right)+\varepsilon
$$

pour $\psi \in \mathscr{D}_{\Omega_{0}},\|\psi\|_{k} \leqslant 1,\left|\xi-x_{0}\right|<\delta(\varepsilon)$, en vertu de (6.2.3). II on résulte que si l'on pose $S_{x_{0}}=S_{0}$ (dans $\Omega_{0}$ ), la fonction distributionnelle $S_{\infty}$ est continue au point $\boldsymbol{x}_{0}$.

Selon le corollaire 2 du $N^{0} 4.6$ la fonction $f_{p}(x)$ possède une valeur (au sens des distributions) en $\xi$ égale à $f_{p}(\xi)$ presque partout. In vertu de $(6.2 .3)$ et (6.2.4) nous avons, pour un tel $\xi,\left(S_{\lambda, \xi}, \psi\right)_{y}=\left(f_{p}, \varphi_{\lambda}\right)_{x \rightarrow} \rightarrow f_{p}(\xi)$. On a donc (selon la définition de $S_{\xi}$ ):

(6.2.5) $\quad\left(S_{\xi}, \psi\right)=\int_{\psi}(\xi)$ presque partout pour $\left|\xi-x_{0}\right|<\delta(1)$.
La fonction distributionnelle $S_{x}$ est donc sommable. Soit maintenant $\alpha(\boldsymbol{x})$ une fonction de $(\mathcal{D})_{\boldsymbol{x}}$ de support contenu dans $\left|\boldsymbol{x}-\boldsymbol{x}_{0}\right|<\delta(1)$ et soit $\psi \epsilon \mathcal{D}_{\Omega_{0}}$. On a, d'après $(6.2 .4)$ et $(6.2 .5)$,

$$
(T, \alpha(\boldsymbol{x}) \psi(\boldsymbol{y}))=\int f_{\psi}(\boldsymbol{x}) \alpha(\boldsymbol{x}) d \boldsymbol{x}=\int\left(\mathcal{S}_{\boldsymbol{x}}, \alpha(\boldsymbol{x}) \psi(\boldsymbol{y})\right)_{\boldsymbol{y}} d \boldsymbol{x} .
$$

Il en résulte que $T=S_{\boldsymbol{x}}(\boldsymbol{y})$ pour $\left|\boldsymbol{x}-\boldsymbol{x}_{0}\right|<\delta(1)$ et $\boldsymbol{y} \in \Omega_{0}$, ce qui termine la démonstration.

6.3. Omission de la condition $|\boldsymbol{A}|^{m}=O(\operatorname{det} \boldsymbol{A})$, Dans ce cas on obtient aussi une condition strictement plus forte, comme le montre l'exemple $d$. la distribution $T=x^{2} \delta(y)$ : on a

$$
\lim _{\lambda \rightarrow 0+} T(\lambda x, \lambda y)=\lim _{\lambda \rightarrow 0+} \lambda x^{2} \delta(y)=0,
$$

tandis que $T(\lambda x, \mu y)=\lambda^{2} \mu^{-1} x^{2} \delta(y)$ ne converge pas pour $\lambda \rightarrow 0, \mu \rightarrow 0$.

LEMVE. Soit $T(\boldsymbol{x}, y)$ (on suppose $n=1)^{20}$ ) une distribution définie dans $G \times(0, \eta)$, ò̀ $\eta>0$ et $G$ soit un ouvert de $\left(\mathcal{E}^{m}\right)_{x}$. Si

$$
\lim _{\substack{0<\lambda<\mu \rightarrow 0 \\ 1 \xi \xi \epsilon_{0}}} T(\xi+\lambda \boldsymbol{x}, \mu y)=0,
$$

oì $G_{0}$ est un ouvert borné, $\bar{G}_{0} \subset G$ (il s'agit de la limite suivant le filtre de base $\left.\left.U_{\nu}=\left((\lambda, \mu, \xi): 0<\lambda<\mu<1 / v, \xi^{\prime \prime} \in G_{0}\right)\right)^{21}\right)$, alors la distribution $T$ est de la forme

$$
\quad T(\boldsymbol{x}, y)=S_{\boldsymbol{x}}(y)
$$

dans un voisinage de $G_{0} \times\{0\}$ pour $y>0$, où $S_{x}$ est une fonction distributionnelle localement sommable et telle que $\lim S_{x}(y)=0$ uniformément pour

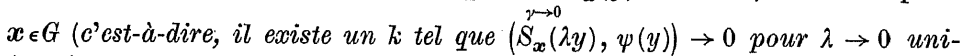
formément par rapport à $\left.\boldsymbol{x} \in G_{0}, \psi \in \mathbb{D}_{(0,1)},\|\psi\|_{k} \leqslant 1\right)$. Le théorème réciproque est aussi vrai.

La démonstration est analogue à celle du théorème $2 \mathrm{du} \mathrm{X}^{0} 6.2$. On vérifie sans peine que le théorème réciproque est vrai. Supposons que (6.3.1) ait lieu. Soient $\varphi$ et $\varphi_{\lambda}$ comme dans le lemme dú № 6.2. Il existe donc un $k \epsilon^{\top} \mathcal{Z}_{0}$ tel que $(T(\xi+\lambda \boldsymbol{x}, \mu y), \varphi(\boldsymbol{x}) \psi(y)) \rightarrow 0$ pour $0<\lambda<\mu \rightarrow 0$,

20) Le lemme reste vrai (et la démonstration est analogue) pour $n>1$.

21) On voit à l'exemple de la distribution

$$
T=\sum_{n \mid 1}^{\infty} \frac{1}{3^{n}} \delta\left(x-\frac{1}{2^{n}}, y-\frac{1}{2^{n}}\right)
$$

qu'il ne suffit pas de supposer qu'on ait $\lim _{0<\lambda<\mu \rightarrow 0} T(\xi+\lambda x, \mu y)=0$ pour tout $\xi \in G$. 
uniformément par rapport à $\xi \epsilon G_{0}$ et $\psi \epsilon^{(\mathcal{D})}(0,1),\|\psi\|_{k} \leqslant 1$. Alors à tout $\varepsilon>0$ il correspond un $\delta(\varepsilon)>0$ tel que

$$
-\varepsilon \leqslant\left(S_{\lambda, \xi}, \frac{1}{\mu} \psi\left(\frac{y}{\mu}\right)\right)=\left(T, \varphi_{\lambda}(x-\xi) \frac{1}{\mu} \psi\left(\frac{y}{\mu}\right)\right) \leqslant \varepsilon
$$

pour $0<\lambda<\mu<\delta(\varepsilon), \xi \in G_{0}, \psi \in \mathscr{D}_{(0,1)},\|\psi\|_{k} \leqslant 1$, où

$$
S_{\lambda, \xi}(y)=\left(T(x, y), \varphi_{\lambda}(\boldsymbol{x}-\xi)\right)_{\boldsymbol{x}} .
$$

Comme dans la démonstration du théorème $2 \mathrm{du} \mathrm{N}^{0} 6.2$, on définit les fonctions $f_{\psi}(x)=(T(x, y), \psi(y))_{\nu}$, les distributions $S_{\xi}=\lim _{y \rightarrow \infty} S_{\nu, \xi}$, où $\lambda_{\text {est }}$ une suite convergente vers 0 qui dépend de $\xi$, et on montre que $T$ est de la forme (6.3.2). Enfin l'inégalité (6.3.3) entraîne

$$
-\varepsilon \leqslant\left(S_{\xi}, \frac{1}{\mu} \psi\left(\frac{y}{\mu}\right)\right) \leqslant \varepsilon
$$

pour $0<\mu<\delta(\varepsilon), \xi \in G_{0}, \psi \epsilon \mathcal{D}_{(0,1)},\|\psi\|_{k} \leqslant 1$. Cela montre que $\lim _{y \rightarrow 0} S_{x}(y)=0$ uniformément pour $\boldsymbol{x} \epsilon G_{0}$.

THÉORÈnE. Pour que la limite

$$
\lim _{|\boldsymbol{A}| \rightarrow 0} T\left(\boldsymbol{x}_{0}+\boldsymbol{A} \boldsymbol{x}\right)=C \quad(\operatorname{det} \boldsymbol{A} \neq 0)
$$

existe dans un voisinage de $\boldsymbol{x}_{0}$ pour $\boldsymbol{x} \neq \boldsymbol{x}_{0}$, il faut et il suffit que la distribution $T$ soit de la forme

$$
\left.(T, \varphi)=\int\left(S_{\omega}(r), r^{m-1} \varphi\right)_{r} d_{\omega \omega}{ }^{22}\right)
$$

dans un voisinage de $\boldsymbol{x}_{0}$ pour $\boldsymbol{x} \neq \boldsymbol{x}_{0}$ (l'intégrale étant étendue sur la sphère $\left.r=\left|\boldsymbol{x}-\boldsymbol{x}_{0}\right|=1\right)$, oì $\boldsymbol{S}_{\omega}(r)$ est une fonction distributionnelle localement sommable telle que $\lim S_{\omega}(r)=C$ uniformément par rapport à ¿ (c'est-ḋ-dire il existe un $k$ tel que $\left(S_{\omega}(\lambda r)-C, \psi(r)\right) \rightarrow 0$ pour $\lambda \rightarrow 0$, uniformément par rapport d̀ $\omega$ et $\left.\psi \in \mathbb{D}_{(0,1)},\|\psi\|_{k} \leqslant 1\right)$.

Démonstration. On peut admettre $x_{0}=\mathbf{0}$ et $\theta=0$. On vérifie que la condition est suffisante. Pour montrer qu'elle est nécessaire, il suffit de prouver que (6.3.5) a lieu dans un voisinage de $\mathbf{0}$ pour $x_{m}>0$, $\left|x_{i}\right| x_{m} \mid<2(i=1, \ldots, m-1)$; en effet, on peut ensuite faire un recollement, en vertu de l'unicité de la représentation (6.3.5) (ef. № 1.4).

22) On peut dire que $T$ est une fonction de $\boldsymbol{~}$.
Soit

$$
\boldsymbol{A}=\left[\begin{array}{cccc}
\lambda \mu & \ldots & 0 & \xi_{1} \mu \\
\ldots & \ldots & \ldots & \ldots \\
0 & \ldots & \lambda \mu & \xi_{m-1} \mu \\
0 & \ldots & 0 & \mu
\end{array}\right]
$$

Supposons que $\lambda \rightarrow 0+, \mu \rightarrow 0+$ et $\left|\xi_{i}\right| \leqslant 3$; on a alors $\boldsymbol{A} \rightarrow \mathbf{0}$ et $\operatorname{det} \boldsymbol{A} \neq \mathbf{0}$. Nous avons donc, d'après l'hypothèse (6.3.4),

$$
\boldsymbol{T}(\boldsymbol{A x})=T\left(\lambda \mu x_{1}+\mu \xi_{1} x_{m}, \ldots, \lambda \mu x_{m-1}+\mu \xi_{m-1} x_{m}, \mu x_{m}\right) \rightarrow 0
$$

dans un voisinage de $\mathbf{0}$ pour $\boldsymbol{x} \neq \mathbf{0}$. Si l'on pose

$$
\Sigma\left(u_{1}, \ldots, u_{m-1}, t\right)=T\left(t u_{1}, \ldots, t u_{m-1}, t\right)
$$

pour $0<t<\delta_{1}$ et $\left|u_{i}\right|<3(i=1, \ldots, m-1)$, on trouve

$$
\begin{aligned}
\Sigma\left(\xi_{1}+\lambda u_{1}, \ldots, \xi_{m-1}\right. & \left.+\lambda u_{m-1}, \mu t\right) \\
& \neq T\left(\lambda \mu u_{1} t+\mu \xi_{1} t, \ldots, \lambda \mu u_{m-1} t+\mu \xi_{m-1} t, \mu t\right) \rightarrow 0 .
\end{aligned}
$$

$\mathrm{Il}$ en résulte, selon le lemme, que

$$
\Sigma(\boldsymbol{u}, t)=\bar{S}_{\boldsymbol{u}}(t) \quad \text { pour } \quad 0<t<\delta_{2} \text { et pour }\left|u_{i}\right|<2
$$

$$
(i=1, \ldots, m-1),
$$

où $\lim \bar{S}_{\boldsymbol{u}}(t)=0$ uniformément pour $\left|u_{i}\right|<2(i=1, \ldots, m-1)$. Si $\varphi \epsilon(\mathcal{D})_{\boldsymbol{x}}$ - est une fonction de support contenu dans l'ensemble $0<x_{m}<\delta_{2}$, $\left|x_{i}\right| x_{m} \mid<2$, alors d'après $(6.3 .6)$ et $(6.3 .7)$, on a

$$
(T, \varphi)=\left(\Sigma, t^{m-1} \varphi\left(t u_{1}, \ldots, t u_{m-1}, t\right)\right)=\int\left(\bar{S}_{u t}(t), \varphi\left(t u_{1}, \ldots, t u_{m-1}, t\right)\right)_{t} d u .
$$

En passant aux coordonnées sphériques $\left(r=t \sqrt{1+u_{1}^{2}+\ldots+u_{m-1}^{2}}\right)$ et en posant $S_{\omega}(r)=\left(1+u_{1}^{2}+\ldots+u_{m-1}^{2}\right)^{-m / 2} \bar{S}_{u}(t)$ nous obtenons $(6.3 .5)$.

Remarque 1. Si la limite (6.3.4) existe dans un voisinage de $x_{0}$ (ce qui entraîne que la valeur $T\left(\boldsymbol{x}_{0}\right)$ existe), on a

$$
(T, \varphi)=\int\left\{\int_{0}^{\infty} S_{\boldsymbol{\omega}}(r) r^{m-1} \varphi d r\right\} d \omega,
$$

où l'intégrale $\int_{0}^{\infty}$ doit être entendue au sens de la définition de $[5], \S 6^{23}$ ).

23) On le montre en faisant la décómposition $\varphi=\varphi(1-\alpha(r / \varepsilon))+\varphi \alpha(r / \varepsilon)$, où $\alpha \in D_{(-2,2)}, \alpha=1$ dans $[-1,1]$, et en faisant ensuite $\varepsilon \rightarrow 0$. 
Remarque 2. On a un théorème analogue dans le cas de la fixar tion des variables.

Remarque 3. L'existence de la limite (6.3.4) entraîne que $T$ ne possède de masse sur aucun rayon d'extrémité $x_{0}$ dans un voisinage de $\boldsymbol{x}_{0}$ (cf. le renvoi ${ }^{14}$ ) p. 30). En particulier, si $T$ est une mesure (pour $\boldsymbol{x} \neq \boldsymbol{x}_{0}$ ) elle ne peut avoir de masse en aucun point $\neq x_{0}$ d'un voisinage de $x_{0}$.

6.4. Valeur directionnelle. Nous verrons que la condition d'existence de la limite $\lim T(\lambda \boldsymbol{x}, \mu \boldsymbol{y})$ indique essentiellement les directions des hyperplans de $x^{\lambda, \mu \rightarrow 0+}$ $0<\lambda \leqslant \mu \rightarrow 0$ n'indique que celle de $x$

Nous dirons qu'une distribution $T(\boldsymbol{x}, \boldsymbol{y})$ possède une valeur diredionnelle par rapport à $\boldsymbol{y}$ en un point $\left(\boldsymbol{x}_{0}, \boldsymbol{y}_{0}\right)$, si la limite $\lim _{0<\lambda \leqslant \mu \rightarrow 0} T_{1}^{\prime}\left(\boldsymbol{x}_{0}+\lambda: \boldsymbol{x}, \boldsymbol{y}_{0}+\mu \boldsymbol{y}\right)$ existe dans un voisinage de $\left(\boldsymbol{x}_{0}, \boldsymbol{y}_{0}\right)$ et si elle est constante. La définition de la valeur directionnelle par rapport à la ,direction" d'un hyperplan $\pi$ passant par $\left(\boldsymbol{x}_{0}, \boldsymbol{y}_{0}\right)$ se ramène au cas précédent par l'introduction do coordonnées dans lequelles l'équation $x=x_{0}$ est celle de $\pi$. On montre que cette définition ne dépend pas du système de coordonnées. On définit pareillement la fixation des variables et la limite directionnelles.

Nous dirons qu'une distribution $T(\boldsymbol{x}, \boldsymbol{y})$ est bornée powr $\boldsymbol{x}=\boldsymbol{x}_{0}$ sur un ouvert $\Omega \subset\left(\mathcal{E}^{n}\right)_{y}$ si $T\left(\boldsymbol{x}_{0}+\lambda \boldsymbol{x}, \boldsymbol{y}\right)$ reste bornée lorsque $\lambda \rightarrow 0+$, pour $|x|<1$ et $\boldsymbol{y} \in \Omega$, c'est-à-dire

$$
\limsup _{\lambda \rightarrow 0+}\left|\left(T\left(\boldsymbol{x}_{0}+\lambda \boldsymbol{x}, \boldsymbol{y}\right), \varphi\right)\right|<\infty
$$

pour chaque $\varphi \in(D)_{x, y}$ de support contenu dans l'ensemble $\left.|\boldsymbol{x}|<1, \boldsymbol{y} \epsilon \Omega^{24}\right)$. On voit que si $T(\boldsymbol{x}, \boldsymbol{y})$ est bornée pour $x \rightarrow x_{0}$ sur $\Omega$, alors $T$ ne possède pas de masse sur $\boldsymbol{x}=\boldsymbol{x}_{0}, \boldsymbol{y} \in \Omega$ (cf. (4.4.1)).

Proposition 1. Si la valeur $T\left(\boldsymbol{x}_{0}, \boldsymbol{y}_{0}\right)$ directionnelle par rapport $\grave{a} \boldsymbol{y}$ existe, alors $T$ est bornée pour $\boldsymbol{x}=\boldsymbol{x}_{0}$ sur un voisinage de $\boldsymbol{y}_{0}$.

Démonstration. Par hypothèse, il existe un $M$, un $\delta>0$ et un $k \epsilon \chi_{0}$ tels que $\left|\left(T\left(\boldsymbol{x}_{0}+\lambda \boldsymbol{x}, \boldsymbol{y}_{0}+\mu \boldsymbol{y}\right), \chi(\boldsymbol{x}, \boldsymbol{y})\right)\right|<M$, lorsque $0<\lambda \leqslant \mu \leqslant \delta$, $\chi \in(D)_{x, y},\|\chi\|_{k} \leqslant 1$, le support de $\chi$ étant contenu dans l'ensemblo $|x|<1$, $|\boldsymbol{y}|<1$.

Soit maintenant $\varphi(\boldsymbol{x}, \boldsymbol{y})$ une fonction de $(\not D)_{x, y}$ de support contenu dans l'ensemble $|\boldsymbol{x}|<1,\left|\boldsymbol{y}-\boldsymbol{y}_{0}\right|<\delta$; la fonetion $\chi(\boldsymbol{x}, \boldsymbol{y}) \ldots \varepsilon\left(\boldsymbol{x}, \boldsymbol{y}_{0}+\delta \boldsymbol{y}\right)$ satisfait donc aux conditions ci-dessus ( $\varepsilon$ étant convenablement choisi),

24) Pareillement on dit que $T(x)$ est bornée en $x_{0}$ si $T\left(x_{11}+\lambda x\right)$ restí bornés pour $\lambda \rightarrow 0+$ dans un voisinage de $x_{0}$. d'où il résulte que

$$
\left.\mid T\left(\boldsymbol{x}_{0}+\lambda \boldsymbol{x}, \boldsymbol{y}\right), \varphi(\boldsymbol{x}, \boldsymbol{y})\right)\left|=\frac{\delta^{m}}{\varepsilon}\right|\left(T\left(\boldsymbol{x}_{0}+\lambda \boldsymbol{x}, \boldsymbol{y}_{0}+\delta \boldsymbol{y}\right), \chi(\boldsymbol{x}, \boldsymbol{y})\right) \mid \leqslant M \frac{\delta^{m}}{\varepsilon}
$$

pour $0<\lambda \leqslant \delta$. Ainsi $T$ est bornée pour $\boldsymbol{x}=\boldsymbol{x}_{0}$ sur $\left|\boldsymbol{y}-\boldsymbol{y}_{0}\right|<\delta$.

Cette proposition montre qu'une distribution $T(\boldsymbol{x}, \boldsymbol{y})$ qui possède en $\left(\boldsymbol{x}_{0}, \boldsymbol{y}_{0}\right)$ une valeur directionnelle par rapport à $\boldsymbol{y}$, ne possède pas de masse sur $\boldsymbol{x}=\boldsymbol{x}_{0}$ dans un voisinage de $\boldsymbol{y}_{0}$. Par contre, elle peut être une mesure dont le support est une droite arbitraire qui coupe l'hyperplan $\boldsymbol{x}=\boldsymbol{x}_{0}$ au point $\boldsymbol{x}_{0}$. On le voit (dans le cas $n=m=1$ ) à l'exemple de la mesure suivante, portée par la droite $y=a x$ :

$$
T=x^{2} \delta(y-\alpha x) \text {. }
$$
on a

$(T(\lambda x, \mu y), \varphi(x, y))=\int x^{2} \frac{1}{\lambda \mu} \varphi\left(\frac{x}{\lambda}, \frac{\alpha x}{\mu}\right) d x=\frac{\lambda^{2}}{\mu} \int x^{2} \varphi\left(x, \alpha \frac{\lambda}{\mu} x\right) d x \rightarrow 0$, lorsque $0<\lambda \leqslant \mu \rightarrow 0$.

Nous voyons donc que l'hyperplan $x=x_{0}$ est essentiellement distingué et que la condition d'existence de la valeur directionnelle est strictement plus forte que celle de la valeur et strictement plus faible que celle de la limite (6.3.4) (dans un voisinage), car celles-ci sont invariantes pour les rotations. Même la condition d'existence d'une valeur directionnelle pour chaque distribution qui résulte de $T$ par une substitution régulière n'est pas suffisante à ce que la limite (3.6.4) existe $\left.{ }^{25}\right)$. En effet, considérons la distribution $\left(n=m=1, x_{0}=y_{0}=0\right)$

$$
T=\sum_{\nu \mid 1}^{\infty} \frac{1}{10^{v}} \delta\left(x-\frac{1}{3^{v}}, y-\frac{1}{2^{v}}\right) \text {. }
$$

D'après la remarque $3 \mathrm{du} \mathrm{N}^{0} 6.3$, la limite (6.3.4) n'existe pas. Faisons dans $T(x, y)$ une substitution de classe $C^{2}$ (et de jacobien $\neq 0$ ), $u=u(x, y), v=v(x, y)$ telle que $u(0,0)=v(0,0)=0$; on obtient ainsi une distribution $\tilde{T}(u, v)$. Montrons que $\tilde{T}$ possède en $(0,0)$ une valeur directionnelle par rapport à $y$. On a

$$
\tilde{T}=\sum_{1}^{\infty} \frac{J_{v}}{10^{\prime \prime}} \delta\left(u-u_{v}, v-v_{v}\right)
$$

${ }^{25}$ ) Il semble probable que dans le cas d'une mesure $\mu$ il suffit de supposer l'existence d'une valeur directínnelle pour toute mesure qui résulte de $\mu$ par une substitution de classe $C^{1}$. 
oì $J_{v}$ est le jacobien pour $x=1 / 3^{v}, y=1 / 2^{p}$ et

$(6.4 .3)$

$$
\left\{\begin{array}{c}
u_{\nu}=u\left(\frac{1}{3^{v}}, \frac{1}{2^{\nu}}\right)=\frac{A}{3^{\nu}}+\frac{B}{2^{\nu}}+O\left(\frac{1}{4^{v}}\right), \\
v_{v}=v\left(\frac{1}{3^{\nu}}, \frac{1}{2^{\nu}}\right)=\frac{C}{3^{\nu}}+\frac{D}{2^{\nu}}+O\left(\frac{1}{4^{\nu}}\right), \\
A D-B C \neq 0 .
\end{array}\right.
$$

Soit $p \in(D)_{x, y}$; prenons un $l$ tel que le support de $\varphi$ soit contemu dans l'ensemble $|x|<1$. On a alors

$$
(\tilde{T}(\lambda x, \mu y), \varphi(x, y))=\left(\tilde{T}, \frac{1}{\lambda \mu} \varphi\left(\frac{x}{\lambda}, \frac{y}{\mu}\right)\right)=\frac{1}{\lambda \mu} \sum_{u_{\nu}<\lambda l}^{\Upsilon} \frac{J_{v}}{10^{\prime \prime}} \varphi\left(\begin{array}{cc}
u_{\nu} & v_{\nu} \\
\lambda & \mu
\end{array}\right) .
$$

En vertu de (6.4.3) l'inégalité $u_{v} \leqslant \lambda l$ entraîne $1 / 3^{v} \leqslant M \lambda$, où $M$ est une constante (indépendante de $\lambda$ ), donc

$$
\text { - }|(\tilde{T}(\lambda x, \mu y), \varphi(x, y))| \leqslant K \frac{1}{\lambda \mu} \sum_{\nu<\log _{3} M \lambda} \frac{1}{10^{\nu}} \leqslant \frac{L}{\lambda \mu} 10^{\log _{3} M \lambda}=\frac{L}{\lambda \mu}(M \lambda)^{\log _{3} 10}
$$

où $K, L$ sont des constantes (indépendantes de $\lambda$ et de $\mu$ ). II en résulte que $(\tilde{T}(\lambda x, \mu y), \varphi) \rightarrow 0$ lorsque $0<\lambda \leqslant \mu \rightarrow 0$, c. q. f. d.

On peut définir la valeur directionnelle par rapport à un système d'hyperplans $\pi_{1} \supset \pi_{2} \supset \ldots \supset \pi_{k}$ (passant par le point en question). Par exemple dans le cas $k=2$ on peut considérer la limite (constante)

$$
\lim _{0<\lambda \leqslant \mu \leqslant \sigma \rightarrow 0} T(\lambda \boldsymbol{x}, \mu \boldsymbol{y}, \sigma \boldsymbol{z})
$$

(s'il s'agit de la valeur directionnelle par rapport aux hyperplans de $(\boldsymbol{y}, \boldsymbol{z})$ et de $\boldsymbol{z})$. L'existence de cette limite entraîne celle de la valeur directionnelle par rapport à $(\boldsymbol{y}, \boldsymbol{z})$ et aussi par rapport à $\boldsymbol{z}$, mais l'assertion réciproque est fausse, comme le montre l'exemple de la distribution

$$
\text { (6.4.5) } \quad T=\sum_{1}^{\infty} \frac{1}{5^{p}} h\left(\frac{1}{4^{p}}+x\right) h\left(\frac{1}{4^{p}}-x\right) \delta\left(y-\frac{1}{3^{p}}\right) \delta\left(z-\frac{1}{2^{p}}\right)
$$

où $h(x)$ est la fonction d'Heaviside.

Des exemples montrent que l'existence d'une valeur dirxptioumelle par rapport à $(\boldsymbol{y}, \boldsymbol{z})$ n'entraîne pas celle par rapport à $\left.\boldsymbol{z}^{26}\right)$ et que l'existence

$$
\text { 26) } T=\sum_{1}^{\infty} \frac{1}{17^{\nu}} \delta\left(x-\frac{1}{4^{p}}\right) \delta\left(y-\frac{1}{3^{p}}\right) \delta\left(z-\frac{1}{2^{p}}\right) \text {. }
$$

d'une valeur directionnelle par rapport à toute direction parallèle a l'hyperplan $(\boldsymbol{y}, \boldsymbol{z})$ n'entraîne pas celle par rapport à $(\boldsymbol{y}, \boldsymbol{z})^{27}$ ).

On peut établir des théorèmes analogues à ceux du $\S 4$ et $d u \mathrm{~N}^{\circ} 6.1$ sur la valeur. Nous avons par exemple la

Propostrion 2. Si la valeur $T\left(\boldsymbol{x}_{0}, \boldsymbol{y}_{0}\right)=C$ directionnelle par rapport $\grave{a} \boldsymbol{y}$ existe, on a $\lim T\left(\boldsymbol{x}_{0}+\boldsymbol{A} \boldsymbol{x}+\boldsymbol{s}, \mu \boldsymbol{y}\right)=O$ lorsque $\mu \rightarrow 0+$, de façon que $|\boldsymbol{s}|=O(|\boldsymbol{A}|),|\boldsymbol{A}|=O(\mu)$ et $|\boldsymbol{A}|^{m}=O(\operatorname{det} \boldsymbol{A})$.

La démonstration est analogue à celle du théorème 1 du $\mathbb{N}^{\circ} 6.1$.

\subsection{Condition nécessaire et suffisante avec primitive pour la} valeur directionnelle. Nous avons le

THÉORغ̀me. Pour que la valeur $T\left(\boldsymbol{x}_{0}, \boldsymbol{y}_{\mathbf{0}}\right)$ directionnelle par rapport $\grave{a} y$ existe, il faut et il suffit qu'il existe un $(\boldsymbol{p}, \boldsymbol{q}) \mathcal{T}_{0}^{m+n}$ et une fonction $F^{\prime}(\boldsymbol{x}, \boldsymbol{y})$ continue dans un voisinage de $\left(\boldsymbol{x}_{0}, \boldsymbol{y}\right)$ tels que $D_{\boldsymbol{x}}^{\boldsymbol{p}} D_{\boldsymbol{y}}^{\boldsymbol{p}} F=T$ (dans un voisinage de $\left.\left(\boldsymbol{x}_{0}, \boldsymbol{y}_{0}\right)\right)$ et

(6.5.1) $F(\boldsymbol{x}, \boldsymbol{y})=C \frac{\left(\boldsymbol{x}-\boldsymbol{x}_{0}\right)^{\boldsymbol{p}}\left(\boldsymbol{y}-\boldsymbol{y}_{0}\right)^{\boldsymbol{q}}}{\boldsymbol{p} ! \boldsymbol{q} !}+o\left(\left|\boldsymbol{x}-\boldsymbol{x}_{0}\right|^{|\boldsymbol{p}|}\left(\left|\boldsymbol{x}-\boldsymbol{x}_{0}\right|+\left|\boldsymbol{y}-\boldsymbol{y}_{0}\right|\right)^{|\boldsymbol{\alpha}|}\right)$.

Démonstration. On peut admettre $C=0, x_{0}=0, y_{0}=0$.

$1^{\circ} \mathrm{La}$ condition est suffisante. Supposons que $T=D_{\boldsymbol{x}}^{\boldsymbol{p}} D_{\boldsymbol{y}}^{\boldsymbol{q}} W^{\prime}(\boldsymbol{x}, \boldsymbol{y})$ dans un voisinage de $(\boldsymbol{0}, \boldsymbol{0})$ et que $F^{\prime}(\boldsymbol{x}, \boldsymbol{y})=\varepsilon(\boldsymbol{x}, \boldsymbol{y})|\boldsymbol{x}|^{|\boldsymbol{p}|}(|\boldsymbol{x}|+|\boldsymbol{y}|)^{|\boldsymbol{\alpha}|}$, où $\varepsilon(\boldsymbol{x}, \boldsymbol{y}) \rightarrow \mathbf{0}$ pour $\boldsymbol{x} \rightarrow \mathbf{0}, \boldsymbol{y} \rightarrow \mathbf{0}$. On a alors

et

$$
T(\lambda \boldsymbol{x}, \mu \boldsymbol{y})=D_{\boldsymbol{x}}^{\boldsymbol{p}} D_{\boldsymbol{y}}^{\boldsymbol{\alpha}}\left(\frac{1}{\lambda^{|\boldsymbol{p}|} \mu^{|\boldsymbol{q}|}} F \cdot(\lambda \boldsymbol{x}, \mu \boldsymbol{y})\right)
$$

$$
\frac{1}{\lambda^{|\boldsymbol{p}|} \mu^{|\boldsymbol{\alpha}|}} F(\lambda \boldsymbol{x}, \mu \boldsymbol{y})=\varepsilon(\lambda \boldsymbol{x}, \mu \boldsymbol{y})|\boldsymbol{x}|^{|\boldsymbol{p}|}\left(\frac{\lambda}{\mu}|\boldsymbol{x}|+|\boldsymbol{y}|\right)^{|\boldsymbol{|}|} \rightarrow 0
$$

uniformément (dans tout ouvert borné de $(\boldsymbol{x}, \boldsymbol{y})$ ), lorsque $0<\lambda \leqslant \mu \rightarrow 0$, ce qui donne $T(\lambda \boldsymbol{x}, \mu \boldsymbol{y}) \rightarrow 0$ pour $0<\lambda \leqslant \mu \rightarrow 0$.

$2^{\circ}$ La condition est nécessaire $\left.{ }^{28}\right)$. Soient

(6.5.2) $\left\{\begin{array}{l}Q_{v \sigma} \text { l'intervalle }\left|x_{i}\right|<\lambda_{\nu},\left|y_{j}\right|<\lambda_{\sigma} \quad(i=1, \ldots, m ; j=1, \ldots, n), \\ Q_{\sigma}=Q_{\sigma \sigma}, \quad \lambda_{\nu}^{\prime}=1 / 2^{y} .\end{array}\right.$

27) $T=\sum_{1}^{\infty} \frac{1}{28^{p}} \delta\left(x-\frac{1}{3^{p}}\right) \delta\left(y-\frac{1}{2^{v}}\right) \delta(z)$.

28) Comme dans le No 4.1 il suffit de supposer que

$$
\lim _{\nu \geqslant \sigma \rightarrow \infty} T\left(x_{0}+\lambda_{\nu} x, y_{0}+\mu_{\sigma} y\right)=C
$$

pour un couple de suites $\left\{\lambda_{\nu}\right\},\left\{\mu_{\sigma}\right\}$ qui satisfont à la condition (4.1.1). 
Comme on a $T\left(\lambda_{\nu} \boldsymbol{x}, \lambda_{\sigma} \boldsymbol{y}\right) \rightarrow 0$ lorsque $\nu \geqslant \sigma \rightarrow \infty$ dans un voisinage de $(\mathbf{0}, \mathbf{0})$ il existe un $k_{0}$, un $(\boldsymbol{p}, \boldsymbol{q}) \in \Upsilon_{\mathbf{0}}^{m+n}, \boldsymbol{p} \geqslant \mathbf{1}, \boldsymbol{q} \geqslant \mathbf{1}$, et une suite $\left\{g_{v_{\sigma}}\right\}_{k_{0} \leqslant \sigma \leqslant}$ de fonctions continues dans $\bar{Q}_{00}$ telles que $T\left(\lambda_{v} \boldsymbol{x}, \lambda_{\sigma} \boldsymbol{y}\right)=D_{\boldsymbol{x}}^{\boldsymbol{p}} D_{\boldsymbol{y}}^{\boldsymbol{q}} g_{v \sigma}(\boldsymbol{x}, \boldsymbol{y})$ $=D g_{v \sigma}(\boldsymbol{x}, \boldsymbol{y})$ 'dans $Q_{00}$, pour $\nu \geqslant \sigma \geqslant k_{0}$, et $g_{v \sigma} \rightarrow 0$ uniformément dans $\overline{\boldsymbol{Q}}_{00}$ pour $\nu \geqslant \sigma \rightarrow \infty$. Il en résulte que $\left|g_{v \sigma}(\boldsymbol{x}, \boldsymbol{y})\right| \leqslant \varepsilon_{\sigma}$ dans $\bar{Q}_{00}$ pour $v \geqslant \sigma \geqslant k_{0}$, où

$$
\varepsilon_{\sigma} \rightarrow 0 \quad \text { en décroissant. }
$$

Si l'on pose $G_{v \sigma}(\boldsymbol{x}, \boldsymbol{y})=\lambda_{v}^{|\boldsymbol{p}|} \lambda_{\sigma}^{|\boldsymbol{\alpha}|} g_{v \sigma}\left(\frac{\boldsymbol{x}}{\lambda_{p}}, \frac{\boldsymbol{y}}{\lambda_{\sigma}}\right)$ dans $\bar{Q}_{v \sigma}$, on trouve

$$
D G_{\nu \sigma}=T \quad \text { dans } \quad Q_{v \sigma}
$$

$$
\left|G_{v \sigma}(\boldsymbol{x}, \boldsymbol{y})\right| \leqslant \lambda_{v}^{|\boldsymbol{p}|} \lambda_{\sigma}^{|\boldsymbol{\alpha}|} \varepsilon_{\sigma} \quad \text { dans } \quad \bar{Q}_{\eta \sigma} \text { pour } v \geqslant \sigma \geqslant k_{0}
$$

Fixons maintenant un $\sigma \geqslant k_{0}$. D'après (6.5.4) la fonction $q_{v}=G_{\sigma+v+1, \sigma}-$ $-G_{\sigma+\nu, \sigma}$ est du type $\mathcal{K}_{\boldsymbol{p}, \boldsymbol{q}}$ et on a $\left|q_{\nu}(\boldsymbol{x}, \boldsymbol{y})\right| \leqslant 2 \lambda_{\sigma+\nu}^{|\boldsymbol{p}|} \lambda_{\sigma}^{|\boldsymbol{\alpha}|} \varepsilon_{\sigma}$ dans $\vec{Q}_{\sigma+\nu+1, \sigma} . \mathrm{Il}$ en résulte, d'après le lemme $2 \mathrm{du} \mathrm{N}^{0} 2.4$, que la fonction $q_{v}$ possède un prolongement $\bar{q}_{,}$du type $\chi_{\boldsymbol{p}, \boldsymbol{q}}$ sur $\mathcal{E}^{m+n}$ tel que

$$
\left|\bar{q}_{\nu}(\boldsymbol{x}, \boldsymbol{y})\right| \leqslant 2 K \lambda_{\sigma+v}^{|\boldsymbol{p}|} \lambda_{\sigma}^{|\boldsymbol{q}|} \varepsilon_{\sigma} N_{\boldsymbol{p}, \boldsymbol{q}}\left(\frac{\boldsymbol{x}}{\lambda_{\sigma+v+1}}, \frac{\boldsymbol{y}}{\lambda_{\sigma}}\right)
$$

(où $K$ ne dépend pas de $\boldsymbol{x}, \boldsymbol{y}, \sigma, \nu$ ). En posant $\bar{G}_{n}=G_{\sigma \sigma}+-\bar{q}_{0}+\ldots+\bar{q}_{t-1}$ dans $\bar{Q}_{\sigma}(v=1,2, \ldots)$, on obtient, d'après (6.5.4),

(6.5.6) $\quad \bar{D} G_{\nu}=T$ dans $Q_{\sigma} \quad$ et $\quad\left|\bar{G}_{\nu}(\boldsymbol{x}, \boldsymbol{y})\right| \leqslant \lambda_{\sigma+\nu}^{|\boldsymbol{p}|} \lambda_{\sigma}^{|\boldsymbol{\alpha}|} \varepsilon_{\sigma}$ dans $\bar{Q}_{\sigma+\nu, \sigma}$

car, en vertu de la définition des $\bar{q}_{v}$ et ' $q_{v}, \bar{G}_{v}=G_{\sigma+\nu \sigma}$ dans $\bar{Q}_{\sigma+\nu, \sigma}$. L'inégalité (6.5.5) donne (en tenant compte de (6.5.2) et (2.3.3)) la majoration suivante dans $\bar{Q}_{\sigma}$ :

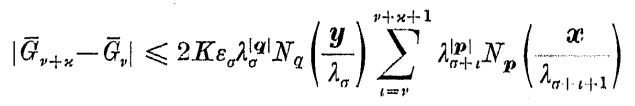

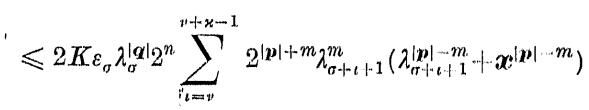

$$
\begin{aligned}
& \leqslant K_{1} \varepsilon_{\sigma} \lambda_{\sigma}^{|\boldsymbol{\alpha}|} \lambda_{\sigma+\nu}^{m}\left(\lambda_{\sigma+\nu}^{|\boldsymbol{p}|-m}+|\boldsymbol{x}|^{|\boldsymbol{p}|-m}\right),
\end{aligned}
$$

où $K_{1}$ ne dépend pas de $\boldsymbol{x}, \boldsymbol{y}, \nu, x, \sigma$. On en conclut que la suite $\left\{\bar{G}_{v}\right\}$ converge uniformément dans $\bar{Q}_{\sigma}$; sa limite $F_{\sigma}$ est done continue et

$$
D F_{\sigma}=T \quad \text { dans } \vec{Q}_{\sigma} \text {. }
$$

En vertu de (6.5.6) on a $\left|\boldsymbol{F}_{\sigma}(\boldsymbol{x}, \boldsymbol{y})\right| \leqslant\left(1+2 \boldsymbol{K}_{1}\right) \varepsilon_{\sigma} \lambda_{\sigma}^{|\boldsymbol{\alpha}|} \lambda_{\sigma+\nu}^{|\boldsymbol{p}|}$ dans $\bar{Q}_{\sigma+r, \sigma}$, ce qui donne facilement

$$
\left|F_{\sigma}(\boldsymbol{x}, \boldsymbol{y})\right| \leqslant K_{2} \varepsilon_{\sigma} \lambda_{\sigma}^{|\boldsymbol{\alpha}|}|\boldsymbol{x}|^{|\boldsymbol{p}|} \quad \text { dans } \bar{Q}_{\sigma},
$$

où $K_{0}$ ne dépend pas de $\boldsymbol{x}, \boldsymbol{y}, \boldsymbol{\sigma}$.

Considérons la suite $\left\{F_{\sigma}\right\}$ et procédons encore une fois comme pour la suite $\left\{G_{\sigma v}\right\}$. D'après $(6.5 .7)$ et (6.5.8) la fonction $r_{\sigma}=F_{\sigma}-F_{\sigma-1}$ $(\sigma=1,2, \ldots)$ est du type $\mathcal{K}_{\boldsymbol{p}, \boldsymbol{q}}$ et on a $\left|r_{\sigma}\right| \leqslant 2 K_{2} \varepsilon_{\sigma-1} \lambda_{\sigma-1}^{|\boldsymbol{q}|}|\boldsymbol{x}|^{|\boldsymbol{p}|}$ dans $\bar{Q}_{\sigma}$, donc, selon le lemme $3 \mathrm{du} \mathrm{N}^{\circ} 2.4$, elle possède un prolongement $\bar{r}_{\sigma} \mathrm{du}$ type $\varkappa_{\boldsymbol{p}, \boldsymbol{q}}$ sur $\varepsilon^{m+n}$ tel que

$$
\left|\bar{r}_{\sigma}\right| \leqslant K_{3} \varepsilon_{\sigma-1} \lambda_{\sigma-1}^{|\boldsymbol{\alpha}|}|\boldsymbol{x}|^{|\boldsymbol{p}|} N_{\boldsymbol{q}}\left(\frac{\boldsymbol{y}}{\lambda_{\sigma}}\right),
$$

où $K_{3}$ ne dépend pas de $\boldsymbol{x}, \boldsymbol{y}, \sigma$. En posant $\overline{\boldsymbol{F}}_{\sigma}=F_{k_{0}}+\bar{r}_{k_{0}+1}+\ldots+\bar{r}_{\sigma}$ dans $Q_{k_{0}}\left(\sigma \geqslant k_{0}\right)$, on obtient, d'après $(6.5 .7)$ et (6.5.8),

(6.5.10) $D \bar{F}_{\sigma}=T$ dans $Q_{k_{0}}$ et $\left|\bar{F}_{\sigma}(\boldsymbol{x}, \boldsymbol{y})\right| \leqslant K_{2} \varepsilon_{\sigma} \lambda_{\sigma}^{|\boldsymbol{\alpha}|}|\boldsymbol{x}|^{|\boldsymbol{p}|}$ dans $Q_{\sigma}$.

L'inégalité (6.5.9) donne (en tenant compte de (6.5.2) et (2.3.3)) la majoration suivante dans $Q_{k_{0}}$ :

$$
\left|\bar{F}_{\sigma+x}-\bar{F}_{\sigma}\right| \leqslant K_{4} \varepsilon_{\sigma}|\boldsymbol{x}|^{|\boldsymbol{p}|} \lambda_{\sigma}^{n}\left(\lambda_{\sigma}^{|\boldsymbol{\alpha}|-n}+|\boldsymbol{x}|^{|\boldsymbol{q}|-n}\right),
$$

où $K_{4}$ ne dépend pas de $\boldsymbol{x}, \boldsymbol{y}, \varkappa, \sigma$. Il en résulte que la suite $\left\{\overline{\boldsymbol{F}}_{\sigma}\right\}$ converge uniformément dans $Q_{k_{0}}$; sa limite $F$ est continue et on a (cf. (6.5.10)) $D F=T$ dans $Q_{k_{0}} ;$ d'après (6.5.10) nous avons de plus $|\boldsymbol{F}(\boldsymbol{x}, \boldsymbol{y})| \leqslant$ $\leqslant\left(K_{2}+2 K_{4}\right) \varepsilon_{\sigma}|\boldsymbol{x}| \boldsymbol{| \boldsymbol { p }} \boldsymbol{\lambda}_{\boldsymbol{\sigma}}^{|\boldsymbol{\alpha}|}$ dans $Q_{\sigma}$, ce qui donne facilement $|\bar{F}(\boldsymbol{x}, \boldsymbol{y})| \leqslant$ $\leqslant K_{5} \varepsilon(\boldsymbol{x}, \boldsymbol{y})|\boldsymbol{x}|^{|\boldsymbol{p}|}(|\boldsymbol{x}|+|\boldsymbol{y}|)^{|\boldsymbol{q}|}$ dans $Q_{k_{0}}$, où $K_{5}$ ne dépend pas de $\boldsymbol{x}, \boldsymbol{y}$ et lim $\varepsilon(\boldsymbol{x}, \boldsymbol{y})=0$. Le théorème est ainsi démontré.

$\lim _{x \rightarrow 0, y \rightarrow 0}$

\section{Valeur, fixation et valeur de section $\left.{ }^{89}\right)$}

7.1. Relation en un point. Supposons qu'on puisse fixer $\boldsymbol{x}=\boldsymbol{x}_{0}$ sur un voisinage de $\boldsymbol{y}_{0}$ dans une distribution $T(\boldsymbol{x}, \boldsymbol{y})$ et soit $S(\boldsymbol{y})=T\left(\boldsymbol{x}_{0}, \boldsymbol{y}\right)$. L'existence de la valeur $T\left(\boldsymbol{x}_{0}, \boldsymbol{y}_{0}\right)$ n'entraîne pas celle de $S\left(\boldsymbol{y}_{0}\right)$, ainsi que celle de $S\left(\boldsymbol{y}_{0}\right)$ n'entraîne pas celle de $T\left(\boldsymbol{x}_{0}, \boldsymbol{y}_{0}\right)$. Voici des exemples (dans le cas $m=n=1$ ). Si $\boldsymbol{T}$ est la fonction caractéristique de l'ensemble $|x| \leqslant y^{2}, y \geqslant 0, S(y)$ est la fonction d'Heaviside; on a alors $T^{\prime}(0,0)=0$, tandis que $S(0)$ n'existe pas. Si $T$ est la fonction caractéristique de l'ensemble $|x| \leqslant|y|, S(y)=1$; on a alors $S(0)=1$, tandis que $T(0,0)$ n'existe pas. Il peut aussi arriver que toutes les deux valeurs existent et soient différentes. En effet, si $T$ est la fonction caractéristique de l'ensemble

$\left.{ }^{29}\right)$ Tous les théorèmes des $\S \S 7$ et 8 ont lieu aussi dans le cas de la limite Studia Mathematica T. XVII 
$|x| \leqslant y^{2}$, on a $S(y)=1$, donc $S(0)=1$, tandis que $T(0,0)=0$. Nous avons pourtant le

THÉOR亡̀me. Si la valeur $T\left(\boldsymbol{x}_{0}, \boldsymbol{y}_{0}\right)$ directionnelle par rapport à $\boldsymbol{y}$ existe, alors la valeur $S\left(\boldsymbol{y}_{0}\right)$ existe et on a $S\left(\boldsymbol{y}_{0}\right)=T\left(\boldsymbol{x}_{0}, \boldsymbol{y}_{0}\right)$.

Démonstration. En effet, l'existence des limites

$$
\lim _{\lambda \rightarrow 0+}\left(T, \frac{1}{\lambda^{m}} \varphi\left(\frac{\boldsymbol{x}-\boldsymbol{x}_{\mathbf{0}}}{\lambda}\right) \frac{1}{\mu^{m}}\left(\frac{\boldsymbol{y}-\boldsymbol{y}_{\mathbf{0}}}{\mu}\right)\right)=\left(S, \frac{1}{\mu^{2}} \psi\left(\frac{\boldsymbol{y}-\boldsymbol{y}_{0}}{\mu}\right)\right)
$$

$$
\lim _{0<\lambda \leqslant \mu \rightarrow 0}\left(T, \frac{1}{\lambda^{m}} \varphi\left(\frac{\boldsymbol{x}-\boldsymbol{x}_{0}}{\lambda}\right) \frac{1}{\mu^{n}} \psi\left(\frac{\boldsymbol{y}-\boldsymbol{y}_{0}}{\mu}\right)\right)=T\left(\boldsymbol{x}_{0}, \boldsymbol{y}_{0}\right)
$$

entraîne celle de la limite itérée

$$
S\left(\boldsymbol{x}_{0}\right)=\lim _{\mu \rightarrow 0+}\left(S, \frac{1}{\mu^{n}} \psi\left(\frac{\boldsymbol{y}-\boldsymbol{y}_{0}}{\mu}\right)\right) .
$$

Remarque. On a un théorème analogue pour la valeur par rapport à un système de directions.

7.2. Relation intégrale pour la valeur. Du fait que la section $S(\boldsymbol{y})=T\left(\boldsymbol{x}_{0}, \boldsymbol{y}\right)$ existe on ne peut tirer aucune conséquence sur l'existence de la valeur de $T$ aux points de l'hyperplan $x=x_{0}$. Par exemple, la distribution $(n=m=1)$

$$
T=\sum_{\nu \mid 1}^{\infty} \frac{1}{2^{v}} \delta\left(x-\frac{1}{2^{v}}\right) \cos 2^{v} y
$$

ne possède de valeur en aucun des points $(0, y)$ bien que $T(0, y)=0$. En effet, pour tout $\psi \epsilon(D)_{y}$ la mesure

$$
U_{y}=(T, \psi(y))_{y}=\sum_{\nu \mid 1}^{\infty} \frac{c_{\nu}}{2^{\nu}} \delta\left(x-\frac{1}{2^{\nu}}\right)
$$

possède la densité 0 en 0 , car $c_{v}=\int \psi(y) \cos 2^{y} y d y \rightarrow 0$ lorsque $y \rightarrow \infty$ il en résulte (selon la proposition $4 \mathrm{du} \mathrm{N}^{\circ} 4.6$ ) que $T(0, y)=0$. Cependant, quel que soit $y_{0}$, la suite $\left\{T\left(x / 2^{y}, y_{0}+y / 2^{y}\right)\right\}$ ne converge vers aucune constante, puisque $T\left(x / 2^{y}, y / 2^{y}\right)=\delta(x-1) \cos \left(2^{y} y_{0}+y\right)$ dans la bande $\frac{1}{2}<x<2$.

En revanche, si l'on suppose qu'une distribution $T(\boldsymbol{x}, \boldsymbol{y})$ possède une valeur en chaque point de l'hyperplan $x=x_{0}$, on peut en tirer une conclusion sur la possibilité de fixer $\boldsymbol{x}=\boldsymbol{x}_{0}$. Nous avons en effet le

THÉokÈME. Supposons que la valeur $T\left(\boldsymbol{x}_{0}, \boldsymbol{y}_{0}\right)$ existe pour tout $\boldsymbol{y}_{0} \in G$, où $G$ est un ouvert de $\left(\mathcal{E}^{n}\right)_{\boldsymbol{y}}$. Il existe un ouvert $G_{1}$ dense dans $G$, tel que dans
$T$ on puisse fixer $\boldsymbol{x}=\boldsymbol{x}_{0}$ sur $G_{1}$, que la section $S(\boldsymbol{y})=T\left(\boldsymbol{x}_{0}, \boldsymbol{y}\right)$ soit une fonction localement sommable et qu'on ait $S\left(\boldsymbol{y}_{0}\right)=T\left(\boldsymbol{x}_{0}, \boldsymbol{y}_{0}\right)$ pour presque tous les $\boldsymbol{y}_{0} \in G_{1}$.

Démonstration. Il suffit de prouver que toute boule contenue dans $G$ contient une boule dans laquelle la conclusion du théorème est vrai. Considérons la fonctionnelle

$$
\begin{aligned}
T_{\lambda}[\boldsymbol{\eta}, \chi(\boldsymbol{x}, \boldsymbol{y})] & =\left(T\left(\boldsymbol{x}_{0}+\lambda \boldsymbol{x}, \boldsymbol{\eta}+\lambda \boldsymbol{y}\right), \chi(\boldsymbol{x}, \boldsymbol{y})\right) \\
& =\left(T, \frac{1}{\lambda^{m+n}} \chi\left(\frac{\boldsymbol{x}-\boldsymbol{x}_{0}}{\lambda}, \frac{\boldsymbol{y}-\boldsymbol{\eta}}{\lambda}\right)\right)
\end{aligned}
$$

et soit $K$ une boule fermée contenue dans $G$. Désignons par $Q$ l'ensemble $\left|\boldsymbol{x}-\boldsymbol{x}_{0}\right| \leqslant 1,|\boldsymbol{y}| \leqslant 1$. II existe alors un entier positif $k$ et un $\lambda_{0}>0$ tels que si $0<\lambda \leqslant \lambda_{0}$, la fonctionnelle $T_{\lambda}$ est continue dans l'espace métrique complet $K \times D_{Q}^{k_{i}}$. D'après les hypothèses on a

$$
\text { (7.2.3) } \lim _{\lambda \rightarrow 0+} T_{\lambda}[\eta, \chi]=T\left(x_{0}, \eta\right) \int \chi d x d y \quad \text { pour } \quad \eta \in K, \chi \in \mathcal{D}_{Q}^{k} \text {, }
$$

d'où il résulte que si l'on fixe $\eta$ et $\chi$, alors $T_{\lambda}(\eta, \chi)$ est borné pour $0<\lambda \leqslant \lambda_{0}$, car elle est continue aussi par rapport à $\lambda$. Selon le théorème de Baire $T_{\lambda}(\eta, \chi)$ est donc uniformément bornée pour $0<\lambda \leqslant \lambda_{0}$ dans une boule de $K \times D_{Q}^{k}$, ou pour $\eta \in K_{0}$ et $\left\|\chi-\chi_{0}\right\|_{k}<\varepsilon_{0}$, où $K_{0}$ est une boule ouverte contenue dans $K, \chi_{0} \epsilon D_{Q}^{k}, \varepsilon_{0}>0$. Puisque $T_{\lambda}$ est linéaire par rapport à $\chi$, on en conclut qu'il existe une constante $M$ telle que

$$
\text { (7.2.4) } \quad\left[T_{\lambda}[\eta, \chi] \mid \leqslant M\|\chi\|_{k} \quad \text { lorsque } \quad 0<\lambda \leqslant \lambda_{0}, \eta \in K_{0}, \chi \in \mathbb{D}_{Q}^{k}\right. \text {. }
$$

Soit $\psi \epsilon \mathcal{D}_{\mathcal{K}_{0}}$ et $\varphi \epsilon D_{\left|x-x_{0}\right| \leqslant 1}$ telle que $\int \varphi d x=1$. Soit $\beta \epsilon\left(\mathcal{D}_{|y| \leqslant 1}\right.$ telle que $\int \beta d y=1$. En vertu de $(7.2 .2)$, on a

$$
\begin{aligned}
(7.2 .5)\left(T, \frac{1}{\lambda^{m}} \varphi\left(\frac{\boldsymbol{x}-\boldsymbol{x}_{0}}{\lambda}\right) \psi(\boldsymbol{y})\right) & =\int_{K_{0}}\left(T, \frac{1}{\lambda^{m}} \varphi\left(\frac{\boldsymbol{x}-\boldsymbol{x}_{0}}{\lambda}\right) \psi(\boldsymbol{y}) \frac{1}{\lambda^{n}} \beta\left(\frac{\boldsymbol{y}-\boldsymbol{\eta}}{\lambda}\right)\right) d \boldsymbol{\eta} \\
& =\int_{K_{0}} T_{\lambda}[\boldsymbol{\eta}, \varphi(\boldsymbol{x}) \psi(\boldsymbol{\eta}+\lambda \boldsymbol{y}) \beta(\boldsymbol{y})] d \boldsymbol{\eta},
\end{aligned}
$$

pourvu que $\lambda$ soit suffisamment petit. Si $\eta \in K_{0}$ et $\lambda \rightarrow 0$, on a, d'après (7.2.3), $T_{\lambda}[\boldsymbol{\eta}, \varphi(\boldsymbol{x}) \psi(\boldsymbol{\eta}) \beta(\boldsymbol{y})] \rightarrow T^{\prime}\left(\boldsymbol{x}_{0}, \boldsymbol{\eta}\right) \psi(\boldsymbol{\eta})$ et, d'après (7.2.4), $T_{\lambda}[\boldsymbol{\eta}, \varphi(\boldsymbol{x})(\psi(\boldsymbol{\eta}+\lambda \boldsymbol{y})-\psi(\boldsymbol{\eta})) \beta(\boldsymbol{y})] \rightarrow 0$, d'où il résulte que l'expression $T_{\lambda}[\boldsymbol{\eta}, \varphi(\boldsymbol{x}) \psi(\boldsymbol{\eta}+\lambda \boldsymbol{y}) \beta(\boldsymbol{y})]$ converge vers $T\left(\boldsymbol{x}_{0}, \eta\right) \psi(\eta)$. De plus, grâce à (7.2.4), cette expression reste uniformément bornée pour $\lambda \rightarrow 0$ et $\eta \in K_{0}$. En intégrant on obtient donc (en tenant compte de (7.2.5))

$$
\left(T, \frac{1}{\lambda^{m}} \varphi\left(\frac{\boldsymbol{x}-\boldsymbol{x}_{0}}{\lambda}\right) \psi(\boldsymbol{y})\right) \rightarrow \int T\left(x_{0}, \boldsymbol{\eta}\right) \psi(\boldsymbol{\eta}) d \boldsymbol{\eta},
$$


ce qui termine la démonstration (d'après le théorème du $\mathrm{N}^{0} 4.5$ ).

Les hypothèses du théorème ne suffisent pas pour l'existence de la section $T\left(\boldsymbol{x}_{0}, \boldsymbol{y}\right)$ sur $G$ tout entier. Considérons, par exemple, la distribution ( $m=n=1$ )

$$
T=\sum_{\nu \mid 1}^{\infty} \frac{1}{5^{p}} \delta\left(x-\frac{1}{6^{\nu}}\right) \delta\left(y-\frac{1}{2^{\nu}}\right) .
$$

On vérifie que $T\left(0, y_{0}\right)=0$ pour tout $y_{0}$, tandis qu'on ne peut pas fixer $x=0$ sur le voisinage de $y=0$. En tenant compte de cet exemple on construit sans peine une mesure qui possède la valeur 0 en chaque point de l'axe $y$, pour laquelle tout ouvert de $y$, sur lequel on peut fixer $x=0$, fait partie d'un ouvert dense donné arbitrairement.

7.3. Relation' intégrale pour la valeur directionnelle. Si l'on suppose dans le théorème du $\mathrm{N}^{0} 7.2$ que les valeurs $T\left(\boldsymbol{x}_{0}, \boldsymbol{y}_{0}\right)$ sont directionnelles par rapport à $\boldsymbol{y}$, on obtient l'existence de la section $T\left(\boldsymbol{x}_{0}, \boldsymbol{y}\right)$ dans $G$ tout entier. En effet, nous avons:

THÉORÈME. Soit $G_{0}$ un ouvert contenu dans un ouvert $G \subset\left(\varepsilon^{(n)}\right)_{y}$. Supposons que dans une distribution $T(\boldsymbol{x}, \boldsymbol{y})$ on puisse fixer $\boldsymbol{x}=\boldsymbol{x}_{0}$ sur $G_{0}$ et que la valeur $T\left(\boldsymbol{x}_{0}, \boldsymbol{y}_{0}\right)$ directionnelle par rapport à y existe pour tout $\boldsymbol{y}_{0} \in G-G_{0}$. Alors on peut fixer $\boldsymbol{x}=\boldsymbol{x}_{0}$ sur $\boldsymbol{G}$ tout entier.

La démonstration sera basée sur le lemme suivant:

LEMve. Soient $\psi, \beta \in\left(D_{y}\right)$ des fonctions $\geqslant 0$ telles que $\int \psi d y=\int \beta d y$ $=1$ et posons

$$
\psi_{\mu}(\boldsymbol{y})=\frac{1}{\mu^{n}} \psi\left(\frac{\boldsymbol{y}}{\mu}\right)
$$

Soit $\left\{\boldsymbol{y}_{v}\right\}$ une suite de points telle que les supports des fonctions $\beta_{v}(\boldsymbol{y})$ $=\beta\left(\boldsymbol{y}-\boldsymbol{y}_{v}\right)$ soient contenus dans un compact $E$, et soit $\left\{S_{v}\right\}$ une suite de distributions, bornée dans un ouvert contenant $E$. Si

$$
\left(S_{v}, \beta_{v}\right)>\varepsilon>\bar{\varepsilon}>0 \quad(v=1,2, \ldots)
$$

alors pour chaque $\mu$ suffisamment petit il existe des $\eta_{p} \epsilon$ support de $\beta_{\|}$tels que $\left(S_{v}, \psi_{\mu}\left(\boldsymbol{y}-\eta_{v}\right)\right)>\bar{\varepsilon}(v=1,2, \ldots)$.

Démonstration du lemme. Il existe un ouvert $G \supset E$, un $\left.\eta_{i}\right\rangle_{i_{0}}$ et une constante $M$ tels que $\left|\left(S_{v}, \chi\right)\right| \leqslant M\left\|_{\chi}\right\|_{k}$ pour $\chi \in \mathscr{D}_{(x}$. On en conclut qu'il existe un $\mu_{0}>0$ tel que pour $\mu<\mu_{0}, v=1,2, \ldots$, on a $\left|\left(S_{\nu}, \beta_{\nu} * \psi_{\mu}-\beta_{v}\right)\right|<\varepsilon-\bar{\varepsilon}$ ce qui donne, d'après (7.3.1),

$\int \beta_{v}(\boldsymbol{\eta})\left(S_{v}, \psi_{\mu}(\boldsymbol{y}-\boldsymbol{\eta})\right)_{\boldsymbol{y}} d \boldsymbol{\eta}=\left(S_{\nu}, \beta_{\nu} * \psi_{\mu}\right)>\left(S_{\nu} ; \beta_{v}\right)-(\varepsilon-\bar{\varepsilon})>\bar{\varepsilon}=\bar{\varepsilon} \int \beta_{\nu}(\boldsymbol{\eta}) d \boldsymbol{\gamma}_{\boldsymbol{\gamma}}$.

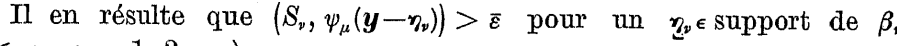
$\left(\mu<\mu_{0}, v=1,2, \ldots\right)$.

Démonstration du théorème. On peut admettre $\boldsymbol{x}_{0}=0$. Supposons, par l'impossible, qu'on ne puisse pas fixer $\boldsymbol{x}=\boldsymbol{x}_{0}$ sur $G$. Selon le corollaire 2 du $\mathrm{N}^{0}$ 6.1, il existe donc une $\varphi \epsilon(D)_{x}$ et une $\bar{\psi} \epsilon \mathcal{D}_{G}$ telles que $(T(\boldsymbol{A} \boldsymbol{x}, \boldsymbol{y}), \varphi(\boldsymbol{x}) \bar{\psi}(\boldsymbol{y}))$ diverge lorsque $|\boldsymbol{A}| \rightarrow 0$ de façon que $|\boldsymbol{A}|^{m}=O(\operatorname{det} \boldsymbol{A}) ;$ on peut même exiger qu'on ait $\bar{\psi} \geqslant 0$ et $\int \bar{\psi} d y=1$. $\Pi$ en résulte qu'il existe un $\varepsilon_{0}>0$ et des suites $\left\{\boldsymbol{A}_{v}\right\},\left\{\overline{\boldsymbol{A}}_{\nu}\right\}$ telles que $\lambda_{\nu}=\max \left(\left|\overline{\boldsymbol{A}}_{v}\right|,\left|\dot{\boldsymbol{A}}_{\boldsymbol{v}}\right|\right) \rightarrow 0$, $\left|\boldsymbol{A}_{v}\right|^{n}=O\left(\operatorname{det} \boldsymbol{A}_{v}\right),\left|\overline{\boldsymbol{A}}_{v}\right|=O\left(\operatorname{det} \overline{\boldsymbol{A}}_{v}\right)$ et

$$
\left(S_{\nu}, \bar{\psi}\right)_{y}>\varepsilon_{0}
$$

où $\boldsymbol{S}_{v}=\left(T\left(\overline{\boldsymbol{A}}_{v} \boldsymbol{x}, \boldsymbol{y}\right)-T\left(\boldsymbol{A}_{v} \boldsymbol{x}, \boldsymbol{y}\right), \varphi(\boldsymbol{x})\right)_{x}$ sont 'définies dans tout ouvert borné $\Omega$ tel que $\bar{\Omega} \subset G$, pourvu que $\nu$ soit suftisamment grand. Comme la section $T(\mathbf{0}, \boldsymbol{y})$ existe sur $G_{0}$, on a, d'après le théorème $1 \mathrm{du} \mathrm{N}^{0} 6.1$, $S_{v} \rightarrow 0$ dans $\Omega \cap G_{0}$, et par suite

$$
\left(S_{\nu}, \chi\left(\boldsymbol{y}-\xi_{v}\right)\right)_{\boldsymbol{y}} \rightarrow 0, \quad \text { si } \quad \chi \in \mathscr{D}_{G_{0}} \quad \text { et } \quad \xi_{v} \rightarrow 0 .
$$

Soit $\eta \in G-G_{0}$; comme la valeur $T\left(x_{0}, \eta\right)$ directionnelle par rapport à $y$ existe, on a, d'après la proposition 2 du N $N^{0} 6.4, S_{\nu}(\eta+\sigma y) \rightarrow 0$ pourvu que $\lambda_{\nu} \leqslant \sigma \rightarrow 0$. Il existe done un $l=l(\eta)$ tel que

$$
\left|\left(\boldsymbol{S}_{\nu}(\boldsymbol{y}), \chi(\boldsymbol{y})\right)_{\boldsymbol{y}}\right| \leqslant M \varepsilon(\sigma)
$$

lorsque $\lambda_{\nu} \leqslant \sigma, \chi \in \mathcal{D}_{|y-\eta| \leqslant \sigma},\|\chi\|_{l} \leqslant M / \sigma^{n+l}$, où $\lim \varepsilon(\sigma)=0$. Il résulte de (7.3.3) et (7.3.4) que la suite $\left\{S_{\nu}\right\}$ est bornée dans un voisinage de chaque point de $G$; donc elle est bornée dans $G$.

Soit $\psi$ une fonction $\geqslant 0$ de $\mathcal{D}_{|\boldsymbol{y}| \leqslant 1}$, telle que $\int \psi d \boldsymbol{y}=\mathbf{1}$ et soit

$$
\varepsilon_{0}>\varepsilon_{1}>\varepsilon_{2}>\ldots>\frac{1}{2} \varepsilon_{0}>0 .
$$

Désignons par $E_{\mu}$ l'ensemble des $\boldsymbol{y}$ tels que $\varrho(\boldsymbol{y}$, support de $\bar{\psi}) \leqslant \mu$ et soit $\mu_{0}>0$ tel que

$$
E_{\mu_{0}} \subset G \text {. }
$$

Nous définirons par récurrence sur $k$ les $\mu_{k}$ et $\left\{\eta_{k_{v}}\right\}$ tels que (7.3.7) $\left(\$_{\nu}, \psi_{\mu_{k}}\left(\boldsymbol{y}-\eta_{k_{\nu}}\right)\right)>\varepsilon_{k}$, support de $\psi_{\mu_{k}}\left(\boldsymbol{y}-\eta_{k_{\nu}}\right) \subset E_{\mu_{1}+\ldots+\mu_{k}}$,

$$
\mu_{k}<\frac{1}{2} \mu_{k-1} \quad(k=1,2, \ldots),
$$

(7.3.9) $\boldsymbol{\eta}_{k v} \in$ support de $\psi_{\mu_{k-1}}\left(\boldsymbol{y}-\boldsymbol{\eta}_{k-1, \nu}\right) \quad(k=2,3, \ldots)$.

D'après le lemme, il résulte de (7.3.2) qu'il existe un $\mu_{1}<\frac{1}{2} \mu_{0}$ et une suite $\left\{\eta_{1 \nu}\right\}$ tels que $\left(S_{\nu}, \psi_{\mu_{1}}\left(\boldsymbol{y}-\boldsymbol{\eta}_{1_{\nu}}\right)\right)>\varepsilon_{1}$ et $\boldsymbol{\eta}_{1_{\nu}} \in$ support de $\bar{\psi}$; alors le support de $\psi_{\mu_{1}}\left(\boldsymbol{y}-\eta_{1_{v}}\right)$ fait partie de $E_{\mu_{1}}$. Ayant défini $\mu_{1},\left\{\eta_{1_{v}}\right\}, \ldots, \mu_{k}$, 
$\left\{\eta_{\text {. }}\right\}$ satisfaisant aux conditions (7.3.7)-(7.3.9), on déduit de $(7.3 .7)^{30}$ ) et (7.3.5), selon le lemme, qu'il existe un $\mu_{k+1}<\frac{1}{2} \mu_{k}$ et une suite $\left\{\eta_{k+1, p}\right\}$ tels que $\left(S_{v}, \psi_{\mu_{k+1}}\left(\boldsymbol{y}-\eta_{k+1, \nu}\right)\right)>\varepsilon_{k+1}$ et $\boldsymbol{\eta}_{k+1} \epsilon$ support de $\psi_{\mu_{k}}\left(\boldsymbol{y}-\boldsymbol{\eta}_{k_{v}}\right)$; alors d'après (7.3.7), le support de $\psi_{\mu_{k+1}}\left(\boldsymbol{y}-\boldsymbol{\eta}_{k+1, v}\right)$ fait partie de $\mathbb{E}_{\mu_{1}+\ldots+\mu_{k}+\mu_{k+1}}$.

En vertu de (7.3.8) on a $\mu_{1}+\ldots+\mu_{k}<\mu_{0}$, donc, d'après (7.3.7) et (7.3.9),

$$
\eta_{k v} \in E_{\mu_{0}} \quad(k, v=1,2, \ldots) .
$$

D'après le „procedé diagonal" bien connu il existe une suite d'indices $\left\{\alpha_{v}\right\}$ telle que

(7.3.11) $\quad \eta_{k \alpha_{\nu}} \rightarrow \eta_{k} \quad$ lorsque $\quad v \rightarrow \infty \quad(k=1,2, \ldots)$;

on peut même exiger qu'on ait

$$
\left|\eta_{k a_{k}}-\eta_{k}\right|<\mu_{k}
$$

et

$$
\lambda_{\alpha_{k}}<4 \mu_{k} .
$$

Grâce à (7.3.9) on a $\left|\boldsymbol{\eta}_{k+1, v}-\eta_{k, \nu}\right|=\mu_{k}$, d'où $\left|\eta_{k+1}-\eta_{k}\right| \leqslant \mu_{k}$. Il en résulte, en tenant compte de (7.3.8), que $\eta_{k}$ converge vers un $\eta$ et qu'on a

$$
\left|\eta_{\eta_{0}}-\eta\right| \leqslant 2 \mu_{k} \text {. }
$$

Montrons que $\eta \in G-G_{0}$. D'après (7.3.10) et (7.3.6), $\eta_{\epsilon} G$. On a $\varrho\left(\eta_{k},-G_{0}\right) \leqslant \mu_{k}$, sinon on aurait $\psi_{\mu_{k}}\left(\boldsymbol{y}-\eta_{k}\right) \in \mathcal{D}_{G_{0}}$ et par suite, d'après (7.3.11) et (7.3.3), $\left(S_{a_{\nu}}, \psi_{\mu_{k}}\left(\boldsymbol{y}-\eta_{k \alpha_{\nu}}\right)\right) \rightarrow 0$ pour $\nu \rightarrow \infty$, contrairement à (7.3.7). Il en résulte que $\eta \in-G_{0}$.

On tire de (7.3.7) et (7.3.5)

$$
\left(S_{a_{k}}, \psi_{\mu_{k}}\left(\boldsymbol{y}-\eta_{k a_{k}}\right)\right)>\frac{1}{2} \varepsilon_{0}>0 \quad(k=1,2, \ldots) .
$$

Cependant, d'après (7.3.12) et (7.3.14), on a $\left|\eta_{k c_{k}}-\eta\right|<3 \mu_{k}$, comme $\eta \in G-G_{0}$, il en résulte, selon (7.3.4) et en tenant compte de (7.3.13), que $\mid\left(S_{a_{k}}, \psi_{\mu_{k}}\left(\boldsymbol{y}-\eta_{\left.k a_{k}\right)}\right) \mid \leqslant M \varepsilon\left(4 \mu_{k}\right) \rightarrow 0\right.$ où $M=4^{n+l}\|\psi\|_{l}$, contrairement à (7.3.15). Le théorème est ainsi démontré.

Les théorèmes des $\mathbb{N}^{0} 7.1$ et 7.3 donnent

CoROLLACRE. Si la valeur $T\left(x_{0}, y_{0}\right)$ direetionnelle existe par rapport $\grave{a} y$ pour tout $y_{0} d^{\prime}$ un ouvert $G \subset\left(\mathcal{E}^{n}\right)_{\boldsymbol{y}}$, alors la section $S(\boldsymbol{y})=T\left(\boldsymbol{x}_{0}, \boldsymbol{y}\right)$ existe sur $G$ et on $\boldsymbol{a} S\left(\boldsymbol{y}_{0}\right)=T\left(\boldsymbol{x}_{0}, \boldsymbol{y}_{0}\right)$ pour tout $\boldsymbol{y}_{0} \in G$.

7.4. Cas du produit tensoriel. Faisons encore quelques remarques sur le produit $T(\boldsymbol{x}) S(\boldsymbol{y})$. Le théorème du No 4.5 montre que l'existence

30) D'après (7.3.8) et (7.3.6) on a $E_{\mu_{1}+\ldots+\mu_{k}} \subset E_{\mu_{0}} \subset G$. de la valeur $T\left(\boldsymbol{x}_{0}\right)$ est une condition nécessaire et suffisante pour qu'on puisse fixer $\boldsymbol{x}=\boldsymbol{x}_{0}$ dans $T(\boldsymbol{x}) S(\boldsymbol{y})$; alors $T\left(\boldsymbol{x}_{0}\right) S(\boldsymbol{y})$ est la section. $\mathrm{Si}$ les valeurs $T\left(\boldsymbol{x}_{0}\right)$ et $S\left(\boldsymbol{y}_{0}\right)$ existent, alors $T(\boldsymbol{x}) S(\boldsymbol{y})$ possède en $\left(\boldsymbol{x}_{0}, \boldsymbol{y}_{0}\right)$ une valeur directionnelle par rapport à $\boldsymbol{x}$ et par rapport à $\boldsymbol{y}$ et on a $\left(T \times S^{\prime}\right)\left(\boldsymbol{x}_{0}, \boldsymbol{y}_{0}\right)=T\left(\boldsymbol{x}_{0}\right) S\left(\boldsymbol{y}_{0}\right)$. Si $(T \times S)\left(\boldsymbol{x}_{0}, \boldsymbol{y}_{0}\right)$ et $T\left(\boldsymbol{x}_{0}\right) \neq 0$ existent, alors $\boldsymbol{S}\left(\boldsymbol{y}_{0}\right)$ existe. Par contre, l'existence de la valeur $(T \times S)\left(\boldsymbol{x}_{0}, \boldsymbol{y}_{0}\right)$ n'entraîne pas celle de $T\left(\boldsymbol{x}_{0}\right)$ et $S\left(\boldsymbol{y}_{0}\right)$. On le vérifie à l'exemple suivant $(m=n=1)$ :

$$
T(x)=S(x)=e^{-\sin x \sqrt{1-\ln |x|}}
$$

Pourtant on a la

Proposition. Si le produit $T(\boldsymbol{x}) \boldsymbol{S}(\boldsymbol{y})$ possède en $\left(\boldsymbol{x}_{0}, \boldsymbol{y}_{0}\right)$ une valeur $\neq 0$, directionnelle par rapport à $\boldsymbol{y}$, alors les valeurs $T\left(\boldsymbol{x}_{0}\right)$ et $S\left(\boldsymbol{y}_{0}\right)$ existent.

Démonstration. Admettons $\boldsymbol{x}_{0}=0, \boldsymbol{y}_{0}=0$. Soient $\varphi \in(\mathcal{D})_{\boldsymbol{x}}, \psi \epsilon(\mathcal{D})_{y}$ telles que $\int \varphi d \boldsymbol{x}=\int \psi d \boldsymbol{y}=1$. Si $a_{\lambda}=(T(\lambda \boldsymbol{x}), \varphi(\boldsymbol{x})), \beta_{\mu}=(S(\mu \boldsymbol{y}), \psi(\boldsymbol{y}))$, on trouve

(7.4.2) $\quad a_{\lambda} \beta_{\mu} \rightarrow \gamma=(T \times S)(0,0) \quad$ lorsque $\quad 0<\lambda \leqslant \mu \rightarrow 0$.

Soient $\left\{\lambda_{y}\right\},\left\{\bar{\lambda}_{v}\right\}$ deux suites positives, convergentes vers 0 , et posons $\mu_{v}=\max \left(\lambda_{v}, \bar{\lambda}_{v}\right)$. On tire de (7.4.2) $\alpha_{\bar{\lambda} v} / \alpha_{2, v} \rightarrow 1$ donc $\alpha_{\lambda}$.converge vers une limite $\alpha \neq 0$, pour $\lambda \rightarrow 0$. Il en résulte que la limite $\lim \beta_{\mu}=\gamma / \alpha$ existe et ne dépend pas de $\psi$ ce qui montre que la valeur $\underset{S \rightarrow 0}{S(0)}$ existe.

\section{Ordre de la fixation}

Nous désignerons par $P_{\lambda}\left(\boldsymbol{x}_{0}\right)$ le cube $\left|x_{v}-x_{0}\right|<\lambda(v=1, \ldots, m)$.

Soit $\mathfrak{P}$ un ensemble fini d'élements $\boldsymbol{p}_{1}, \ldots, \boldsymbol{p}_{k} \epsilon \chi_{0}^{m}$; nous désignerons par $\boldsymbol{C}(\mathfrak{P})$ l'ensemble des $\boldsymbol{x} \in \mathcal{E}^{m}$ tels que $0 \leqslant \boldsymbol{x}<\sum_{j \mid 1}^{k} \lambda_{j} \boldsymbol{p}_{j}$ (le signe d'inégalité,$<"<$ se rapporte ici à toutes les coordonnées) pour une suite (dépendant de $x$ ) de nombres $\lambda_{1}, \ldots, \lambda_{k} \geqslant 0$ telle que $\sum_{j \mid 1}^{k} \lambda_{j}=1$.

8.1. Une relation entre les bornes des dérivées. On sait que $\int[\alpha(t)]^{2} d t \leqslant \int\left[\alpha^{\prime}(t)\right]^{2} d t$ pour $\alpha \in \mathcal{D}_{[-1,1]}$. On en tire aisément

(8.1.1) $\quad \int\left|D^{\bar{p}} \varphi\right|^{2} d \boldsymbol{x} \leqslant \int\left|D^{\boldsymbol{p}} \varphi\right|^{2} d \boldsymbol{x} \quad$ lorsque $\quad \varphi \in \mathcal{D}_{[-1,1]}^{m}$ et $\overline{\boldsymbol{p}} \leqslant \boldsymbol{p}$.

Soit $\varphi \subset \mathcal{D}_{[-1,1]} m$ et soit

$$
\varphi(\boldsymbol{x})=\sum_{\boldsymbol{s} \in \mathscr{E}^{m}} a_{\boldsymbol{s}} e^{i \boldsymbol{s x}} \quad\left(\text { dans } \quad(-\pi, \pi)^{m}\right)
$$


le développement de Fourier de $\varphi$. On a $D^{\boldsymbol{p}} \varphi(\boldsymbol{x})=\sum a_{\boldsymbol{s}}(i \boldsymbol{s})^{\boldsymbol{p}} e^{i \boldsymbol{s} \boldsymbol{x}}$. (en admettant $0^{\circ}=1$ ), d'où

$$
\int\left|D^{p} \varphi\right|^{2} d x=\sum\left|a_{s}\right|^{2} s^{2} \boldsymbol{p}
$$

Pour tout $s=\left(s_{1}, \ldots, s_{m}\right) \epsilon \mathscr{E}^{m}$ posons $\hat{\boldsymbol{s}}=\left(\hat{s}_{1}, \ldots, \hat{s}_{m}\right)$, où $\hat{s}_{i}=$ $=\max \left(1,\left|s_{i}\right|\right)$. On a alors $\left|\boldsymbol{s}^{\boldsymbol{p}}\right| \leqslant \hat{\boldsymbol{s}}^{\boldsymbol{p}} \leqslant \hat{\boldsymbol{s}}^{\overrightarrow{\boldsymbol{p}}}$ lorsque $\boldsymbol{p} \leqslant \overline{\boldsymbol{p}}$.

Considérons la série $\sum_{s^{\prime}}\left|a_{\boldsymbol{s}}\right|^{2} \hat{\boldsymbol{s}}^{2 \boldsymbol{p}}$. On a $\hat{\boldsymbol{s}}^{2 \boldsymbol{p}}=\boldsymbol{s}^{2 \boldsymbol{p}^{\prime}}$, où $\boldsymbol{p}^{\prime}=\left(p_{1}^{\prime}, \ldots, p_{m}^{\prime}\right)$

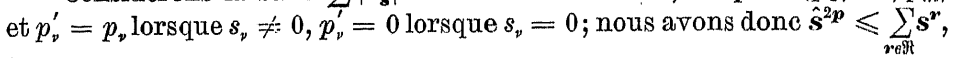
où $\mathscr{R}$ est l'ensemble des $r$ tels que $r_{\nu}=0$ ou $r_{v}=p_{v}$. D'après (8.1.1) et (8.1.3) il en résulte que

$$
\sum_{\boldsymbol{s}}\left|a_{\boldsymbol{s}}\right|^{2} \hat{\boldsymbol{s}}^{2 \boldsymbol{p}} \leqslant 2^{m} \int\left|D^{\boldsymbol{p}} \varphi\right|^{2} d x .
$$

Propostmion $1^{31}$ ). Soit $\mathfrak{P}$ un ensemble fini de $\chi_{0}^{m}$ et $h>0$. Il existe une constante $K=K(\mathfrak{P}, h)$ telle que si $\varphi \in \mathcal{D}_{P_{h}\left(x_{0}\right)}$ et $\int\left|D^{p} \varphi\right|^{2} d x \leqslant M^{2}$ pour $\boldsymbol{p} \in \mathcal{P}$, alors

$$
\int\left|D^{p} \varphi\right|^{2} d x \leqslant K M^{2} \quad \text { pour } \quad \boldsymbol{p} \in O(P)
$$

$$
\left|D^{p} \varphi\right| \leqslant K M \quad \text { lorsque } \quad \boldsymbol{p}+\frac{1}{2} \mathbf{1} \epsilon O(\mathfrak{P}) .
$$

Démonstration. On peut admettre $x_{0}=0, h=1$. Soit $\varphi \in\left(D_{[-1,1]}{ }^{m}\right.$ et supposons que $\int\left|D^{p} \varphi\right| d x \leqslant M^{2}$ pour $\boldsymbol{p} \in \mathfrak{P}$. Considérons le développement (8.1.2). Soient $\boldsymbol{p}_{j} \in \mathcal{P}, \lambda_{j} \geqslant 0, \sum \lambda_{j}=1$. Si $\boldsymbol{p} \geqslant \sum \lambda_{j} \boldsymbol{p}_{j}$, on a $\dot{\boldsymbol{s}}^{2 \boldsymbol{p}} \leqslant \hat{\boldsymbol{s}}^{2 \Sigma_{j} \boldsymbol{p}_{j}}$ $\leqslant \sum \lambda_{j} \hat{\mathbf{s}}^{2_{j}}$ car la fonction $\hat{\boldsymbol{s}}^{z}=\hat{s}_{1}^{z_{1}} \ldots \hat{s}_{m}^{z_{m}}$ est croissante et convexe par rapport à $z$; il en résulte, d'après (8.1.3) et (8.1.4), que

$$
\int\left|D^{\boldsymbol{p}} \varphi\right|^{2} d \boldsymbol{x} \leqslant \sum_{j} \lambda_{j} \sum_{\boldsymbol{s}}\left|\boldsymbol{a}_{\boldsymbol{s}}\right|^{2} \hat{\boldsymbol{s}}^{2 \boldsymbol{p}_{j}} \leqslant 2^{m} \sum_{j} \lambda_{j} \int\left|D^{\boldsymbol{p}_{j}} \varphi\right|^{2} d x \leqslant 2^{m} M .
$$

Si majntenant $\boldsymbol{p}+\frac{1}{2} \mathbf{1}<\sum \lambda_{j} \boldsymbol{p}_{j}$, alors

$$
\boldsymbol{p}+\frac{1+\varepsilon}{2} \mathbf{1}<\sum \lambda_{j} \boldsymbol{p}^{j} \quad \text { (pour un } \varepsilon>0 \text { suffisamment petit), }
$$

done

$$
\left|\mathbf{s}^{\boldsymbol{p}}\right| \leqslant \hat{\boldsymbol{s}}^{\Sigma \lambda_{j} \boldsymbol{p}_{j}-(1+\varepsilon) 1 / 2} \leqslant \sum \lambda_{j} \hat{\boldsymbol{s}}^{\boldsymbol{p}_{j}-(1+\varepsilon) 1 / 2}
$$

31) C'est un théorème du même genre que ceux de Soboleff et de Nikolsky (ef. p. ex. [5] où l'on peut trouver la bibliographie).
En utilisant l'inégalité de Schwartz nous obtenons, d'après (8.1.4),

$$
\begin{aligned}
& \left|D^{\boldsymbol{p}} \varphi\right| \leqslant \sum_{\boldsymbol{s}}\left|a_{\boldsymbol{s}}\right|\left|\boldsymbol{s}^{\boldsymbol{p}}\right| \leqslant \sum_{j} \lambda_{j} \sum_{\boldsymbol{s}}\left|a_{\boldsymbol{s}}\right| \hat{\mathbf{s}}^{\boldsymbol{p}_{j}-(1+\varepsilon) \mathbf{1} / 2} \\
& \leqslant \sum_{j} \lambda_{j} \sqrt{\sum_{\boldsymbol{s}}\left|a_{\boldsymbol{s}}\right|^{2} \hat{\boldsymbol{s}}^{2 \boldsymbol{p}_{j}} \sum \hat{\boldsymbol{s}}^{-(1+\varepsilon) 1}} \leqslant 2^{m / 2} M\left(1+\sum_{1}^{\infty} \frac{2}{v^{1+\varepsilon}}\right)^{m / 2} ;
\end{aligned}
$$

CoRollatRe 1. Si une distribution est d'ordre C $\mathfrak{P}$ dans un ensemble $E$, elle est aussi d'ordre $\subset \mathfrak{P}_{0}$ dans $E$, pourvu que $\mathfrak{P}+\frac{1}{2} 1 \subset C\left(\mathfrak{P}_{0}\right)$.

Proposition 2. Soit $G$ un ouvert de $\left(\mathcal{E}^{m}\right)_{x}$ et soit $E$ un compact, $E \subset G$; sovent $\mathfrak{P}, \mathfrak{P}_{0}$ des ensembles finis de $7_{0}^{m}$ tels que $\mathfrak{P}+\frac{1}{2} \mathbf{1} \subset C\left(\mathfrak{P}_{0}\right)$. Il existe une constante $K=K\left(G, E, \mathfrak{P}, \mathfrak{P}_{0}\right)$ telle que si $T$ est une distribution définie dans $G$ et si

$$
\left|\left(T, \varphi_{1}\left(x_{1}\right) \ldots \varphi_{m}\left(x_{m}\right)\right)\right| \leqslant M, \text { lorsque } \varphi_{1} \ldots \varphi_{m} \in \mathcal{D}_{G} \text { et }\left|D^{p} \varphi_{1} \ldots \varphi_{m}\right| \leqslant
$$

$$
\leqslant 1 \text { pour } \boldsymbol{p} \in \mathcal{P} \text {, }
$$

alors

(8.1.8) $\quad(T, \varphi) \mid \leqslant K M, \quad$ lorsque $\quad \varphi \in \mathcal{D}_{E}$ et $\left|D^{P} \varphi\right| \leqslant 1$ pour $\boldsymbol{p} \epsilon \mathfrak{P}_{0}$.

La condition (8.1.7) entraîne que $T$ est d'ordre $\subset \mathfrak{P}_{0}$ localement dans $G$.

Démonstration. Grâce au lemme sur ,la partition de l'unité" (cf. [7], tome I, p. 23]) le cas général se ramène au cas où $G=(-2,2)^{m}$, $E=[-1,1]^{m}$. Supposons que l'on ait (8.1.7). Fixons une fonction $\gamma \in \mathcal{D}_{(-2,2)}$ telle que $\gamma(t)=1$ dans $[-1,1]$ et posons $a(x)=\gamma\left(x_{1}\right) \ldots \gamma\left(x_{m}\right)$. II existe une constante $K_{0}=K_{0}(\mathfrak{P})$ telle que

(8.1.9) $\quad\left|D^{\boldsymbol{p}}\left(\alpha e^{i \boldsymbol{s} \boldsymbol{x}}\right)\right| \leqslant K_{0}\left|\hat{\boldsymbol{s}}^{\boldsymbol{p}}\right| \quad$ pour $\quad \boldsymbol{s} \in \mathscr{E}^{m}$ et $\boldsymbol{s} \in \mathfrak{P}$.

Si $\overline{\boldsymbol{p}} \in \mathfrak{P}$, on a

$$
\overline{\boldsymbol{p}}+\frac{1+\varepsilon}{2} \mathbf{1}<\sum \lambda_{j} \boldsymbol{p}_{j}, \quad \text { où } \quad \boldsymbol{p}_{j} \in \mathfrak{P}_{0}, \lambda_{j} \geqslant 0, \sum \lambda_{j}=1 \text { et } \varepsilon>\mathbf{0}
$$

par conséquent

$$
\left|\hat{\boldsymbol{s}}^{\overline{\boldsymbol{p}}}\right| \leqslant \hat{\boldsymbol{s}}^{\Sigma \lambda_{j} \boldsymbol{p}_{j}-(1+\varepsilon) 1 / 2} \leqslant \sum \lambda_{j} \hat{\boldsymbol{s}}^{\boldsymbol{p}_{j}-(1+\varepsilon) \mathbf{1} / 2} \leqslant \sum_{\boldsymbol{p} \in \Re_{0}} \hat{\boldsymbol{s}}^{\boldsymbol{p}-(1+\varepsilon) 1 / 2} .
$$

D'après (8.1.7) et (8.1.9) il en résulte que

(8.1.10). $\left|\left(T, \alpha e^{i \boldsymbol{s} x}\right)\right| \leqslant K_{0} M \sum_{\boldsymbol{p} \in \Re_{0}} \hat{\boldsymbol{s}}^{\boldsymbol{p}-(1+\varepsilon) 1 / 2} \quad$ lorsque $\quad \boldsymbol{s} \in \mathscr{E}^{m}$. 
Soit $\varphi \in \mathcal{D}_{[-1,1]} m$ et supposons que $\left|D^{\boldsymbol{p}} \varphi\right| \leqslant 1$ pour $\boldsymbol{p} \in \mathfrak{P}_{0}$. Considérons le développement (8.1.2). On a d'après (8.1.4)

$$
\text { (8.1.11) } \quad \sum\left|a_{\boldsymbol{s}}\right|^{2} \hat{\boldsymbol{s}}^{2 \boldsymbol{p}} \leqslant 4^{m} \quad \text { pour } \quad \boldsymbol{p} \in \mathfrak{P}_{0} \text {. }
$$

Comme $\alpha(\boldsymbol{x})=1$ dans $[-1,1]^{m}$, on a $\varphi=\sum_{\boldsymbol{s}} a_{\boldsymbol{s}} \alpha(\boldsymbol{x}) e^{i \boldsymbol{s} \boldsymbol{x}}$, la série convergeant dans $\mathcal{D}_{(-2,2)^{m}}$, donc $(T, \varphi)=\sum_{\boldsymbol{s}} a_{\boldsymbol{s}}\left(T, \alpha(\boldsymbol{x}) e^{i \boldsymbol{s} \boldsymbol{x}}\right)$. D'après (8.1.10), (8.1.11) et (8.1.4), on obtient donc, en utilisant l'inégalité de Schwartz

$$
\begin{aligned}
|(T, \varphi)| \leqslant K_{0} M \sum_{\boldsymbol{p} \in \Re_{0}} \sum_{\boldsymbol{s}}\left|a_{\boldsymbol{s}}\right| \hat{\boldsymbol{s}}^{\boldsymbol{p}-(1+\varepsilon) 1 / 2} & \leqslant K_{0} M \sum_{\boldsymbol{p} \in \Re_{0}} \sqrt{\sum_{\boldsymbol{s}}\left|a_{\boldsymbol{s}}\right|^{2} \hat{\boldsymbol{s}}^{2 \boldsymbol{p}} \sum_{\boldsymbol{s}} \boldsymbol{s}^{-(1+\varepsilon) 1}} \\
& \leqslant K_{0} M k_{0} 2^{m}\left(1+\sum_{1}^{\infty} \frac{2}{v^{1+s}}\right)^{m / 2}
\end{aligned}
$$

où $k_{0}$ est le nombre d'éléments de $\mathfrak{P}_{0}$. Ainsi nous avons établi (8.1.8).

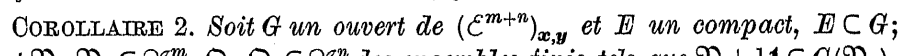
soient $\mathfrak{P}, \mathfrak{P}_{0} \subset \Upsilon_{0}^{m}, \mathfrak{Q}, \mathfrak{Q}_{0} \subset \Upsilon_{0}^{n}$ des ensembles finis tels que $\mathfrak{P}+\frac{1}{2} \mathbf{1} \subset C\left(\mathfrak{P}_{0}\right)$, $\mathfrak{Q}+\frac{1}{2} \mathbf{1} \subset C\left(\mathfrak{Q}_{0}\right)$. Il existe une constante $K=K\left(G, E, \mathfrak{P}, \mathfrak{P}_{0}, \mathfrak{Q}, \mathfrak{Q}_{0}\right)$ telle que si $T$ est une distribution définie dans $G$ et si

(8.1.12) $|(T, \varphi(\boldsymbol{x}) \psi(\boldsymbol{y}))| \leqslant M$, lorsque $\varphi \psi \epsilon \mathscr{D}_{G},\left|D^{\boldsymbol{p}} \varphi\right| \leqslant 1$ pour $p \in \mathfrak{P}$ et

$$
\left|D^{\boldsymbol{\alpha}} \psi\right| \leqslant 1 \text { pour } \boldsymbol{q} \in \mathfrak{Q} \text {, }
$$

alors

(8.1.13) $\quad|(T, \chi(\boldsymbol{x}, \boldsymbol{y}))| \leqslant K M$, lorsque $\chi \in \mathcal{D}_{E}$ et $\left|D_{\boldsymbol{x}}^{\boldsymbol{p}} D_{\boldsymbol{y}}^{q} \chi\right| \leqslant 1$ pour

$$
\boldsymbol{p} \in \mathfrak{P}_{0}, \boldsymbol{q} \in \mathfrak{Q}_{0} !
$$
dans $G$.

La condition (8.1.12) entraîne que $T$ est d'ordre $\subset \mathfrak{P}_{0} \times \mathfrak{Q}_{0}$ localement

Démonstration. On montre que $\left.C\left(\mathfrak{P}_{0}\right) \times C\left(\mathfrak{Q}_{0}\right)=C\left(\mathfrak{P}_{0} \times \mathfrak{Q}_{0}\right)^{32}\right)$. Nous avons done $(\mathfrak{P} \times \mathfrak{Q})+\frac{1}{2} \mathbf{1} C C\left(\mathfrak{P}_{0} \times \mathfrak{Q}_{0}\right)$. Il suffit done de prouver que la condition (8.1.12) entraine la condition (8.1.7) (dans laquelle on a remplacé $\boldsymbol{x}$ par $(\boldsymbol{x}, \boldsymbol{y})$ et $\mathfrak{P}$ par $\mathfrak{P} \times \mathfrak{Q})$. Supposons que $T$ satisfasse à (8.1.1.2) et soit $\varphi_{1}\left(x_{1}\right) \ldots \varphi_{m}\left(x_{m}\right) \psi_{1}\left(y_{1}\right) \ldots \psi_{n}\left(y_{n}\right) \in \mathscr{D}_{Q},\left|D_{\boldsymbol{x}}^{\boldsymbol{p}} D_{\boldsymbol{y}}^{\boldsymbol{\alpha}} \varphi_{1} \ldots \psi_{n}\right| \leqslant 1$ pour $\boldsymbol{p} \in \mathfrak{P}$, $\boldsymbol{q} \in \mathfrak{Q}$. En posant $\gamma_{\boldsymbol{p}}=\sup \left|D_{\boldsymbol{x}}^{\boldsymbol{p}} \varphi_{1} \ldots \varphi_{m}\right|, \delta_{\boldsymbol{\alpha}}=\sup \left|D_{\boldsymbol{y}}^{\boldsymbol{q}} \psi_{1} \ldots \psi_{n}\right|$, on trouve $\gamma_{\boldsymbol{p}} \delta_{\boldsymbol{q}} \leqslant 1$ pour $\boldsymbol{p} \in \mathfrak{P}, \boldsymbol{q} \in \mathfrak{O}$, donc, d'après (8.1.12),

$$
\left|\left(T, \varphi_{1} \ldots \psi_{n}\right)\right| \leqslant M\left(\max _{\mathfrak{B}} \gamma_{p}\right)\left(\max _{\mathfrak{Q}} \delta_{\alpha}\right) \leqslant M, \quad \text { c. q. f. } d .
$$

32) La démonstration est semblable à celle de la propriété analogue de l'enve. loppe convexe.
8.2. Théorèmes sur l'ordre. Soit $\nsupseteq$ un ensemble fini de $\Upsilon_{0}^{m}$. Nous dirons que la valeur $T\left(\boldsymbol{x}_{0}\right)$ est d'ordre $C \mathfrak{P}$ si $\left(T\left(\boldsymbol{x}_{0}+\lambda \boldsymbol{x}\right), \varphi(\boldsymbol{x})\right)$ converge uniformément dans l'ensemble $\left.\varphi \in \mathcal{D}_{(-1,1)}{ }^{m^{33}}\right), \quad\left|D^{\boldsymbol{p}} \varphi\right| \leqslant 1$ pour $\boldsymbol{p} \epsilon \mathfrak{P}$.

Soit $\subseteq$ un ensemble fini de $\Upsilon_{0}^{m+n}$ et supposons qu'on puisse fixer $x=x_{0}$ sur un ouvert $\Omega \subset\left(\mathcal{E}^{n}\right)_{\boldsymbol{y}}$ dans une distribution $T(\boldsymbol{x}, \boldsymbol{y})$. Soit $E \subset \Omega$. Nous dirons que la fixation est d'ordre $\subset \mathcal{S}$ sur $\Omega$, si la convergence de $\left(T\left(\boldsymbol{x}_{0}+\lambda \boldsymbol{x}, \boldsymbol{y}\right), \chi(\boldsymbol{x}, \boldsymbol{y})\right)$ est uniforme dans l'ensemble $\chi \in \mathcal{D}_{(-1,1)^{m} \times E}$, $\left|D_{\boldsymbol{x}}^{\boldsymbol{p}} D_{\boldsymbol{y}}^{\boldsymbol{q}} \chi\right| \leqslant 1$ pour $(\boldsymbol{p}, \boldsymbol{q}) \in \mathfrak{\Xi}$.

Si $\mathfrak{P}$ est l'ensemble $|\boldsymbol{p}| \leqslant k$, nous dirons que la valeur est d'ordre $\leqslant k$.

Si $\mathfrak{S}$ est l'ensemble $|\boldsymbol{p}| \leqslant k,|\boldsymbol{q}| \leqslant l$, nous dirons que la fixation est d'ordre $\leqslant(k, l)$.

Evidemment la valeur est toujours d'ordre fini, et pour tout compact $E \subset \Omega$ il existe un couple $(k, l)$ tel que la fixation soit d'ordre $\leqslant(k, l)$ $\operatorname{sur} E$.

La proposition 1 du No8.1 entraîne la

Proposition 1. Si la valeur est d'ordre C $\mathfrak{P}$, elle est aussi d'ordre $\subset \mathfrak{P}_{0}$, pourvu que $\mathfrak{P}+\frac{1}{2} 1 \subset C\left(\mathfrak{P}_{0}\right)$. Il en est de même pour la fixation.

On voit qu'il n'y a aucune relation entre l'ordre de la fixation et celui de la section. Pourtant, si la fixation sur $E$ et la distribution $T$. dans un ouvert contenant $\left\{\boldsymbol{x}_{0}\right\} \times E$ sont d'ordre $\subset \mathcal{G}$, alors la section $S(\boldsymbol{y})=T\left(\boldsymbol{x}_{0}, \boldsymbol{y}\right)$ est d'ordre $C \mathfrak{Q}$ dans $E$, où $\mathfrak{Q}$ est l'ensemble des $\boldsymbol{q}$ tels que $(\boldsymbol{p}, \boldsymbol{q}) \in \mathfrak{S}$ pour un $\boldsymbol{p}$.

Supposons que la fixation soit d'ordre $C \mathcal{S}$ et que la section soit d'ordre $\subset \mathfrak{Q}$ sur $E$. Il en résulte que $(T, \chi) \rightarrow(S, \psi)$ lorsque

$\left\{\begin{array}{l}\lambda \rightarrow 0, \chi \in \mathcal{D}_{E_{\times} P_{\lambda}\left(\boldsymbol{x}_{0}\right)}, \sup \left|\mathrm{D}_{\boldsymbol{x}}^{\boldsymbol{p}} \mathrm{D}_{\boldsymbol{y}}^{\boldsymbol{\alpha}} \chi\right|=O\left(\frac{1}{\lambda^{|\boldsymbol{p}|+m}}\right) \text { pour }(\boldsymbol{p}, \boldsymbol{q}) \epsilon \mathfrak{G} ; \\ \left.\int D^{\boldsymbol{q}} \chi(\boldsymbol{x}, \boldsymbol{y}) d x \rightarrow D^{\boldsymbol{\alpha}} \psi(\boldsymbol{y}) \text { uniformément (par rapport à } \boldsymbol{y}\right), \\ \text { pour } \boldsymbol{q} \epsilon \Omega .\end{array}\right.$

En particulier, si la fixation est d'ordre $\subset \mathfrak{P} \times \mathcal{Q}$ sur $\Omega$ alors

(8.2.2) $\left\{\begin{array}{r}(T, \alpha(x) \psi(y)) \rightarrow(S, \psi(y)) \\ \text { lorsque } \lambda \rightarrow 0, \alpha \in \mathscr{D}_{P_{\lambda}\left(x_{0}\right)}, \quad \int \alpha d x=1, \sup \left|D^{\boldsymbol{p}} \alpha\right|=O\left(\frac{1}{\lambda^{|\boldsymbol{p}|+m}}\right) \\ \quad \text { pour } \boldsymbol{p} \in \mathfrak{P} ; \\ \text { uniformément dans l'ensemble: } \psi \in \mathscr{D}_{\Omega},\left|D^{\boldsymbol{\alpha}} \psi\right| \leqslant 1 \text { pour } \boldsymbol{q} \in \mathfrak{Q} .\end{array}\right.$

ss) Si l'on remplace $(-1,1)^{m}$ par un ouvert borné (et contenant 0) quelconque, on obtient une condition équivalente. 
Réciproquement on a le

THÉORÈME. Si une distribution $T$ satisfait à la condition (8.2.2), où $\Omega$ est un ouvert de $\left(\mathcal{E}^{n}\right)_{y}$, et si $\mathfrak{P}+\frac{1}{2} \mathbf{1} \subset C\left(\mathfrak{P}_{0}\right), \mathfrak{Q}+\frac{1}{2} \mathbf{1} \subset C\left(\mathfrak{Q}_{0}\right)$, alors on peut fixer $\boldsymbol{x}=\boldsymbol{x}_{0}$ sur $\Omega$ et la fixation est d'ordre $\subset \mathfrak{P}_{0} \times \mathfrak{Q}_{0}$ localement sur $\Omega$.

Démonstration. On peut admettre $S=0$. Selon (8.2.2) nous avons $\left|\left(T\left(\boldsymbol{x}_{0}+\lambda \boldsymbol{x}, \boldsymbol{y}\right), \varphi(\boldsymbol{x}) \psi(\boldsymbol{y})\right)\right| \leqslant \varepsilon_{\lambda}$, où $\lim \varepsilon(\lambda)=0$, lorsque $\varphi \epsilon \mathcal{D}_{(-1,1)^{m}}$, $\left|D^{\boldsymbol{p}} \varphi\right| \leqslant 1$ pour $\boldsymbol{p} \in \mathfrak{P}, \psi \in \mathcal{D}_{\Omega},\left|D^{\boldsymbol{\alpha}} \psi\right| \leqslant 1$ pour $\boldsymbol{q} \in \mathcal{O}$, d'où résulte la conclüsion, en vertu du corollaire $2 \mathrm{du} \mathrm{N}^{0} 8.1$.

Soit $\mathfrak{P}$ l'ensemble $|\boldsymbol{p}| \leqslant k$ et $\mathfrak{P}_{0}$ l'ensemble $|\boldsymbol{p}| \leqslant k_{0}$. On vérifie qu'on a $\mathfrak{P}+1 \frac{1}{2} \subset C\left(\mathfrak{P}_{0}\right)$, pourvu que $\left.k_{0}>k+m / 2^{34}\right)$. On a donc le

CoRolLAIRE. Si $\left(T\left(\boldsymbol{x}_{0}+\lambda \boldsymbol{x}, \boldsymbol{y}\right), \varphi(\boldsymbol{x}) \psi(\boldsymbol{y})\right) \rightarrow(S, \psi)$ lorsque $\lambda \rightarrow 0+$ uniformément pour $\varphi \in \mathcal{D}_{(-1,1)}{ }^{m}, \int \varphi d \boldsymbol{x}=1, \quad \psi \in \mathcal{D}_{\Omega},\|\varphi\|_{k} \leqslant 1,\|\psi\|_{l} \leqslant 1$, et si $k_{0}>k+m / 2, l_{0}>l+n / 2$, alors on peut fixer $\boldsymbol{x}=\boldsymbol{x}_{0}$ sur $\Omega$ et la fixation est d'ordre $\leqslant\left(k_{0}, l_{0}\right)$ localement sur $\Omega$.

Nous complétons maintenant la démonstration du théorème du $\mathrm{N}^{0} 4.5$. $L a$ condition (4.5.3) est suffisante. Soit $E$ un compact contenu dans $\Omega$. D'après le corollaire il suffit de prouver que la convergence de $\left(T_{\lambda}, \varphi(\boldsymbol{x}) \psi(\boldsymbol{y})\right)$ où $T_{\lambda}(\boldsymbol{x}, \boldsymbol{y})=T\left(\boldsymbol{x}_{0}+\lambda \boldsymbol{x}, \boldsymbol{y}\right)$ est uniforme dans un ensemble de la forme $\varphi_{\epsilon} \mathcal{D}_{[-1,1]}, \psi \epsilon \mathcal{D}_{E},\|\varphi\|_{k} \leqslant 1,\|\psi\|_{l} \leqslant 1$. Considérons les fonctionnelles bilinéaires $B_{\lambda}(\varphi, \psi)=\left(T_{\lambda}, \varphi(\boldsymbol{x}) \psi(\boldsymbol{y})\right)$; elles sont définies pour $0<\lambda<\lambda_{0}$ dans l'espace complet $\mathcal{D}_{[-1,1]} \times \mathcal{D}_{\text {II }}$ et elles convergent simplement pour $\lambda \rightarrow 0+$, donc (théorème de Baire) elles sont bornées uniformément dans une boule. On en conclut l'existence de $k, l, M$ tels que $\left|B_{2}(\varphi, \psi)\right| \leqslant M$ pour $\|\varphi\|_{k} \leqslant 1,\|\psi\|_{l} \leqslant 1$; par conséquent les $B_{\lambda}$ sont équicontinues dans le sous-espace $\mathcal{D}_{\left[-1,1 m^{m} \times \mathcal{D}_{E}\right.}$ de l'espace $\mathcal{D}_{[-1,1]}^{k} \times \mathcal{D}_{E}^{l}$ séparable, dans leque l'ensemble $\|\varphi\|_{k+1} \leqslant 1,\|\psi\|_{k_{+1}} \leqslant 1$ est relativement compact. Il en résulte que la convergence de $B_{\lambda}$ est uniforme dans cet ensemble, c. q. f. d. $\left.{ }^{35}\right)$. Soit $\Omega$ un ouvert de $\left(E^{n}\right)_{y}$.

Proposition 2. Si pour tout $\varphi \in(\mathcal{D})_{x}$ tel que $\int p d x=1$ on a

$$
\lim _{\lambda \rightarrow 0+}\left(T, \frac{1}{\lambda^{m}} \varphi\left(\frac{\boldsymbol{x}-\boldsymbol{x}_{0}}{\lambda}\right) \psi(\boldsymbol{y})\right)=(S, \psi)
$$

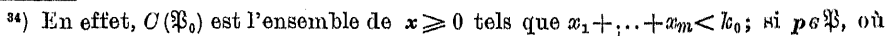
$|\boldsymbol{p}| \leqslant k$, on a $\left(p_{1}+\frac{1}{2}\right)+\cdots+\left(p_{m}+\frac{1}{2}\right) \leqslant k+\frac{1}{2} m<k_{0}$, donc $\boldsymbol{p}+\frac{1}{2} \mathbf{1} 60\left(\Re_{0}\right)$.

${ }^{35}$ ) Ce raisonnement et la proposition 2 du $\mathrm{N}^{0} 8.1$ donnent lo théorème $\mathrm{a}$, une suite $T_{v}$ (ou un filtre de base dénombrable) de distributions converge lorsque $\left(T_{v}, \varphi_{1}\left(x_{1}\right) \ldots p_{m}\left(x_{m}\right)\right)$ converge (simplement); une famille $\left\{T_{t}\right\}$ de distributions est bornée si $\left(T_{1}, p_{1}\left(x_{1}\right) \ldots p_{m}\left(x_{m}\right)\right)$

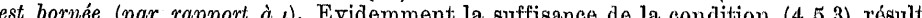
$\theta$ ce théorème qui est une conséquence du théorème des noyaux de L. Schwartz [8] et d'une généralisation du théorème de Banach sur l'opération inverse, due à G Köthe [3]. Notons que réciproquement, le théorème de L. Schwartz résulte faoilement de ce théorème. uniformément dains l'ensemble $\psi \epsilon \mathcal{D}_{\Omega},\|\psi\|_{l} \leqslant 1$ et si $\bar{l}>l+n / 2+1$, alors on peut fixer $\boldsymbol{x}=\boldsymbol{x}_{0}$ sur $\Omega$ et il existe un $k$ tel que la fixation soit d'ordre $\leqslant(k, \bar{l})$ localement sur $\Omega$.

Démonstration. On peut admettre $S=0$. Posons $T_{\lambda}(\boldsymbol{x}, \boldsymbol{y})=$ $=T\left(\boldsymbol{x}_{0}+\lambda \boldsymbol{x}, \boldsymbol{y}\right)$. On a $\lim _{\lambda \rightarrow 0+}\left(T_{\lambda}, \varphi(\boldsymbol{x}) \psi(\boldsymbol{y})\right)=0$ pour tout $\varphi \in(\mathcal{D})_{\boldsymbol{x}}$ uniformément dans l'ensemble $\mathscr{A} \subset \mathcal{D}_{\Omega}$ des $\psi$ tels que $\|\psi\|_{l} \leqslant 1$. Considérons la famille des distributions $U_{\lambda, p}=\left(T_{\lambda}, \psi_{y}\right)$; pour tout $\varphi \in \mathscr{D}_{[-1,1} m$ il existe un $\lambda^{\prime}$ tel que $\left|\left(U_{\lambda, \psi}, \varphi\right)\right| \leqslant 1$ pour $0<\lambda<\lambda^{\prime}$ et $\psi \in \mathscr{A}$, donc (théorème de Baire) il existe un $\lambda_{0}$ et une boule 93 de $\mathscr{D}_{[-1,1]} m$ tel que $\left|\left(U_{\lambda, y}, \varphi\right)\right| \leqslant 1$ pour $0<\lambda<\lambda_{0}, \psi \in \mathscr{S}, \varphi \in 73$. On en conclut l'existence de $k, M$ tels que $\left|\left(T_{\lambda}, \varphi(\boldsymbol{x}) \psi(\boldsymbol{y})\right)\right| \leqslant M$, lorsque $\varphi \in \mathscr{D}_{[-1,1]} m, \psi \in \mathscr{D}_{\Omega},\|\varphi\|_{k} \leqslant 1,\|\psi\|_{l} \leqslant 1$. Soit $\boldsymbol{E}$ un compact contenu dans $\Omega$ et soit $\Delta$ un ouvert borné tel que $B \subset \Delta$, $\bar{\Delta} \subset \Omega$; il en résulte, comme dans la démonstration précédente, que la convergence $\left(T_{\lambda}, \varphi(\boldsymbol{x}) \psi(\boldsymbol{y})\right) \rightarrow 0$ est uniforme dans l'ensemble $\varphi \in \mathcal{D}_{[-1,1]} m$, $\psi \epsilon \mathcal{D}_{\bar{A}},\|\varphi\|_{t_{+1}} \leqslant 1,\|\psi\|_{l_{+1}} \leqslant 1$, donc, d'après le corollaire, la fixation est d'ordre $\leqslant\left(k_{0}, \bar{l}\right)$ sur $E$, où $k_{0}>k+m / 2+1$.

Un raisonnement exactement pareil donne la

Propostrion 3. Si pour tout $\psi \in \mathscr{D}_{\Omega}$ la distribution $U_{v}=(T, \psi)_{y}$ possède au point $\boldsymbol{x}_{0}$ une valeur d'ordre $\leqslant k$ (c'est-à-dire $(T, \alpha(\boldsymbol{x}) \psi(\boldsymbol{y})) \rightarrow$ $\rightarrow(S, \psi)$ lorsque $\cdot \lambda \rightarrow 0, a \in \mathcal{D}_{P_{\mathbf{\alpha}}\left(x_{0}\right)}, \int a d x=1$ et $\left.\|\alpha\|_{k}=O\left(1 / \lambda^{k+m}\right)\right)$ et si $\bar{k}>k+m / 2+1$, alors on peut fixer $\boldsymbol{x}=\boldsymbol{x}_{0}$ sur $\Omega$ et il existe un $l$ tel que la fixation soit d'ordre $\leqslant(\bar{k}, l)$ localement sur $\Omega$.

\subsection{Condition nécessaire et suffisante avec primitive pour l'ordre.} Nous arons le

THÉORÈME 1. Pour que la section $T\left(\boldsymbol{x}_{0}, \boldsymbol{y}\right)=0$ existe sur un ouvert $\Omega \subset\left(\mathcal{E}^{n}\right)_{y}$ et que la fixation soit d'ordre $\subset \odot$ localement sur $\Omega$, it faut et il suffit que pour tout intervalle ouvert $Q$ tel que $\bar{Q} \subset \Omega$ il existe un $\lambda_{0}>0$ tel que dans $P_{\lambda_{0}}\left(\boldsymbol{x}_{0}\right) \times Q$ la distribution $T$ soit de la forme

$$
T=\sum_{(\boldsymbol{p}, \boldsymbol{q}) \in \mathbb{E}} D_{\boldsymbol{x}}^{\boldsymbol{p}} D_{\boldsymbol{y}}^{\boldsymbol{q}} \sigma_{\boldsymbol{p}, \boldsymbol{q}},
$$

où $\sigma_{\boldsymbol{p}, \boldsymbol{\alpha}}$ sont des mesures telles que $\left|\sigma_{\boldsymbol{p}, \boldsymbol{q}}\right|\left(P_{\lambda}\left(\boldsymbol{x}_{0}\right) \times Q\right)=o\left(\lambda^{|\boldsymbol{p}|+m}\right)$.

Démonstration. On vérifie facilement que la condition est suffisante. Nous allons démontrer qu'elle est nécessaire $\left.{ }^{36}\right)$. On peut admettre $\boldsymbol{x}_{0}=0$. Soit $Q$ un intervalle ouvert tel que $\bar{Q} \subset \Omega$; il existe un intervalle

${ }^{\text {se) }}$ Comme dans le $\mathrm{N}^{0} 4.1$ il suffit ici de supposer que $\left(T\left(x_{0}+\lambda_{p} \boldsymbol{x}, \boldsymbol{y}\right), \chi(\boldsymbol{x}, \boldsymbol{y})\right) \rightarrow 0$ uniformément dans l'ensemble $\left.\chi \in \mathcal{D}_{p_{1}\left(\boldsymbol{x}_{0}\right)} \times Q, \mid D_{\boldsymbol{x}}^{\boldsymbol{p}} D_{\boldsymbol{y}}^{\boldsymbol{q}} \chi\right) \mid \leqslant 1$ pour $(\boldsymbol{p}, \boldsymbol{q}) \in \widetilde{S}$, où $\left\{\lambda_{v}\right\}$ est une suite qui satisfait à la condition (4.1.1). 
ouvert $Q_{1}$ tel que $\bar{Q} \subset Q_{1}$ et $\bar{Q}_{1} \subset \Omega$. Posons

$$
\lambda_{v}=\lambda_{0} / 2^{p}, \quad P_{v}=P_{\lambda_{\nu}}(0) \quad(v=0,1, \ldots) ;
$$

si $\lambda_{0}$ a été choisi suffisament petit, on a $\left|\left(T\left(\lambda_{\nu} \boldsymbol{x}, \boldsymbol{y}\right), \chi(\boldsymbol{x}, \boldsymbol{y})\right)\right| \leqslant \varepsilon_{\nu}$ lorsque $\chi \in \mathscr{D}_{P_{1}(0) \times Q_{1}}$ et $\left|D_{\boldsymbol{x}}^{p} D_{\boldsymbol{q}}^{y} \chi\right| \leqslant 1$ pour $(\boldsymbol{p}, \boldsymbol{q}) \in \circlearrowleft(\nu=0,1, \ldots)$, où

$$
\varepsilon_{\nu} \rightarrow 0 \quad \text { en décroissant. }
$$

Nous procédons maintenant selon une idée de L. Schwartz ([7], tome I,p.91-92). Soit $T$ l'espace des systèmes $\left\{\psi_{\boldsymbol{p} \boldsymbol{q}}\right\}_{(\boldsymbol{p}, \boldsymbol{\alpha}) \Theta \mathscr{E}}$, où $\psi_{\boldsymbol{p}, \boldsymbol{\alpha}} \in \mathcal{D}_{P_{1}(0) \times Q_{1}}^{0}$, avec la norme max $\sup \left|\psi_{\boldsymbol{p} \boldsymbol{\alpha}}\right|$; soit $\Delta$ le sous-espace des systèmes de la forme

$\psi_{p q}=D_{x}^{p} D_{y}^{\alpha}, \chi$ où $\chi \in D_{P_{1}(0) \times Q_{1}}$. La fonctionnelle $\xi_{v}$ est bien définie dans $\triangle$ par la formule $\xi_{\nu}\left[\left\{D_{\boldsymbol{x}}^{\boldsymbol{p}} D_{\boldsymbol{y}}^{\boldsymbol{q}} \chi\right\}\right]=\left(T\left(\lambda_{\nu} \boldsymbol{x}, \boldsymbol{y}\right), \chi(\boldsymbol{x}, \boldsymbol{y})\right)$ et sa norme ne surpasse pas $\varepsilon_{v}$; d'après le théorème de Banach-Hahn elle peut être prolongée sur $\Gamma$ de facon que sa norme soit conservée. Le prolongement $\bar{\xi}_{v}$, étant une fonctionnelle linéaire dans $\Gamma$, est de la forme (théorème de Riesz) $\bar{\xi}_{\nu}\left[\left\{\psi_{p \boldsymbol{q}}\right\}\right]=\sum_{\gamma} \int \psi_{\boldsymbol{p} \boldsymbol{q}} d \mu_{\boldsymbol{p} \boldsymbol{q}}^{\boldsymbol{v}}$, où $\mu_{\boldsymbol{p q}}^{\nu}$ sont des mesures telles que $\sum_{\gamma}\left|\mu_{\boldsymbol{p q}}^{\nu}\right|\left(P_{1}(0) \times Q_{1}\right)$ $\leqslant \varepsilon_{v}$. Il en résulte que $T\left(\lambda_{p} \boldsymbol{x}, \boldsymbol{y}\right)=\sum D_{\boldsymbol{x}}^{\boldsymbol{p}} D_{\boldsymbol{y}}^{q} \mu_{\boldsymbol{p} \boldsymbol{q}}^{\nu}$ dans $\stackrel{\gamma}{P_{1}}(0) \times Q_{1}$.

Posons maintenant $\sigma_{p \boldsymbol{q}}^{v}(\boldsymbol{x}, \boldsymbol{y})=\lambda_{v}^{\nu} \mu_{\boldsymbol{p q}}^{\nu}\left(\boldsymbol{x} / \lambda_{p}, \boldsymbol{y}\right)$ dans $P_{\nu} \times Q_{1}$. D'après ce qui précède, on a alors

$$
\begin{aligned}
& T=\sum_{\mathscr{夭}} D_{x}^{p} D_{y}^{\alpha} \sigma_{v p q} \text { dans } P_{\nu} \times Q_{1} \\
& \text { et } \left.\quad\left|\sigma_{\boldsymbol{p q}}{ }_{\boldsymbol{q}}\right| P_{\nu} \times Q_{1}\right) \leqslant \varepsilon_{\nu} \lambda_{p}^{|\boldsymbol{p}|+m} \\
& (\nu=0,1, \ldots ;(\boldsymbol{p}, \boldsymbol{q}) \in \mathcal{S}) .
\end{aligned}
$$

Posons $\varrho_{p q}^{\nu}=\sigma_{p q}^{p}-\sigma_{p q}^{\nu+1}$ dans $P_{p} \times Q_{1}$; on a donc

$$
\sum_{\nu} D_{\boldsymbol{x}}^{\boldsymbol{p}} D_{\boldsymbol{y}}^{\boldsymbol{q}} \varrho_{\boldsymbol{p} \boldsymbol{\alpha}}^{\nu}=0 \text { dans } P_{\nu} \times Q_{1} \text { et }\left|\varrho_{\boldsymbol{p q}}^{\nu}\right|\left|\left(P_{\nu} \times Q_{1}\right)\right| \leqslant 2 \varepsilon_{\nu-1} \lambda_{\nu-1}^{\lambda \boldsymbol{p} \mid+\cdots} .
$$

Selon le lemme $4 \mathrm{du} \mathbb{N}^{0} 2.5$ le système de mesures $\varrho_{p \alpha}^{v}$ possède un prolon-

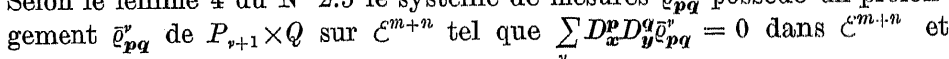
(en tenant compte de (8.3.4))

$$
\begin{aligned}
\left|\bar{\varrho}_{\boldsymbol{p q}}^{\prime \prime}\right|\left(P_{t}(0) \times Q\right) & \leqslant 2 K \varepsilon_{\nu-1} \lambda_{p-1}^{|\boldsymbol{p}|+m}\left(1+\left(\frac{t}{\lambda_{p+1}}\right)^{|\boldsymbol{p}|}\right) \\
& =2 \cdot 4^{|\boldsymbol{p}|+m} K \varepsilon_{p-1} \lambda_{p+1}^{m}\left(\lambda_{p+1}^{|\boldsymbol{p}|}+t^{|\boldsymbol{p}|}\right)
\end{aligned}
$$

où $K$ ne dépend que de $\subseteq, Q, Q_{1}$. En posant $\bar{\sigma}_{p q}^{p}=\sigma_{p q}^{0}+\bar{\varrho}_{\boldsymbol{p} \alpha}^{1}+\ldots+\bar{\varrho}_{\boldsymbol{p} \chi}^{p}$ dans $P_{0} \times Q_{1}$, on a donc $\bar{\sigma}_{p q}^{p}=\sigma_{p q}^{v}$ dans $P_{v+1} \times Q$ et (d'après (8.3.4))

(8.3.6) $\quad T=\sum_{\mathscr{S}} D_{\boldsymbol{x}}^{\boldsymbol{p}} D_{\boldsymbol{y}}^{\boldsymbol{q}} \bar{\sigma}_{\boldsymbol{p} \boldsymbol{\alpha}}^{\nu}$ dans $P_{0} \times Q_{1} \quad$ et $\quad\left|\bar{\sigma}_{\boldsymbol{p q}}^{\nu}\right|\left(P_{p+1} \times Q\right) \leqslant \varepsilon_{\nu} \lambda_{\nu}^{|\boldsymbol{p}|+m}$.
L'inégalité (8.5.3) donne (en tenant compte de (8.3.2)) la majoration suivante:

$$
\left|\bar{\sigma}_{\boldsymbol{p \alpha}}^{p+x}-\bar{\sigma}_{\boldsymbol{p q}}^{p}\right|\left(P_{t}(0) \times Q\right) \leqslant K_{1} \varepsilon_{\nu} \lambda_{p}^{m}\left(\lambda_{p}^{|\boldsymbol{p}|}+t^{|\boldsymbol{p}|}\right) \quad \text { lorsque } \quad t \leqslant \lambda_{0},
$$

où $K_{1}$ ne dépend que de $\mathfrak{S}, Q, Q_{1} . \Pi$ en résulte que pour tout $(\boldsymbol{p}, \boldsymbol{q}) \in \mathfrak{S}$ la suite $\left\{\sigma_{\boldsymbol{p} \boldsymbol{\alpha}}^{\prime \prime}\right\}$ converge vers une mesure $\sigma_{\boldsymbol{p} \boldsymbol{q}}^{\nu}$ dans $P_{0} \times Q$ (pour $\left.\nu \rightarrow \infty\right)$ et on a, en vertu de (8.3.6),

$$
T=\sum_{\gamma} D_{\boldsymbol{x}}^{\boldsymbol{p}} D_{\boldsymbol{y}}^{\boldsymbol{q}} \sigma_{\boldsymbol{p} \boldsymbol{q}} \text { dans } P_{0} \times Q \quad \text { et } \quad\left|\sigma_{\boldsymbol{p q} \boldsymbol{q}}\right|\left(P_{\boldsymbol{v}+1} \times Q\right) \leqslant\left(1+2 K_{1}\right) \varepsilon_{\nu} \lambda_{\boldsymbol{p}}^{|\boldsymbol{p}|+m} \text {. }
$$

On en conclut facilement que $\left|\sigma_{\boldsymbol{p q}}\right|\left(P_{\lambda}(0) \times Q\right)=o\left(\lambda^{|\boldsymbol{p}|+m}\right)$, ce qui termine la démonstration.

Le théorème 1 montre que pour que la fixation $\boldsymbol{x}=\boldsymbol{x}_{0}$ soit localement, d'ordre $\subset \subseteq$, , il faut et il suffit que la distribution soit (localement, dans un voisinage de l'hyperplan $\boldsymbol{x}=\boldsymbol{x}_{0}$ ) de la forme

$$
T(\boldsymbol{x}, \boldsymbol{y})=S(\boldsymbol{y})+\sum_{\mathscr{E}} D_{\boldsymbol{x}}^{\boldsymbol{p}} D_{\boldsymbol{y}}^{\boldsymbol{q}} \sigma_{\boldsymbol{q} \boldsymbol{p}},
$$

où $\sigma_{\boldsymbol{p q}}$ sont des mesures telles que $\left|\sigma_{\boldsymbol{p q}}\right|\left(P_{\lambda}\left(\boldsymbol{x}_{0}\right) \times Q\right)=o\left(\lambda^{|\boldsymbol{p}|+m}\right)$.

Soit $\mathfrak{Q}$ l'ensemble des $\boldsymbol{q}$ tels que $(\boldsymbol{p}, \boldsymbol{q}) \in \mathcal{S}$ pour un $\boldsymbol{p}$ et supposons que la fixation soit localement d'ordre $\mathcal{C} \mathfrak{E}$. On voit donc que la condition que $T$ soit d'ordre $C \mathcal{S}$ localement dans un voisinage de l'hyperplan $\boldsymbol{x}=\boldsymbol{x}_{0}$ équivaut à celle que $S$ soit localement d'ordre $C \mathfrak{Q}$. Selon un théorème de L. Schwartz ([7], tome I, p. 91-92) on a alors un développement (local) $S=\sum_{\Omega} \mathrm{D}^{\alpha} \varrho_{\alpha}$, où $\varrho_{\boldsymbol{\alpha}}$ sont des mesures, et par suite

$$
S=\sum_{\gamma} D_{\boldsymbol{x}}^{\boldsymbol{p}} D_{\mathbf{y}}^{\underline{q}} \frac{\left(\boldsymbol{x}-\boldsymbol{x}_{0}\right)^{p}}{\boldsymbol{p} !} \varrho_{\boldsymbol{p q}},
$$

où $\varrho_{\boldsymbol{p}}=\varrho_{\boldsymbol{q}}$ pour un $\boldsymbol{p}$ tel que $(\boldsymbol{p}, \boldsymbol{q}) \epsilon \gamma$ et $\varrho_{\boldsymbol{p} q}=0$ pour les autres $\boldsymbol{p}$. Nous avons donc l'énoncé suivant:

Pour que la distribution $T$ et la fixation $\boldsymbol{x}=\boldsymbol{x}_{0}$ dans $T$ soient localement d'ordre C $\mathcal{~ S , ~ i l ~ f a u t ~ e t ~ i l ~ s u f f i t ~ q u e ~} T$ soit localement de la forme

$$
T(\boldsymbol{x}, \boldsymbol{y})=\sum_{\gamma} D_{\boldsymbol{x}}^{\boldsymbol{p}} D_{\boldsymbol{y}}^{\boldsymbol{q}} \mu_{\boldsymbol{p q}},
$$

où

$$
\begin{aligned}
\mu_{\boldsymbol{p q}}(\boldsymbol{x}, \boldsymbol{y}) & =\frac{\left(\boldsymbol{x}-\boldsymbol{x}_{0}\right)^{\boldsymbol{p}}}{\boldsymbol{p} !} \varrho_{\boldsymbol{p q}}(\boldsymbol{y})+\sigma_{\boldsymbol{p q}}(\boldsymbol{x}, \boldsymbol{y}), \\
\left|\sigma_{\boldsymbol{p q}}\right|\left(P_{\lambda}\left(\boldsymbol{x}_{0}\right) \times Q\right) & =o\left(\lambda^{|\boldsymbol{p}|+m}\right) \quad\left(\sigma_{\boldsymbol{p q}}, \varrho_{\boldsymbol{p q}} \text { étant de mesures }\right) .
\end{aligned}
$$


Dans le cas de la valeur nous avons, en particulier:

THÉORÈme 2. Pour que la valeur $T\left(\boldsymbol{x}_{0}\right)$ existe et soit d'ordre $\subset \mathfrak{P}$, il faut et il suffit que dans une voisinage de $\boldsymbol{x}_{0}$ la distribution $T$ soit de la forme

(8.3.9) $\quad T=T\left(x_{0}\right)+\sum_{\mathfrak{*}} D^{\boldsymbol{p}_{\sigma_{\boldsymbol{p}}}}, \quad$ ò̀ $\quad\left|\sigma_{\boldsymbol{p}}\right|\left(P_{\lambda}\left(\boldsymbol{x}_{0}\right)\right)=o\left(\lambda^{|\boldsymbol{p}|+m}\right)$

( $\sigma_{\boldsymbol{p}}$ étant des mesures).

Finalement les théorèmes 1 et 2 donnent les développements

(8.3.10) $(T(\boldsymbol{x}, \boldsymbol{y}), \chi(\boldsymbol{x}, \boldsymbol{y}))=\left(S, \int \chi(\boldsymbol{x}, \boldsymbol{y}) d \boldsymbol{x}\right)_{y}+\sum_{\odot} \int D_{x}^{\boldsymbol{p}} D_{\boldsymbol{y}}^{\boldsymbol{g}} \chi(\boldsymbol{x}, \boldsymbol{y}) d \sigma_{\boldsymbol{p} \boldsymbol{q}}$,

où $\left|\sigma_{p \boldsymbol{q}}\right|\left(P_{\lambda}\left(\boldsymbol{x}_{0}\right) \times Q\right)=o\left(\lambda^{|\boldsymbol{p}|+m}\right)$, pour $\chi \boldsymbol{\epsilon}^{\left(D_{\mathcal{C}} m_{\times Q}\right.}$, si $S(\boldsymbol{y})=T\left(\boldsymbol{x}_{0}, y\right)$ et si la fixation est d'ordre $C \subseteq$ sur un ouvert contenant $\bar{Q}$, et

$$
\begin{aligned}
(T(\boldsymbol{x}), \varphi(\boldsymbol{x})) & =T\left(\boldsymbol{x}_{0}\right) \int \varphi(\boldsymbol{x}) d \boldsymbol{x}+\sum_{*} \int D^{\boldsymbol{p}} \varphi d \sigma_{\boldsymbol{p}}, \\
\left|\sigma_{\boldsymbol{p}}\right|\left(P_{\lambda}\left(\boldsymbol{x}_{0}\right)\right) & =o\left(\lambda^{|\boldsymbol{p}|+m}\right),
\end{aligned}
$$

si la valeur est d'ordre $C \mathfrak{P}$.

\section{Travaux cités}

[1] A. Denjoy, Sur l'intégration des coefficients différentiels d'ordre supérieur, Fund. Math. 25 (1935), p. 237-320.

[2] H. König, Neue Begründung der Theorie der ,Distributionen” von L. Solhwartz, Math. Nachrichten 9 (1953), p. 129-148.

[3] G. Köthe, Über zwei Sätze von Banach, Math. Zeitsehr. 53 (1950), p. $203-209$.

[4] S. Łojasiewicz, Sur la valeur d'une distribution dans un point, Bull. Ao. Polon. Sci. Cl. III, 4 (1956), p. $239-242$.

[5] - Sur la valeur et la limite d'une distribution dans un point, Studia Math. $16(1957), p .1-36$

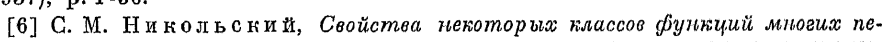
ременных на дифференцируемых миогообразиях, Мат. сборнит 33 (75) (1953), p. $261-326$.

[7] L. Schwartz, Théorie des distrilntions, $I, I I$, l'aris 1950 at 1951

[8] - Théorie des noyaux, Proc. Int. Congress of Math. I, Cambridge, Massachusetts 1950 , p. $220-230$.

[9] Z. Zieleźny, Sur 7a définition de Lojasiewicz de la valeur diune distribution dans un point, Bull. Ac. Polon. Sci., Cl. III, 3 (1955), p. 519-520.

Reģu par la Rédaction le 5.12.1956

\section{On certain "weak" properties of vector-valued functions}

\section{A. ALEXIEWICZ (Poznań)}

The starting point of this Note is the following theorem of B. J. Pettis $\left([6]\right.$, p. $\left.\left.257^{1}\right)\right)$ : a vector-valued ${ }^{2}$ ) function from a measure space to a Banach space $X$ is (Bochner) measurable if and only if it is almost separably valued $^{3}$ ) and for every $\gamma$ belonging to a norming set of functionals the function $\gamma x(t)$ is measurable. The subset $\Gamma$ of the space $\Xi$, conjugate to $X$, is called norming if there are two positive constants $A$ and $B$ such that

$$
\sup \{A|\gamma x|: \gamma \in \Gamma,\|\gamma\| \leqslant B\} \geqslant\|x\|
$$

for every $x$. In this Note we prove that the set $\Gamma$ in the above statement may be replaced by any total subset of $\Xi$ (the set $\Gamma$ is total if $\gamma x=0$ for any $\gamma \in \Gamma$, implies $x=0$ ). Every norming set is necessarily total; the converse, however, is not true, as is shown by the following example of Mazurkiewicz [7]. Suppose that the set of all pairs $(i, k)$ of positive integers is arranged into a single sequence, and let $\nu(i, k)$ denote the place occupied there by $(i, k)$. Then in the space $c_{0}$ of the sequences $x=\left\{x_{n}\right\}$, convergent to zero, consider the set of all the functionals

$$
\xi_{i k}(x)=\frac{x_{1}}{2^{1}}+\ldots+\frac{x_{2 i+1}}{2^{2 i+1}}+i x_{2 v(i, k)}
$$

where $i, k=1,2, \ldots$; the linear span $\Gamma$ of this set is linear, total but not norming.

1. Let $X$ be a separable (real or complex) Banach space, let $\Xi$ be the conjugate space, and let $\Gamma$ be a linear subset of $\Xi$. It is well known that the set $\Gamma$ is total if and only if $\bar{\Gamma}$, its closure in the $\sigma(\Xi, X)$ topo-

1) Numbers in brackets refer to the bibliography at the end of this paper

2) In the sequel all Banach-space-valued functions will be called simply vector. -valued. Numerically valued functions will be called functions.

3) i.e., there exists a subset $N$ of measure zero such that the set $\{y: y=x(t)$, $t$ non $6 N\}$ is separable.

Studia Mathematica T. XVII 